\title{
Private and Common Value Auctions with Ambiguity over Correlation
}

\author{
Krittanai Laohakunakorn, Gilat Levy and Ronny Razin, LSE ${ }^{1}$
}

Abstract: We analyse auctions when individuals have ambiguity over the joint information structures generating the valuations and signals of players. We analyze how two standard auction effects interact with the ambiguity of bidders over correlation structures. First, a "competition effect" arises when different beliefs about the correlation between bidders' valuations imply different likelihoods of facing competitive bids. Second, a "winner's value effect" arises when different beliefs imply different inferences about the winner's value. In the private values case, only the first effect exists and this implies that the distribution of bids first order stochastically dominates the distribution of bids in the absence of ambiguity. In common value auctions both effects exist and we show that compared to the canonical model, both in the first-price and second-price auctions, these effects combine to imply that the seller's revenue decreases with ambiguity (in contrast with the private values case). We then characterise the optimal auction in both the private and common value cases. A novel feature that arises in the optimal mechanism in the common values case is that the seller only partially insures the high type against ambiguity.

\section{Introduction}

In auctions, as in many other strategic situations, individuals often have a good understanding of their own private information but they might know less about others' information sources. For example, in auctions for drilling rights, a company might understand the test results that it conducted but might be worried that these results might be correlated with those of other firms. Similarly the evaluation one gets about a piece of art on sale, might be correlated in complex ways to the evaluations othe bidders might get.

\footnotetext{
${ }^{1}$ This project has received funding from the European Union's Horizon 2020 research and innovation programme under grant agreement No SEC-C413. We thank Subir Bose and Francesco Nava for helpful comments.
} 
In these situations bidders might worry about their lack of understanding of the correlation structure between their own information and that of other bidders. ${ }^{2}$ These considerations are important for their bidding behaviour. In private value auctions, beliefs about correlation influence the assessment of bidders about the competition they might face. In common value auctions, such beliefs also affect the bidders' valuation of the good, which implies an additional effect on the bidders' strategies.

In this paper we analyze private and common value auctions when individuals have ambiguity over the joint information structures generating valuations and signals. Specifically, we assume that individuals know the marginal information structure generating a value or a signal to each bidder, but that they are aware that their information sources might be correlated to a degree, and face ambiguity over the possible correlation scenarios. We propose a simple model to analyze ambiguity over correlation structures that is tailored to the comparison with the standard model. ${ }^{3}$ In particular, we use a single parameter, $a$, to bound the degree of pointwise mutual information of the information structures. ${ }^{4}$ When an individual receives a signal and contemplates what strategy to play, she faces ambiguity aversion (as in Gilboa and Schmeidler 1989) about the set of potential joint information structures, that are bounded by $a$, which govern the valuations and signals of the two bidders.

We analyze a two-bidder, binary-value and binary signal auction (in Section 5 we extend the analysis to models with continuous signals and with many bidders). In this model, the set of information structures that the bidders considers is centered around the true canonical case of independent values and signals, and takes the following form. Consider the two bidders, 1 and 2 , with values $\mathbf{v}=\left(v_{1}, v_{2}\right)$ each receiving a signal, $s_{1}$ and $s_{2}$ respectively. Let $q(\mathbf{s} \mid \mathbf{v})$ denote a joint probability of the signal vector $\mathbf{s}=\left(s_{1}, s_{2}\right)$ conditional on the valuations for the good, and $q_{1}\left(s_{1} \mid \mathbf{v}\right)$ and $q_{2}\left(s_{2} \mid \mathbf{v}\right)$ denote the marginal probabilities of $s_{1}$ and $s_{2}$ conditional on $\mathbf{v}$. Similarly, let $p(\mathbf{v})$ denote the prior over $\mathbf{v}$, with $p_{i}\left(v_{i}\right)$ the marginal prior. We assume that individuals' ambiguity is over the set of joint information structures satisfying $\frac{1}{a} \leq \frac{p(\mathbf{v}) q(\mathbf{s} \mid \mathbf{v})}{\prod_{i=1,2} p_{i}\left(v_{i}\right) \prod_{i=1,2} q_{i}\left(s_{i} \mid \mathbf{v}\right)} \leq a$ for some finite parameter $a \geq 1$, for any $\mathbf{s}, \mathbf{v}{ }^{5}$ In other words, the (exponential) pointwise mutual information (ePMI) is

\footnotetext{
${ }^{2}$ Here we consider sophisticated individuals who entertain the possibility that such correlation might exist. A recent literature looks at naive individuals who are not aware of the correlation between sources of information, i.e., correlation neglect. See Ortoleva and Snowberg (2015), Glaeser and Sunstein (2009) and Levy and Razin (2015a, 2015b), Eyster and Weizsacker (2011), Kallir and Sonsino (2009) and Enke and Zimmermann (2013).

${ }^{3}$ Auctions are typically analyzed under the assumption of conditionally-independent private information, with the bidders aware of this fact.

${ }^{4}$ See Church and Hanks (1991).

${ }^{5}$ Levy and Razin (2018) show that this restriction provides a meaningful way to constrain the set of ambiguous beliefs.
} 
bounded. The higher is $a$, the larger is the ambiguity. When $a=1$ we are back in the standard model with conditionally independent signals and no ambiguity. This framework allows us to analyze ambiguity in the private value auction (where we focus on ambiguity over $p(\mathbf{v})$ ), and in the common value auction (where we focus on ambiguity over $q(\mathbf{s} \mid \mathbf{v})$ ).

In the model, ambiguity over information structures is exogenous but ambiguity over the valuations or the equilibrium bids of opponents is endogenous, and depends on the strategic interaction. An ambiguity averse bidder focuses attention on beliefs that minimise her expected utility. This will be reflected in beliefs that either put weight on more competition, believing the other bidders have similar valuations, or on a low value of the good. The former effect, the "competition effect", is present in both private and common value auctions. The second effect, a "winner's value effect" exists only in common value auctions.

We analyze the equilibria in the second-price and first-price auctions. We first focus on the competition effect. We show how this effect implies overbidding in the private values case; the distribution of bids in the first price auction in the face of ambiguity firstorder stochastically dominates that in the standard model. This implies that ambiguity is beneficial for the seller as her revenue increases with $a$. In contrast, the "winner's value effect" generally implies that bidders should shed their bids. We show that in commonvalue auctions, in which both effects are present, the "winner's value effect" is stronger and the seller's revenue decreases in ambiguity. In particular, the low type always shed their bids while the high type do so unless ambiguity is small and signals too imprecise. In Section 5 we show that this result (and the intuition behind it) also holds when we consider an environment with many bidders, as well as an environment with continuous signals.

We then turn to consider optimal auction design in the face of ambiguity over correlation structures. We first show that the above results about the seller's revenue also hold for the optimal auction; the seller's optimal revenue increases in ambiguity in the private values case but decreases in the common value case. In the private values case our results are consistent with Bose et al (2006) who show that the optimal mechanism fully insures the bidders against ambiguity. In Bose et al (2006) bidders believe that valuations are independently drawn, but face ambiguity regarding the particular distributions of valuations that others have. In contrast, in our model bidders know the marginal distribution of valuations but face ambiguity about the joint distribution of valuations. Still, the full insurance result holds in our setting when values are private.

The full insurance result implies that the competition effect does not arise in equilibrium in the optimal auction. However, there is a sense in which the seller exploits the competition effect to increase her revenue. Since under the worst case belief the high type 
believes that her opponent is likely to also be a high type, she is less willing to deviate than when there is no ambiguity, and the seller is able to extract more rent from the high type. Thus, the seller's revenue increases in ambiguity.

In the common value case, insuring the buyers against ambiguity is not necessarily optimal. In the standard case without ambiguity, any optimal mechanism must allocate the good with probability 1 and fully extract rent. However, any such mechanism cannot be a full insurance mechanism. To prevent the high type from deviating, the seller has to conduct side bets with the low type. Under ambiguity, these side bets are costly since the expected payment to the seller is smaller under the seller's belief than under the worst case belief of the buyer. However, if ambiguity is small or if the signals are very precise, these side bets remain optimal and the low type is not insured against ambiguity.

Furthermore, even though there is no need to conduct side bets with the high type to satisfy any incentive constraint, it is also not necessarily optimal to fully insure the high type. This is because to fully insure the high type, the allocation rule needs to be independent of the type of her opponent. Otherwise, the winner's value effect implies that the high type will underestimate the value of the object. However, when the ambiguity is small or the signals are precise, the seller will allocate the good to the high type with higher probability when her opponent is a low type in order to slacken the incentive constraint. In this case, the seller provides partial insurance by also asking the high type to pay more when her opponent is low type. Under the worst case belief, the high type does not care about the type of her opponent, but undervalues the object conditional on winning. In other words, the seller insures the buyer against the competition effect but not the winner's value effect.

On the other hand if the signals are very imprecise and the ambiguity large, the seller finds it optimal to fully insure the buyers against the ambiguity, so that the allocation of the good does not depend on their signals. As a result, the high type earns positive rents in equilibrium. Finally, we show that the seller's revenue in the optimal mechanism is decreasing in the amount of ambiguity, as we found in both the first and second price auctions.

Our paper is related to a recent literature on ambiguity and auctions. As far as we know, our paper is the first to analyse ambiguity in common-value auctions. For privatevalue auctions, Salo and Weber (1995) and Chen et al (2007) show how ambiguity aversion translates to higher bids as individuals underestimate their winning probabilities. ${ }^{6} \mathrm{We}$ complement their analysis by defining ambiguity differently, and more importantly, by considering the common-values case and the comparison with the private-values case,

\footnotetext{
${ }^{6}$ Chen et al (2007) however provide experimental evidence that bids are lower in the presence of ambiguity in first and second-price auctions with independent private values.
} 
both positively and normatively. Bose et al (2006) analyze optimal auction mechanisms for private-value auctions with ambiguity over other bidders' valuations. They show that the seller will fully insure the buyers against ambiguity. Again, our key contribution is to analyze the common-values case and compare it to the private-values case. Specifically, we show that in the common value auction sometimes only partial insurance arises. Lo (1998) shows that the first-price auction dominates the second-price auction in some environments. He uses a multiple priors approach and shows that equilibrium bids are simply determined as if all players hold the worst-case prior. In our analysis players with different signals focus on different beliefs so the model is not equivalent to one in which players start for example from a mis-specified model with wrong beliefs.

Bose and Renou (2014) study how principals can use ambiguous mechanisms to implement social welfare functions that are not attainable under unambiguous mechanisms. In particular, they construct ambiguous communication mechanisms between the agents and a moderator resulting with agents updating to sets of beliefs. Hanany et al (2018) is a recent contribution to the study of incomplete information games and ambiguity. Finally, Bergemann, Brooks and Morris (2015) consider private values auctions and study the set of achievable utilities when considering, as modelers, the set of different feasible information structures. Our analysis is different as in our approach it is the economic agents, rather than the modeler, who span the possible information structures. In addition, we restrict the set of possible information structures using the notion of pointwise mutual information. We show how this shifts equilibrium behaviour in a non-trivial way.

\section{The Model}

We consider a simple symmetric auction with two bidders (1 and 2), each with two possible valuations $v \in\{L, H\}$ where $0 \leq L<H=1$. For expositional purposes we will focus on the case of $L=0$ in the body of the paper. Results are easily generalised to $L>0$ (see the Appendix where we also provide related results for the case of continuos valuations and signals).

We will consider two cases, private values and common values. In the private value case we will assume that valuations are independent across bidders and distributed uniformly. In the common value case we will assume that the valuations are fully positively correlated with a uniform prior. Bidders know if they are in the private or common values case.

The paper focuses on ambiguity over joint information structures. We model this ambiguity by spanning a set of possible symmetric information structures around the true information structures mentioned above for the private and common value cases. In particular, we assume that bidders know the following aspects of the environment: 
1. The state space. A state is a vector of valuations $\mathbf{v}=\left(v_{1}, v_{2}\right) \in\{L, H\}^{2}$.

2. Priors about the state. Each bidder knows that the marginal prior distributions are uniform; that is, the probability that each bidder has a low value is a half.

3. Signals. Each bidder $i=1,2$ observes a private signal $s_{i} \in\{l, h\}$.

Bidders entertain a set of possible joint information structures that are consistent with the above three aspects of the environment. A joint information structure is given by $\left(\{L, H\}^{2},\{l, h\}^{2}, q(\mathbf{s}, \mathbf{v}), \mathbf{p}(\mathbf{v})\right)$ where $q(\mathbf{s}, \mathbf{v})$ is a joint probability on $\{l, h\}^{2} \times\{L, H\}^{2}$ and $\mathbf{p}(\mathbf{v})$ a distribution over $\{L, H\}^{2}$ that is consistent with uniform marginals.

To define the level of ambiguity, we use a simple one-parameter characterization for the set of joint information structures introduced in Levy and Razin (2018). This characterization uses the exponent of the pointwise mutual information (ePMI) to define bounds on the correlation between information structures. Specifically, for each bidder let $q_{i}\left(s_{i} \mid \mathbf{v}\right)$ denote the marginal conditional probability of receiving the private signal $s_{i} \in\{l, h\}$ given state $\mathbf{v}$ and let $p_{i}\left(v_{i}\right)$ denote the marginal prior. We assume the following:

Assumption A1: There is a parameter $1 \leq a<\infty$, such that each bidder only considers joint information structures, $\left(\{L, H\}^{2},\{l, h\}^{2}, q(\mathbf{s}, \mathbf{v}), p(\mathbf{v})\right)$, so that at any state $\boldsymbol{\omega} \in\{L, H\}^{2}$ and for any vector of signals $\mathbf{s} \in\{l, h\}^{2}$,

$$
\frac{1}{a} \leq \frac{p(\mathbf{v}) q(\mathbf{s} \mid \mathbf{v})}{\prod_{i=1,2} p_{i}\left(v_{i}\right) \prod_{i=1,2} q_{i}\left(s_{i} \mid \mathbf{v}\right)} \leq a .^{7}
$$

The parameter $a$, the PMI-bound, describes the extent of the ambiguity a bidder faces over the set of correlation scenarios. It is straightforward to see that ambiguity is larger when $a$ is larger. The formulation of the set is general in the sense that it is detail-free in terms of the underlying distribution functions. It also captures the maximal set of joint information structures with correlation bounded by $a$. Note that a joint information structure which satisfies independence would have $a=1$ at any point; whenever a joint information structure does not satisfy independence then the ePMI is less than 1 for some $(\mathbf{s}, \mathbf{v})$, and is greater than 1 for some $\left(\mathbf{s}^{\prime}, \mathbf{v}^{\prime}\right){ }^{8}$ A higher $a$ implies that bidders consider information structures that are more concordant; for more on pointwise mutual information as a measure of correlation see Levy and Razin (2018).

In Section 3 we will analyze first-price and second-price auctions for the private values and common values cases, while Section 4 will provide the optimal auction analysis. In all

\footnotetext{
${ }^{7}$ All the results can be easily generalized if instead of the lower bound $\frac{1}{a}$ we use some finite $b<1$.

${ }^{8}$ It is then impossible to consider only priors/information structures with ePMI that is only higher (lower) than 1.
} 
these cases, an equilibrium is denoted by a pair of bidding strategies for the two players, $\left(b^{1}\left(s_{1}\right), b^{2}\left(s_{2}\right)\right)$, and a symmetric equilibrium has $b^{1}()=.b^{2}(.) \equiv b($.$) . We consider max-$ min behaviour. Specifically, in equilibrium, given an observed signal, a bidding strategy maximizes the utility of the individual under the worst-case information structure.

Our framework is flexible to consider different types of underlying correlations, which will be tied to the private/common values environment (indeed in Section 5 we consider environments with continuous signals and with many bidders). To complete the model, we now specify the feasible information structures considered by bidders in these two environments.

\subsection{Private values}

In the private values model bidders know their values (and know they are in the private values model). As signals are fully informative about one's own value, ambiguity will arise about the correlation in the prior distribution $\mathbf{p}(\mathbf{v})$. Specifically, each prior distribution must be consistent with the uniform marginal prior distributions, so that the set of functions $\mathbf{p}(\mathbf{v})$ for $\mathbf{v} \in\{0,1\}$ are represented by:

TABle 1: JOINT INFORMATION STRUCTURES FOR THE PRIVATE VALUE CASE

$\begin{array}{ccc}\mathbf{p}(\mathbf{v}) & 0 & 1 \\ 0 & \alpha & \frac{1}{2}-\alpha \\ 1 & \frac{1}{2}-\alpha & \alpha\end{array}$

Ambiguity is over the parameter $\alpha$ then. Given $a$, the ePMI constraints impose the following restrictions on $\alpha$ :

$$
\frac{1}{a} \leq 4 \alpha \leq a, \frac{1}{a} \leq 4\left(\frac{1}{2}-\alpha\right) \leq a
$$

It is easy to see that the higher is $a$, the larger is the set of possible information structures that are considered by bidders. From the above it is also easy to see that each bidder can consider $\alpha$ satisfying

$$
\frac{1}{4} a=\underline{\alpha}(a) \leq \alpha \leq \bar{\alpha}(a)=\frac{1}{2}\left(1-\frac{1}{2} a\right)
$$

\subsection{Common values}

In the common values case, the ambiguity of the bidders will affect their perception about the correlation between their signals and those of their opponents, through $q(\mathbf{s} \mid \mathbf{v})$. In the previous case we had fixed the marginal priors; here we fix the marginal distributions of the signals. In particular, there are two states of the world, $\mathbf{v} \in\{(1,1),(0,0)\}$. The probability 
of receiving the signal $l$ in state $\mathbf{v}=(0,0)$, or the signal $h$ in state $\mathbf{v}=(1,1)$, is $q>\frac{1}{2}$ i.e., $q(s=l \mid(0,0))=q(s=h \mid(1,1))=q .{ }^{9}$ We assume that the true joint probability distribution, $q\left(s_{1}, s_{2} \mid \mathbf{v}\right)$, satisfies conditional independence, so that $q\left(s_{1}, s_{2} \mid \mathbf{v}\right)=\prod_{i=1,2} q_{i}\left(s_{i} \mid \mathbf{v}\right)$. However, while individuals know the true marginal probability distribution generating both their signals, they have ambiguity over the set of joint information structures.

With the above specification, we can represent the family of information structures the bidder entertain by: ${ }^{10}$

TABle 2: Joint information STRUCTURES FOR THE COMMON VALUE CASE

$\begin{array}{llllll}(0,0) & l & h & (1,1) & l & h \\ l & \alpha_{0} & q-\alpha_{0} & l & \alpha_{1} & 1-q-\alpha_{1} \\ h & q-\alpha_{0} & 1-2 q+\alpha_{0} & h & 1-q-\alpha_{1} & 2 q-1+\alpha_{1}\end{array}$,

Under independence $(a=1), \alpha_{0}=q^{2}$ and $\alpha_{1}=(1-q)^{2}$. In this case then, $\alpha_{0}$ and $\alpha_{1}$ are the parameters over which there is ambiguity, as we define below. It is then easy to derive the bounds for these parameters using the ePMI constraints. Specifically, for a general $a$, the ePMI constraints imply the following bounds on the values of the $\alpha^{\prime} s$ :

$$
\begin{aligned}
& \underline{\alpha}_{0}(a) \leq \alpha_{0} \leq \bar{\alpha}_{0}(a) \\
& \underline{\alpha}_{1}(a) \leq \alpha_{1} \leq \bar{\alpha}_{1}(a)
\end{aligned}
$$

where:

$$
\begin{aligned}
& \underline{\alpha}_{0}(a)=\frac{1}{a}(1-q)^{2}+2 q-1, \underline{\alpha}_{1}(a)=\frac{1}{a}(1-q)^{2} \\
& \bar{\alpha}_{0}(a)=\left\{\begin{array}{ll}
a(1-q)^{2}+2 q-1 & a \leq \frac{q}{1-q} \\
q-\frac{1}{a} q(1-q) & a>\frac{q}{1-q}
\end{array}, \bar{\alpha}_{1}(a)= \begin{cases}a(1-q)^{2} & a \leq \frac{q}{1-q} \\
1-q-\frac{1}{a} q(1-q) & a>\frac{q}{1-q}\end{cases} \right.
\end{aligned}
$$

\section{The competition and winner's value effects}

We now show how ambiguity over correlation can affect bids in two different ways. The competition effect arises as ambiguity will play a role in shaping beliefs about the probable bids of the opponent. The winner's value effect will arise as ambiguity may play a role in shaping beliefs about one's own valuation given the information held by the opponent. Naturally, only the first effect will arise in the private values case while both will arise in the common values case.

\footnotetext{
${ }^{9}$ The analysis can be extended to non-symmetric marginal probability distributions.

${ }^{10}$ The table describes an information structure so for each state, all cell entries are non-negative and all entries sum up to one.
} 


\subsection{Private-values}

In the private value case, for each bidder $i$ the signal $s_{i} \in\{l, h\}$ fully reveals her value. Ambiguity will then play a role in shaping beliefs about the probable bids of the opponent but not about the value of the good. Below we show that the competition effect implies higher bids. The results in this section are closely related to Salo and Weber (1995) and Chen et al (2007) who analyze auctions with ambiguity about the distribution of values in the case of private values. For concreteness, and in order to compare this environment to that of common values, we replicate these results in our setting.

Consider the first-price auction. When $a=1$, this is the standard model and the unique equilibrium has the low type submitting a bid of zero, and the high types mixing between bids in $\left[0, \frac{1}{2}\right]$ according to the distribution $F(b)=\frac{b}{1-b}$. When $a>1$, the low type still bids zero -her value- in equilibrium.

We now consider the high type, when $a>1$. Let $p$ be the belief of the high type of bidder 1 that bidder 2 is a high type. From Table 1 we have that $p=2 \alpha$, and thus given the ePMI constraints for the high type of bidder 1 , given a mixed strategy $F($.$) of the$ other player, the expected utility for some bid $b$ is given by:

$$
\min _{p=2 \alpha, \underline{\alpha}(a) \leq \alpha \leq \bar{\alpha}(a)} \operatorname{Pr}(\text { Bidder } 1 \text { wins })(1-b)=\min _{p=2 \alpha, \underline{\alpha}(a) \leq \alpha \leq \bar{\alpha}(a)}((1-p)+p F(b))(1-b)
$$

From this expression we see that the expected utility depends on beliefs only through the probability of winning. Moreover, it is easy to see that for any $b$, the unique minimizer of utility is the highest $p$ feasible, which is easily derived as $p(a)=2 \bar{\alpha}(a)=1-\frac{1}{2 a}$. Let $\varepsilon(a) \equiv p(a)-\frac{1}{2}$. We can then describe the strategy of the high type in the unique equilibrium as mixing on the interval $\left[0, \frac{1}{2}+\varepsilon(a)\right]$, for $\varepsilon(a)=\frac{1}{2}\left(1-\frac{1}{a}\right)$, according to the distribution $F_{a}(b)=\frac{1}{2 a-1} \frac{b}{1-b}$ which stochastically dominates $F_{a}(b)$ for $a=1$.

Intuitively, ambiguity, together with max-min preferences makes the high bidder believe that the other bidder is more likely a high type and so induces the bidder to bid more aggressively. This is the competition effect; ambiguity over correlation through the prior implies that one can consider different possibilities for the type of the other bidder (and hence her bid). Ambiguity averse bidder will consider the worst case scenario and hence will believe that she faces the toughest possible competition, which will lead her to bid more aggressively, thus increasing the seller's revenue.

In the second-price auction, this effect does not exist as it is still weakly dominant to bid your value. Once the value is known, then the other's bid is not relevant. We then have: ${ }^{11}$

Proposition 1. For private value auctions: (i) In the first-price auction, the equilib-

\footnotetext{
${ }^{11}$ The proofs are in the Appendix.
} 
rium distribution of bids when $a>1$ first order stochastically dominates that of the case in which $a=1$, with the seller's revenues increasing in a. (ii) In the second-price auction, the equilibrium distribution of bids when $a>1$ is the same as that of the case in which $a=1$.

\subsection{Common values}

We now analyse the common values case. In this case an additional effect of ambiguity emerges, as players learn about their value from the equilibrium behaviour of others. Thus, in addition to minimizing their utility by envisaging a low probability of winning, bidders may also choose beliefs that minimize the value of the good conditional on winning. This winner's value effect will sometimes induce an opposite incentive compared with the competition effect; the competition effect can potentially induce bidders to bid more aggressively perceiving a tougher competition; the winner's value effect can induce players to bid less aggressively if they perceive lower valuations upon winning. Bidders can then shed their bids even further than they would absent ambiguity.

Recall that bidders perceive correlation structures as depicted in Table 2, and so ambiguity is over the correlation parameters $\alpha_{0}, \alpha_{1}$. Let us consider the low type of bidder 1. Suppose she uses the equilibrium bid of the low type of bidder 2 , denoted as $b_{a}(l)$, so that $b=b_{a}(l)$. In both the first-price and the second price auction then, her worst-case expected utility is

$$
\min _{\left(\alpha_{0}, \alpha_{1}\right)} \frac{1}{2} \operatorname{Pr}\left(l \mid l,\left(\alpha_{0}, \alpha_{1}\right)\right)\left(E_{\left(\alpha_{0}, \alpha_{1}\right)}(v \mid l, l)-b_{a}(l)\right),
$$

where

$$
\begin{aligned}
\operatorname{Pr}\left(l \mid l,\left(\alpha_{0}, \alpha_{1}\right)\right) & =\alpha_{0}+\alpha_{1} \\
E_{\left(\alpha_{0}, \alpha_{1}\right)}(v \mid l, l) & =\frac{\alpha_{1}}{\alpha_{1}+\alpha_{0}}
\end{aligned}
$$

And so,

$$
\operatorname{Pr}\left(l \mid l,\left(\alpha_{0}, \alpha_{1}\right)\right)\left(E_{\left(\alpha_{0}, \alpha_{1}\right)}(v \mid l, l)-b_{a}(l)\right)=\left(\alpha_{0}+\alpha_{1}\right)\left(\frac{\alpha_{1}}{\alpha_{0}+\alpha_{1}}-b_{a}(l)\right)
$$

Note that the competition effect will imply that to minimize utility one has to minimize the probability of winning, $\alpha_{1}+\alpha_{0}$. On the other hand, the winner's value effect will demand that $\frac{\alpha_{1}}{\alpha_{0}+\alpha_{1}}$ is minimized, which is best achieved when $\alpha_{1}$ is minimized and $\alpha_{0}$ is maximized. When we put the two together, the expression becomes

$$
\alpha_{1}\left(1-b_{a}(l)\right)-\alpha_{0} b_{a}(l)
$$


which implies that the worst case scenario is indeed $\left(\bar{\alpha}_{0}, \underline{\alpha}_{1}\right)$, and so the winner's value effect dominates, as values are chosen to minimize $E_{\left(\alpha_{0}, \alpha_{1}\right)}(v \mid l, l)$. In the first-price action the low type will remain without rent, so that indeed $b=b_{a}(l)=E_{\left(\bar{\alpha}_{0}, \underline{\alpha}_{1}\right)}(v \mid l, l)$. This is also the bid that arises in the second-price auction, where the players use the valuation that is based on both bidders receiving their own signal. We therefore have derived that the bid of the low type is lower than in the case with no ambiguity, in both types of auctions.

What about the high type? The intuition will be clearer in the second-price auction where pure strategies are used (but the results are qualitatively similar as Proposition 2 illustrates). Consider the high type of bidder 1. In the second-price auction, her expected utility when she uses her opponent's high type bid, so that $b=b_{a}(h)>b_{a}(l)$ is

$$
\begin{aligned}
& \min _{\left(\alpha_{0}, \alpha_{1}\right)} \operatorname{Pr}\left(l \mid h,\left(\alpha_{0}, \alpha_{1}\right)\right)\left(E_{\left(\alpha_{0}, \alpha_{1}\right)}(v \mid l, h)-b_{a}(l)\right)+ \\
& (1 / 2) \operatorname{Pr}\left(h \mid h,\left(\alpha_{0}, \alpha_{1}\right)\right)\left(E_{\left(\alpha_{0}, \alpha_{1}\right)}(v \mid h, h)-b_{a}(h)\right),
\end{aligned}
$$

where:

$$
\begin{aligned}
& \operatorname{Pr}\left(l \mid h,\left(\alpha_{0}, \alpha_{1}\right)\right)=1-\alpha_{1}-\alpha_{0}, E_{\left(\alpha_{0}, \alpha_{1}\right)}(v \mid l, h)=\frac{1-q-\alpha_{1}}{1-\alpha_{1}-\alpha_{0}}, \\
& \operatorname{Pr}\left(h \mid h,\left(\alpha_{0}, \alpha_{1}\right)\right)=\alpha_{1}+\alpha_{0}, E_{\left(\alpha_{0}, \alpha_{1}\right)}(v \mid h, h)=\frac{2 q-1+\alpha_{1}}{\alpha_{1}+\alpha_{0}} .
\end{aligned}
$$

Putting these in the expression for the utility, we have:

$$
\min _{\left(\alpha_{0}, \alpha_{1}\right)}\left[\left(\alpha_{1}+\alpha_{0}\right)\left(b_{a}(l)-\frac{1}{2} b_{a}(h)\right)+\frac{1}{2}\left(2 q-1+\alpha_{1}\right)+1-q-\alpha_{1}-b_{a}(l)\right]
$$

The competition and winner's value effects now incorporate the possibility of winning both against the low type and against another high type, as well as the magnitude of the equilibrium bid of the low type. The terms $\frac{1}{2}\left(2 q-1+\alpha_{1}\right)+1-q-\alpha_{1}$ express the expected valuation upon winning. It is easy to see that to minimize utility, one needs to maximize $\alpha_{1}$. The first element, $\left(\alpha_{1}+\alpha_{0}\right)\left(b_{a}(l)-\frac{1}{2} b_{a}(h)\right)$, denotes the expected payment given the two events in which the high type bidder can win. Recall that $b_{a}(l)=E_{\left(\bar{\alpha}_{0}, \underline{\alpha}_{1}\right)}(v \mid l, l)=$ $\frac{\alpha_{1}}{\alpha_{1}+\alpha_{0}}$, which is lower than but related to $\frac{(1-q)^{2}}{(1-q)^{2}+q^{2}}$. Thus, when $q$ is low so that signals are imprecise, $b_{a}(l)$ can be relatively high, implying that to minimize utility one has to minimize $\alpha_{1}$ and $\alpha_{0}$, while when $q$ is high, $b_{a}(l)$ is relatively low, implying that to minimize utility, one has to maximize both. In the case when $q$ is high, both effects are then in tandem implying that the bidder chooses $\left(\bar{\alpha}_{0}, \bar{\alpha}_{1}\right)$. This means that the bid is $b_{a}(h)=E_{\left(\bar{\alpha}_{1}, \bar{\alpha}_{0}\right)}(v \mid h, h)$, which will be lower compared to the case of no ambiguity. Thus the winner's value upon winning against a high type, a more likely event, is indeed lower. In the case of a low $q$, where the competition and winner's value effect may clash, the 
latter dominates implying that the bidder chooses $\left(\underline{\alpha}_{0}, \bar{\alpha}_{1}\right)$. The effect now is more subtle; while this belief minimizes $E_{\left(\alpha_{0}, \alpha_{1}\right)}(v \mid l, h)$, the winner's value when she wins against a low type, it can, for a low $a$, increase $E_{\left(\alpha_{0}, \alpha_{1}\right)}(v \mid h, h)$ beyond the canonical case.

We then have:

Proposition 2. For common value auctions: (i) Second price auctions: In the unique symmetric pure-strategy equilibrium, compared to the canonical model, the low type's bid decreases in a, and the high type's bid decreases in a for either a high enough a or a high enough $q$ and increases in a when both $a$ and $q$ are low. (ii) First-price auctions: For a sufficiently low a, in the unique symmetric equilibrium, compared to the canonical model, the bid of the low type decreases in a, the maximal bid of the high type increases in a, with the average bid of the high type increasing or decreasing depending on $q$.

It is interesting to note that different types use different joint information structures, and thus the model is not equivalent to one in which individuals simply have the wrong belief over correlation. In other words, ambiguity interacts with equilibrium behaviour in a non-trivial manner. This was harder to see in the private values case where the low type always bids her value; however in the common value case, the low type is utilising a different belief to minimise her utility compared with the high type, and moreover, as the result illustrates, the winner's value effect dominates so that the low type (and in most environments also the high type) end up lowering their bid compared with the canonical model.

\subsection{Seller's revenue}

In the case of private values we had seen that the seller's revenue increases with ambiguity over correlation, as bids increase (at least in the first-price auction). The case of common values yields a different result, as can be gleaned from the fact that bids may decrease with $a$. As we show in the Appendix, in the common value case, even when the high type increases her bid, compared to the case of no ambiguity, her expected utility evaluated at the true joint probability distribution will be higher compared to the canonical model. This arises as she increases her bid when $q$ is low, which implies she is more likely to encounter the low type and hence pay the low type's bid which is lower than in the canonical model. Thus, considering the utility of bidders and the seller, we have:

Proposition 3: For common value auctions: (i) Second price auction: The utility of both the high and the low type increases in a and the seller's revenue decreases with a.

(ii) First price auction: The seller's revenue decreases in a. (iii) The seller's revenue is higher in the first-price rather than the second-price auction. 
In the common values case, ambiguity implies that bidders decrease their bids; it therefore allows them to gain a higher utility and implies that the seller's revenue decreases with $a$. As another illustration of this, consider the second-price auction equilibrium in the limit, where all information structures are feasible, and so ambiguity is very large. It is the limit of the unique symmetric pure-strategy equilibria that we characterise in the Appendix, and it is characterised by $b_{a}(l)=0$ and $b_{a}(h)=q \cdot{ }^{12}$ Specifically, the high type believes that signals are fully correlated and so $E_{(q, 1-q)}(v \mid h, h)=E(v \mid h)$. These bids are the lowest among all equilibria and the seller's revenue is therefore substantially lower compared with the canonical model.

This result is different than in the private values case. Intuitively, in the former case ambiguity increased the competitiveness of the bids, and thus these increased. In the common value case, ambiguity in general induced the bidders to minimize their utility by minimising the value they expect to gain when they win, implying lower bids at least on average.

The intuition for the result that the seller's revenue is higher in the first-price auction is as follows. In the second-price auction, an individual's payment depends on the other's bid and as a result, there are more elements in her utility in which her beliefs play a role. For the case of no ambiguity, this implies that she conditions her behaviour on more information, which increases the seller's revenue. ${ }^{13}$ For the case of ambiguity, this implies that individuals have more possibilities to condition on their worst-case beliefs, which decreases the seller's revenue. Intuitively, the competition effect is more pronounced in the first price auction than in the second price auction. This is because in the first price auction the payment is directly related to the bid.

\section{Optimal auctions}

Our analysis here will focus on two results. First, consistent with results in the previous section, the seller's maximum revenue will increase with ambiguity in the private value case but will decrease with ambiguity in the common values case. Second, the type of optimal auction will also change when we switch from private to common values, and specifically, the seller will not necessarily fully insure the bidders.

Indeed the key issue when considering optimal auctions under ambiguity is the level of insurance provided by the seller to the bidders. Under independent private values,

\footnotetext{
${ }^{12}$ This is supported by the low type believing $\alpha_{0}=2 q-1$ and $\alpha_{1}=0$, and the high type believing $\alpha_{0}=q$ and $\alpha_{1}=1-q$.

${ }^{13}$ With private values and ambiguity over the prior, Lo (1998) shows that the first-price auction dominates the second-price auction in some environments.
} 
Bose et al (2006) show that the optimal mechanism fully insures the bidders against ambiguity. In Bose et al (2006) bidders believe that valuations are independently drawn, but face ambiguity regarding the particular distributions of valuations that others have. In contrast, in our model bidders know the marginal distribution of valuations but face ambiguity about the joint distribution of valuations. In Section 4.2 we replicate the full insurance result in our setting. When reporting truthfully, the buyers are fully insured against ambiguity. However, the competition effect makes deviations less attractive, and the seller is able to exploit this by asking the high type to pay more compared to the standard case without ambiguity.

In the common value case, however, the seller does not necessarily provide full insurance. We show that when ambiguity is small or the signals sufficiently precise, the optimal mechanism fully extracts rent (i.e. participation constraints are binding), using side bets with the low type to deter the high type from deviating. These side bets expose the low type to ambiguity, and as a result the seller cannot extract full surplus.

Moreover, the seller will allocate the object to the buyer with the highest signal, which exposes the high type to ambiguity as well. As a result, the seller is then able to insure the high type against the competition effect but not the winner's value effect. Specifically, as we show in Section 4.3, when the two players receive different signals, the seller allocates the object to the high type; the winner's value effect then implies that the high type will undervalue the object, but the seller is able to partially insure the high type by also asking her to pay more when her opponent is low type. Under optimal transfers the high type does not care whether her opponent has received the high or the low signal - that is, the high type is insured against the competition effect. On the other hand when ambiguity is large and the signals imprecise, the seller fully insures the buyers, leaving the high type with positive rents.

\subsection{The seller's problem}

We now formalise the seller's problem. A direct mechanism $(x, t)$ is an allocation rule $x:\{l, h\}^{2} \mapsto[0,1]^{2}$ and a transfer rule $t:\{l, h\}^{2} \mapsto R^{2}$. Let $U_{i}^{\alpha}\left(s^{\prime}, s\right)$ be $i$ 's utility from reporting $s^{\prime}$ when $i$ 's signal is $s$, given that the information structure is $\boldsymbol{\alpha}$. A direct mechanism is maxmin incentive compatible if for all $s \in\{l, h\}$ :

$$
\min _{\boldsymbol{\alpha}} U_{i}^{\alpha}(s, s) \geq \min _{\boldsymbol{\alpha}} U_{i}^{\boldsymbol{\alpha}}\left(s^{\prime}, s\right)
$$

for all $s^{\prime} \in\{l, h\}$. The revelation principle applies in this setting as long as we make the following assumption:

No-hedging: The utility from playing the mixed strategy $\sigma \in \Delta\{l, h\}$ is $\sum_{s^{\prime}} \sigma\left(s^{\prime}\right) \min _{\boldsymbol{\alpha}} U_{i}^{\boldsymbol{\alpha}}\left(s^{\prime}, s\right)$ 
(as opposed to $\min _{\boldsymbol{\alpha}} \sum_{s^{\prime}} \sigma\left(s^{\prime}\right) U_{i}^{\boldsymbol{\alpha}}\left(s^{\prime}, s\right)$ ).

This assumption is standard in the literature on mechanism design with maxmin agents (see for example, Bose et al 2006 or Wolitzky 2016). In what follows, we restrict attention to maxmin incentive compatible direct mechanisms.

\subsection{Private values}

In the private value case, $q_{i}\left(s_{i}=l \mid v_{i}=L\right)=q_{i}\left(s_{i}=h \mid v_{i}=H\right)=1$. For each signal $s \in\{l, h\}$, let $v^{s} \in\{L, H\}$ be the valuation associated with the signal. That is, let $v^{l}=L$ and $v^{h}=H$. Let $p$ denote a buyer's belief that her opponent has received the same signal as her. The ePMI constraints imply, following Table 1 in Section 3 , that $\frac{1}{2 a} \leq p \leq 1-\frac{1}{2 a}$. An optimal mechanism solves the seller's problem:

$$
\max _{x_{i}, t_{i}} \sum_{i=1}^{2} t_{i}(l, l)+t_{i}(l, h)+t_{i}(h, l)+t_{i}(h, h)
$$

subject to:

$$
\begin{gathered}
\min _{p \in\left[\frac{1}{2 a}, 1-\frac{1}{2 a}\right]} p\left[v^{s} x_{i}(s, s)-t_{i}(s, s)\right]+(1-p)\left[v^{s} x_{i}\left(s, s^{\prime}\right)-t_{i}\left(s, s^{\prime}\right)\right] \\
\geq \min _{p \in\left[\frac{1}{2 a}, 1-\frac{1}{2 a}\right]} p\left[v^{s} x_{i}\left(s^{\prime}, s\right)-t_{i}\left(s^{\prime}, s\right)\right]+(1-p)\left[v^{s} x_{i}\left(s^{\prime}, s^{\prime}\right)-t_{i}\left(s^{\prime}, s^{\prime}\right)\right] \\
\min _{p \in\left[\frac{1}{2 a}, 1-\frac{1}{2 a}\right]} p\left[v^{s} x_{i}(s, s)-t_{i}(s, s)\right]+(1-p)\left[v^{s} x_{i}\left(s, s^{\prime}\right)-t_{i}\left(s, s^{\prime}\right)\right] \geq 0
\end{gathered}
$$

for all $i$, and for all $s, s^{\prime} \in\{l, h\}$.

As in Bose et al (2006), when values are private, the seller finds it optimal to fully insure the buyers against ambiguity and is able to extract full surplus when the ambiguity is large:

Proposition 4. When buyers have private values:

(i) Any optimal mechanism is a full insurance mechanism

(ii) The optimal allocation rule depends on $L$ : if $L<\frac{1}{1+a}$, it is not optimal to sell to buyers with the low valuation, but if $L>\frac{1}{1+a}$, the seller always allocates the object.

(iii) The seller's revenue is increasing in ambiguity, and as $a \rightarrow \infty$, converges to full surplus.

In the Appendix, we fully characterise the set of optimal mechanisms under private values. 


\subsection{Common values}

In the common values case, the possible joint information structures are described in Table 2 in Section 3. The seller's problem is:

$$
\max _{x_{i}, t_{i}} \frac{1}{2}\left(q^{2}+(1-q)^{2}\right)\left[\sum_{i=1}^{2} t_{i}(l, l)+t_{i}(h, h)\right]+q(1-q)\left[\sum_{i=1}^{2} t_{i}(l, h)+t_{i}(h, l)\right]
$$

subject to incentive and participation constraints:

$$
\begin{aligned}
& \min _{\alpha_{0}, \alpha_{1}} \alpha_{1} x_{i}(l, l)+\left(1-q-\alpha_{1}\right) x_{i}(l, h)-\left(\alpha_{0}+\alpha_{1}\right) t_{i}(l, l)-\left(1-\alpha_{0}-\alpha_{1}\right) t_{i}(l, h) \\
& \geq \min _{\alpha_{0}, \alpha_{1}} \alpha_{1} x_{i}(h, l)+\left(1-q-\alpha_{1}\right) x_{i}(h, h)-\left(\alpha_{0}+\alpha_{1}\right) t_{i}(h, l)-\left(1-\alpha_{0}-\alpha_{1}\right) t_{i}(h, h) \\
& \min _{\alpha_{0}, \alpha_{1}}\left(1-q-\alpha_{1}\right) x_{i}(h, l)+\left(2 q-1+\alpha_{1}\right) x_{i}(h, h)-\left(1-\alpha_{0}-\alpha_{1}\right) t_{i}(h, l)-\left(\alpha_{0}+\alpha_{1}\right) t_{i}(h, h) \\
& \geq \min _{\alpha_{0}, \alpha_{1}}\left(1-q-\alpha_{1}\right) x_{i}(l, l)+\left(2 q-1+\alpha_{1}\right) x_{i}(l, h)-\left(1-\alpha_{0}-\alpha_{1}\right) t_{i}(l, l)-\left(\alpha_{0}+\alpha_{1}\right) t_{i}(l, h) \\
& \min _{\alpha_{0}, \alpha_{1}} \alpha_{1} x_{i}(l, l)+\left(1-q-\alpha_{1}\right) x_{i}(l, h)-\left(\alpha_{0}+\alpha_{1}\right) t_{i}(l, l)-\left(1-\alpha_{0}-\alpha_{1}\right) t_{i}(l, h) \geq 0 \\
& \min _{\alpha_{0}, \alpha_{1}}\left(1-q-\alpha_{1}\right) x_{i}(h, l)+\left(2 q-1+\alpha_{1}\right) x_{i}(h, h)-\left(1-\alpha_{0}-\alpha_{1}\right) t_{i}(h, l)-\left(\alpha_{0}+\alpha_{1}\right) t_{i}(h, h) \geq 0
\end{aligned}
$$

For a given $a$, the optimal mechanism will depend on two cutoff values of $q$, which we now define. Let $q^{*}(a)$ be the solution to $q^{2}+(1-q)^{2}-\underline{\alpha}_{0}-\underline{\alpha}_{1}=\underline{\alpha}_{0}+\underline{\alpha}_{1}+\bar{\alpha}_{0}+\bar{\alpha}_{1}-1$ that lies between $\frac{1}{2}$ and 1 , and let $q^{* *}(a)$ be the solution to $q^{2}+(1-q)^{2}-\underline{\alpha}_{0}-\underline{\alpha}_{1}=3 q-2+\underline{\alpha}_{1}+\bar{\alpha}_{1}$ that lies between $\frac{1}{2}$ and 1 . We derive the explicit expressions for $q^{*}(a), q^{* *}(a)$ in the Appendix. For $1<a<\infty, q^{*}(a)<q^{* *}(a)$. We then have:

Proposition 5. When buyers have common values:

(i) When $q \leq q^{*}(a)$, an optimal mechanism allocates the good with equal probability for each player disregarding their type, and a transfer of $\frac{1}{2}(1-q)$. The revenue of the seller is $1-q$, both types are fully insured, and the high type earns positive rents.

(ii) When $q \geq q^{* *}(a)$, the optimal mechanism allocates the good to the high type and with equal probability to each player if both are of the same type. Transfers are such that the high type is partially insured, the seller makes side bets with the low type, and the buyers earn no rents.

(iii) When $q^{*}(a)<q<q^{* *}(a)$, the optimal mechanism allocates the good to the high type and with equal probability to each player if both are of the same type. There are no side bets with the low type, both types are partially insured, and the high type earns positive rents. 
(iv) As $a \rightarrow \infty$, both $q^{*}(a)$ and $q^{* *}(a)$ converge to $\frac{1}{2}(3-\sqrt{3})$, and as $a \rightarrow 1$, both $q^{*}(a)$ and $q^{* *}(a)$ converge to $\frac{1}{2}$.

(v) Seller's revenue (weakly, and sometimes strictly) decreases with a.

When $q \leq q^{*}(a)$, an implementation of the optimal mechanism is for the seller to first choose each buyer with equal probability, and then sell to the chosen buyer at price $1-q$. Since the decision to sell is not based on the signal realisation, the good is worth $1-q$ to the low type and $q$ to the high type. Thus, the high type earns positive rents in equilibrium. Note that this mechanism is efficient, and that given the seller's design, ambiguity is not relevant or does not arise in equilibrium.

When $q \geq q^{* *}(a)$, the participation constraint of the high type is binding: it is optimal to fully extract rent. The seller engages in a side bet with the low type to prevent the high type from deviating. Unlike in the classical case, side bets are costly to the seller, so the seller uses the smallest bet that is sufficient to prevent the high type from deviating. To reduce this cost further, the seller allocates the good to the high type when the players receive different signals, which generates endogenous ambiguity over the expected value of the good. The seller is able to partially insure the high type by asking her to pay more when the other player has received a low signal. In this case, the expected payment from the high type is:

$$
T_{i}^{h}=\frac{1}{2}\left(1-(1-q)^{2}\right)-\frac{1}{2}\left(q^{2}-\underline{\alpha}_{0}\right) .
$$

The expected payment from the low type is:

$$
T_{i}^{l}=\frac{\underline{\alpha}_{1}}{2}-\left[q^{2}+(1-q)^{2}-\underline{\alpha}_{0}-\underline{\alpha}_{1}\right] \frac{1-q-\bar{\alpha}_{1}-\underline{\alpha}_{1}}{2\left(\bar{\alpha}_{0}+\bar{\alpha}_{1}+\underline{\alpha}_{0}+\underline{\alpha}_{1}-1\right)} .
$$

The low type chooses $\alpha_{0}$ and $\alpha_{1}$ both to minimise the perceived surplus from winning the object and to maximise the perceived value of the transfers. Note that in the optimal mechanism, the belief of the high type regarding $\alpha_{1}$ is irrelevant. The high type does not care about the type of her opponent: she gets the same utility from any belief about $\alpha_{1}$. In this sense, she is insured against the competition effect. On the other hand she believes that $\alpha_{0}=\underline{\alpha}_{0}$. This gives rise to the winner's value effect: her expectation of the value conditional on winning, $\frac{1-\alpha_{1}}{2-\underline{\alpha}_{0}-\alpha_{1}}$, is lower than true conditional expectation (for any belief $\left.\alpha_{1} \in\left[\underline{\alpha}_{1}, \bar{\alpha}_{1}\right]\right)$. When $a$ converges to 1 , only this case remains.

The intuition why participation constraints must bind when $a$ is small is as follows. If the participation constraint of the high type is slack, the seller can achieve a first order increase in revenue by increasing the payment of the high type. In order to ensure that the incentive constraint is not violated, the seller can increase $t_{i}(l, h)$ and decrease $t_{i}(l, l)$ in such a way that keeps the low type indifferent, but lowers the high type's utility from deviating. Since the low type may have different beliefs to the seller, these changes in 
transfers may decrease the seller's revenue; however, as the ambiguity becomes small, this fall in revenue converges to zero. On the other hand, the increase in revenue from increasing the payment of the high type is fixed.

When $q^{*}(a)<q<q^{* *}(a)$, it is optimal to allocate the good to the high type when the players receive different signals, but it is not optimal to conduct side bets with the low type. Instead, the high type earns positive rents in equilibrium in order to satisfy the incentive constraint. The winner's value effect implies that the buyers underestimate the value of the object conditional on winning. However, the seller partially insures both types, so that the competition effect does not arise. ${ }^{14}$ This interval shrinks as a goes to either 1 or $\infty$.

If buyers are fully insured against ambiguity, then the seller's revenue is constant in ambiguity. However, in the cases where buyers are not fully insured, the seller's revenue is strictly decreasing in ambiguity. The intuition is that as ambiguity increases, the larger winner's value effect forces the seller to decrease transfers. Thus, the seller's revenue is always (weakly) decreasing in ambiguity.

Note that Crémer and McLean (1988) show that some of the conclusions from the analysis of optimal auctions with independent private values are not robust. For example, since surplus extraction is possible when signals are correlated, the optimal mechanism is efficient and leaves no rents to the buyers. Proposition 5 shows that these results continue to hold for $a$ close to $1 .^{15}$ On the other hand, when $a$ is large, it is possible for buyers to earn positive rents in the optimal mechanism. Note that in this environment, it is always possible to fully extract rent (see Renou 2015); however, as we have argued, rent extraction conflicts with full insurance and is not necessarily optimal when ambiguity is large.

\section{Extensions}

We now extend the model of Section 3 in two ways. We first consider the case of many bidders and then consider continuous valuations. In each of these extensions we consider different models of correlation which illustrates the flexibility of the framework. The key results of Section 3 extend to these environments as well: seller's revenue decreases with ambiguity for common value auctions.

\footnotetext{
${ }^{14}$ For the low type, any belief about $\alpha_{0}$ gives the same utility, but she believes that $\alpha_{1}=\underline{\alpha}_{1}$, so that her expected value of the object conditional on winning, $\frac{\underline{\alpha}_{1}}{\alpha_{0}+\underline{\alpha}_{1}}$ is smaller than the true conditional expectation for any belief $\alpha_{0} \in\left[\underline{\alpha}_{0}, \bar{\alpha}_{0}\right]$.

${ }^{15}$ The set of optimal mechanisms when $a=1$ is large. As $a \rightarrow 1$, the optimal mechanism described in Proposition 5, which is the unique symmetric mechanism when $a$ is close to 1 , converges to an optimal mechanism for the case when $a=1$.
} 


\subsection{Many bidders}

Our analysis above focused on the case of two bidders. In this section we consider a model with common values and many bidders. Naturally when extending the model to more than two bidders, many correlation patterns can be considered; we now extend the model in the simplest way that also maintains symmetry and anonymity when a single bidder considers the correlation between herself and all other bidders. Specifically, consider a population of $n$ bidders, who consider distributions of the following form: with probability $\alpha_{0} \in[0, a]$, all bidders receive the same signal in state 0 , and with probability $\alpha_{1} \in[0, a]$, all bidders receive the same signal in state 1 . This signal, $s^{*} \in\{l, h\}$, is drawn from the same marginal distribution as before; that is, $\operatorname{Pr}\left(s^{*}=h \mid v=1\right)=\operatorname{Pr}\left(s^{*}=l \mid v=0\right)=q>\frac{1}{2}$. With the remaining probability, each bidder draws a (conditionally) independent signal,

again, with $\operatorname{Pr}\left(s_{i}=h \mid v=1\right)=\operatorname{Pr}\left(s_{i}=l \mid v=0\right)=q>\frac{1}{2}$ for all $i$. Bidders have ambiguity then over $\alpha_{1}$ and $\alpha_{0}$, as before.

To see that similar considerations are involved, consider a low type. Intuitively, a low type wins only when she faces $n-1$ low types. Minimizing utility implies as before that she will minimize her winning probability in state 1 and maximize it in state 0 . This implies that she sets up the correlation among bidders to be the highest in state 0 , as positive correlation implies the highest probability of having a vector of identical types. Alternatively, she uses the belief that correlation is lowest in state 1; with independent signals the probability of any specific vector of valuations is the lowest. But of course this means that conditioning on a vector of low types implies that state 0 is more likely, inducing a strictly lower bid.

We are then able to show:

Proposition 6: When ambiguity is not too large, small a, there exists a symmetric equilibrium in the first-price auction. Moreover, there exists $\bar{n}$, such that for all $n>\bar{n}$, in this equilibrium the high type conditions her bid on the belief $\left(\alpha_{0}, \alpha_{1}\right)=(0, a)$, and the low type conditions her bid on the belief $\left(\alpha_{0}, \alpha_{1}\right)=(a, 0)$. The (expected) bids of both types are lower than in the independent case, and the seller's revenues decrease with ambiguity.

For the high type (who uses a mixed strategy in equilibrium), the considerations are more complicated as she needs to consider many vectors of types, each affecting both the probability of winning and her valuation. However, for a large $n$, for example in the low state, under independence $\left(\alpha_{0}=0\right)$, the most likely event includes $n(1-q)$ high types and $n q$ low types. Thus perceiving more correlation shifts the weight from this event to the event in which they are all correlated. This increases utility by increasing the probability of paying a lower price in expectations (as one competes with a smaller number of high types). Thus to minimize utility we have to reduce correlation in the low state. Similar 
intuition arises in the high state. This belief implies that the expected bid is lower than in the benchmark independent model, and as a result, seller's revenue is lower as well. While we can verify analytically that the above holds also for small $n$ (e.g., $n=3$ ), an analytical proof for all $n$ can be derived for a large $n$ only.

Note that we focus in this case on a first-price auction as symmetric equilibria in the second-price auction may not exist with discrete types and many bidders. ${ }^{16}$

\section{Continuous signals}

We show that the results of Section 3 are robust to the case of a continuum of signals. In the common-value case, let the state of the world be $v \in\{0,1\}$, with an equal prior. We revert to the case of two bidders, where each now receives a signal $s^{i} \in[0,1]$ about the state of the world. The marginal distributions determining the signals given the state of the world, $g_{v}(s)$ for each player, are known to the players. To simplify, let $g_{0}(s)=2(1-s)$ and $g_{1}(s)=2 s$ (more generally we require in the Appendix that $g_{0}(s)$ is decreasing and $g_{1}(s)$ is increasing, so that that $G_{0}(s)$ is concave and $G_{1}(s)$ is convex). Note that $G_{0}(s)>G_{1}(s)$ for all interior $s$, and hence MLRP is satisfied too.

Individuals have ambiguity over a set of joint distributions in each state $v \in\{0,1\}$. We use a simple set of joint distributions, the F-G-M transformation (copula), which was introduced by Morgenstern in 1956. Specifically, given $g_{v}(s)$, we have:

$$
f_{v}(\mathbf{s})=\left[1+\lambda_{v}\left(2 G_{v}\left(s_{1}\right)-1\right)\left(2 G_{v}\left(s_{2}\right)-1\right)\right] g_{v}\left(s_{1}\right) g_{v}\left(s_{2}\right) .
$$

For this to be a distribution, for any $v$ we need $\left|\lambda_{v}\right| \leq 1 .{ }^{17}$ Note that when $\lambda_{v}>0$ we have positive correlation of signals in state $v$ while when $\lambda_{v}<0$ we have negative correlation. When signals are conditionally independent, we have $\lambda_{v}=0$ for all $v$. Adding ePMI constraints, we then have:

$$
\lambda_{v} \in\left[\frac{1}{a}-1,1-\frac{1}{a}\right] \text { for } v \in\{0,1\} .
$$

We analyze a second-price auction and show very similar results to the one derived in Section 3. Let us first write the utility of a player for each bid $b$. This is

$$
U\left(s^{1}, b\right) \propto \min _{\boldsymbol{\lambda}}\left(\int_{0}^{z}\left(1-b\left(s^{\prime}\right)\right) f_{1}\left(s^{1}, s^{\prime}\right) d s^{\prime}-\int_{0}^{z} b\left(s^{\prime}\right) f_{0}\left(s^{1}, s^{\prime}\right) d s^{\prime}\right)
$$

where $b\left(s^{\prime}\right)$ is the bid used by the other player and $z=b^{-1}(b)$. In the Appendix we show that when the level of ambiguity a is small enough, there exists a symmetric equilibrium

\footnotetext{
${ }^{16}$ This is a general issue that does not relate to our specific model.

${ }^{17}$ This implies that the highest correlation coefficient in this family is $\frac{1}{3}$ in absolute value. See Schucany et al (1978).
} 
in which

$$
b\left(s, \boldsymbol{\lambda}^{*}\right)=E^{\boldsymbol{\lambda}^{*}}(v \mid s, s)=\frac{\left[1+\lambda_{1}^{*}\left(2 G_{1}(s)-1\right)^{2}\right] g_{1}^{2}(s)}{\left[1+\lambda_{1}^{*}\left(2 G_{1}(s)-1\right)^{2}\right] g_{1}^{2}(s)+\left[1+\lambda_{0}^{*}\left(2 G_{0}(s)-1\right)^{2}\right] g_{0}^{2}(s)} .
$$

where $\lambda^{*}(s)$ minimizes the utility for each bid/type. To do so, a player needs to consider how correlation affects both the competition and the winner's value effects, at each state.

As before, low types (specifically, below the median of $\left.G_{0}\right)^{18}$ minimize their utility by postulating the highest possible positive correlation at state 0 and the highest possible negative correlation at state 1 , given the strong winner's value effect. They therefore minimize their valuation upon winning (as they only win when the other player has even lower signals) which implies underbidding.

For high types, positive correlation in state 1 and in state 0 decreases their utility as it implies paying a higher bid (while their probability of winning is relatively high in any case). This implies that they choose the maximum positive correlation in both states. This was also the case in the discrete case and again implies underbidding as conditional on their signal, and as $G_{0}(s)>G_{1}(s)>\frac{1}{2}$ for these types, $f_{0}(s, s)>f_{1}(s, s)$ for any fixed positive $\lambda$. Thus $b\left(s, \boldsymbol{\lambda}^{*}\right)<b(s, 0)$.

We therefore have:

Proposition 7 When the level of ambiguity a is small enough, there exists a symmetric equilibrium in the second-price auction in which which $b\left(s, \lambda^{*}\right)$ is as defined in (1), where $\boldsymbol{\lambda}^{*}(s)$ minimizes the utility of type s in equilibrium, and the seller's revenues decrease with ambiguity.

In the Appendix we generalise for other symmetric $g_{v}(s)$, and characterise a sufficient condition for the marginal distribution function for which revenues decrease more generally. We also consider in the Appendix for compeletness the private value case, where we assume that valuations are drawn from $[0,1]$ according to a uniform distribution, and individuals believe that the joint distribution is $f(\mathbf{v})=1+\lambda\left(2 v_{i}-1\right)\left(2 v_{j}-1\right)$, for $\lambda \in\left[\frac{1}{a}-1,1-\frac{1}{a}\right]$. We characterise a symmetric equilibrium and show that bids in the first-price auction are uniformly higher compared to the case without ambiguity (recall that bids in the second-price auction are not affected by ambiguity).

\section{Conclusion}

We have constructed a framework in which we can analyze ambiguity over correlation in information structures of bidders both in the private and in the common value auctions.

\footnotetext{
${ }^{18}$ The median is important as the F-G-M copula is of the form $2 G-1$.
} 
We have illustrated in this paper that ambiguity over correlation induces different results in the private values versus the common values auctions. Specifically, while seller's revenue increases in the private value auctions, it decreases in the common value auctions. The key insight of the analysis is that in the common value auctions players choose worst-case beliefs that amount to minimising the value of the good conditional on winning. This also leads to the optimal auction providing less than full insurance to bidders.

\section{Appendix}

\subsection{Proofs for Section 3}

\section{Proof of Proposition 1:}

We consider equilibria where the low type bids $L$ and the high type mixes on the support $[L, \bar{b}]$. It can be shown that this equilibrium is unique. ${ }^{19}$ First note that the low type gets zero utility in equilibrium; any bid higher than $L$ yields negative utility, and any bid below $L$ yields 0 utility.

As in the main text, the expected utility from bidding $b$ for the high type is:

$$
\min _{p=2 \alpha, \underline{\alpha}(a) \leq \alpha \leq \bar{\alpha}(a)}((1-p)+p F(b))(1-b),
$$

which is minimised by maximising $\alpha$. As the ePMI constraints are:

$$
\frac{1}{4} a=\underline{\alpha}(a) \leq \alpha \leq \bar{\alpha}(a)=\frac{1}{2}\left(1-\frac{1}{2} a\right),
$$

utility is minimised at $\bar{\alpha}(a)=\frac{1}{2}-\frac{1}{4 a}$ which implies a probability that the opponent has a high value of $1-\frac{1}{2 a}$.

Now we compute the highest bid, $\bar{b}$, in the support of $F(b)$. For the highest bid we have:

\footnotetext{
${ }^{19}$ For uniqueness, first observe that there cannot be pure strategy equilibria, and that in any equilibrium the low type cannot earn positive rents. In any equilibrium, the low type of both players must bid $L$. Suppose that the low type of player 1 bids strictly less than $L$ with positive probability; then the low type of player 2 can get positive rents. But this contradicts the fact that the low type cannot earn positive rents in equilibrium. Thus assume that each low type bids at least $L$ (so the utility of the low type is at most zero). Suppose that at least one low type bids strictly more than $L$ with positive probability, and without loss of generality assume that the low type of player 1 wins the auction with positive probability with a bid strictly higher than $L$ (and receiving strictly negative utility). Then the low type of player 1 has a profitable deviation to bidding $L$ with probability 1 . Thus assume that both low types bid $L$ in equilibrium. The bottom of the support of the mixed strategy for the high type must be $L$, otherwise a high type who is supposed to bid just above the bottom of the support can bid just below the support and reduce expected payment without changing the probability of winning. The indifference condition then uniquely determines the equilibrium.
} 


$$
(1-\bar{b})=\left(\frac{1}{2 a}\right)(1-L) \Leftrightarrow \bar{b}=1-\frac{1}{2 a}(1-L) .
$$

So set $\frac{1}{2}+\varepsilon(a)=1-\frac{1}{2 a}(1-L) \Leftrightarrow \varepsilon(a)=\frac{1}{2}-\frac{1}{2 a}(1-L)>0$. Computing $F^{\frac{1}{2}+\varepsilon(a)}(b)$ we have $F^{\frac{1}{2}+\varepsilon(a)}(b)=\frac{\frac{1}{2}-\varepsilon(a)}{\frac{1}{2}+\varepsilon(a)} \frac{b}{1-b}$ and so $F^{\frac{1}{2}+\varepsilon(a)}(b)$ stochastically dominates $F^{\frac{1}{2}}(b)$ and thus seller's revenues are higher when $a>1$ and increase in $a$.

\section{Proofs of Propositions 2 and $3:^{20}$}

Lemma A. In the common value second-price auction, the unique symmetric purestrategy equilibrium satisfies:

1. The low type bids $b_{a}(l)=E_{\left(\bar{\alpha}_{0}, \underline{\alpha}_{1}\right)}(v \mid l, l)$, a bid that decreases in a;

2. For all $a \leq \frac{q}{1-q}$, there exist cutoffs $\underline{q}, \bar{q}$, with $0.5<\underline{q}<\bar{q}<1$, where:

(a) For $q \in(0.5, \underline{q})$, the high type bids $b_{a}(h)=E_{\left(\underline{\alpha}_{0}, \bar{\alpha}_{1}\right)}(v \mid h, h)$, a bid that increases with $a$.

(b) For $q \in(\bar{q}, 1)$, the high type bids $b_{a}(h)=E_{\left(\bar{\alpha}_{0}, \bar{\alpha}_{1}\right)}(v \mid h, h)$, a bid that decreases with $a$.

(c) For $q \in(\underline{q}, \bar{q})$, the high type bids $b_{a}(h)=E_{\left(\alpha_{0}, \bar{\alpha}_{1}\right)}(v \mid h, h)$ for some $\alpha_{0}$ satisfying $E_{\left(\alpha_{0}, \bar{\alpha}_{1}\right)}(v \mid h, h)=2 E_{\left(\bar{\alpha}_{0}, \underline{\alpha}_{1}\right)}(v \mid l, l)$, a bid that decreases with a.

3. For all $a \geq \bar{a}(q) \geq \frac{q}{1-q}$, the high type bids $b_{a}(h)=E_{\left(\bar{\alpha}_{0}, \bar{\alpha}_{1}\right)}(v \mid h, h) .{ }^{21}$

Proof of Lemma A: We consider monotone equilibria where $b_{a}(l)<b_{a}(h), b_{a}(l)=$ $E_{\left(\alpha_{0}, \alpha_{1}\right)}(v \mid l, l)$ for some $\left(\alpha_{0}, \alpha_{1}\right)$, and $b_{a}(h)=E_{\left(\alpha_{0}^{\prime}, \alpha_{1}^{\prime}\right)}[v \mid h, h]$ for some $\left(\alpha_{0}^{\prime}, \alpha_{1}^{\prime}\right) .{ }^{22}$

Under the "no hedging" condition, deviations to mixed strategies will have lower utility, and thus equilibria are easier to sustain. We use this to characterize equilibria for large values of $a$. Of course all equilibria derived without the "no hedging" condition will remain equilibria under the "no hedging" condition.

\footnotetext{
${ }^{20}$ For simplicity we set $L=0$ but the proof is easily extended to $L>0$.

${ }^{21}$ When $q$ is not too small, $\bar{a}(q)=\frac{q}{1-q}$. When $q$ is sufficiently close to $0.5, \bar{a}(q)>\frac{q}{1-q}$ and symmetric pure-strategy equilibria may not exist in the region $\left[\frac{q}{1-q}, \bar{a}(q)\right]$.

${ }^{22}$ Note that in any pure strategy equilibrium where $b_{a}(l)<b_{a}(h)$, we must have $b_{a}(l)=E_{\left(\alpha_{0}, \alpha_{1}\right)}(v \mid l, l)$ for some $\left(\alpha_{0}, \alpha_{1}\right)$ and $b_{a}(h)=E_{\left(\alpha_{0}^{\prime}, \alpha_{1}^{\prime}\right)}[v \mid h, h]$ for some $\left(\alpha_{0}^{\prime}, \alpha_{1}^{\prime}\right)$. For example, suppose that $b_{a}(l)>$ $E_{\left(\alpha_{0}, \alpha_{1}\right)}(v \mid l, l)$ for all $\left(\alpha_{0}, \alpha_{1}\right)$. Then the low type gets negative utility, which contradicts $b_{a}(l)$ being an equilibrium strategy. Suppose that $b_{a}(l)<E_{\left(\alpha_{0}, \alpha_{1}\right)}(v \mid l, l)$ for all $\left(\alpha_{0}, \alpha_{1}\right)$. Then the equilibrium utility for the low type is $\min _{\left(\alpha_{0}, \alpha_{1}\right)} \frac{1}{2} \operatorname{Pr}\left(l \mid l,\left(\alpha_{0}, \alpha_{1}\right)\right)\left(E_{\left(\alpha_{0}, \alpha_{1}\right)}[v \mid l, l]-b_{a}(l)\right)>0$, but the low type has a profitable deviation to $b_{a}(l)+\varepsilon$, which yields utility $\min _{\left(\alpha_{0}, \alpha_{1}\right)} \operatorname{Pr}\left(l \mid l,\left(\alpha_{0}, \alpha_{1}\right)\right)\left(E_{\left(\alpha_{0}, \alpha_{1}\right)}[v \mid l, l]-b_{a}(l)-\varepsilon\right)$. The argument for the high type is similar.
} 
Consider first the low type. For any bid $b \in\left[b_{a}(l), b_{a}(h)\right)$, we have:

$$
\begin{aligned}
& \min _{\left(\alpha_{0}, \alpha_{1}\right)} \rho \operatorname{Pr}\left(l \mid l,\left(\alpha_{0}, \alpha_{1}\right)\right)\left(E_{\left(\alpha_{0}, \alpha_{1}\right)}[v \mid l, l]-b_{a}(l)\right) \\
& =\min _{\left(\alpha_{0}, \alpha_{1}\right)} \rho \alpha_{1}-\rho\left(\alpha_{1}+\alpha_{0}\right) b_{a}(l)
\end{aligned}
$$

where $\rho=1$ if $b>b_{a}(l)$ and $\frac{1}{2}$ otherwise. This is minimised by $\left(\bar{\alpha}_{0}, \underline{\alpha}_{1}\right)$. Thus the conjectured equilibrium bid is $b_{a}(l)=E_{\left(\bar{\alpha}_{0}, \underline{\alpha}_{1}\right)}(v \mid l, l)$. This will be the case for all equilibria considered.

\section{Equilibrium with over-bidding for the high type:}

Consider now the high type. Consider the case of an equilibrium that satisfies $b_{a}(l)>$ $\frac{1}{2} b_{a}(h)$. Bidding $b=b_{a}(h)$ yields:

$$
\begin{aligned}
& \min _{\left(\alpha_{0}, \alpha_{1}\right)} \operatorname{Pr}\left(l \mid h,\left(\alpha_{0}, \alpha_{1}\right)\right)\left(E_{\left(\alpha_{0}, \alpha_{1}\right)}[v \mid l, h]-b_{a}(l)\right)+ \\
& \frac{1}{2} \operatorname{Pr}\left(h \mid h,\left(\alpha_{0}, \alpha_{1}\right)\right)\left(E_{\left(\alpha_{0}, \alpha_{1}\right)}[v \mid h, h]-b_{a}(h)\right)
\end{aligned}
$$

where the optimal $\left(\alpha_{0}, \alpha_{1}\right)$ is the same as the one that solves

$$
\min _{\left(\alpha_{0}, \alpha_{1}\right)}-\alpha_{0}\left(\frac{b_{a}(h)}{2}-b_{a}(l)\right)-\alpha_{1}\left(\left[1-b_{a}(l)\right]-\frac{\left[1-b_{a}(h)\right]}{2}\right)
$$

Since by assumption $b_{a}(l)>\frac{1}{2} b_{a}(h)$, the payoff is minimised by $\left(\underline{\alpha}_{0}, \bar{\alpha}_{1}\right)$. Thus the conjectured equilibrium bid for the high type is $b_{a}(h)=E_{\left(\underline{\alpha}_{0}, \bar{\alpha}_{1}\right)}[v \mid h, h]$. Note that $E_{\left(\underline{\alpha}_{0}, \bar{\alpha}_{1}\right)}[v \mid h, h]=$ $\frac{2 q-1+a(1-q)^{2}}{a(1-q)^{2}+\frac{1}{a}(1-q)^{2}+2 q-1}$ is increasing in $a$. The equilibrium will hold then only if $E_{\left(\bar{\alpha}_{0}, \underline{\alpha}_{1}\right)}(v \mid l, l)>$ $\frac{1}{2} E_{\left(\underline{\alpha}_{0}, \bar{\alpha}_{1}\right)}[v \mid h, h]$.

Note that the equilibrium payoff will be $\operatorname{Pr}\left(l \mid h,\left(\alpha_{0}, \alpha_{1}\right)\right)\left(E_{\left(\alpha_{0}, \alpha_{1}\right)}[v \mid l, h]-b_{a}(l)\right)$. This has to be non negative and thus under $\left(\underline{\alpha}_{0}, \bar{\alpha}_{1}\right)$, we must have $E_{\left(\underline{\alpha}_{0}, \bar{\alpha}_{1}\right)}[v \mid l, h] \geq b_{a}(l)$.

We now consider deviations.

For the low type, the payoff from any mixed strategy is:

$$
\begin{aligned}
& \min _{\left(\alpha_{0}, \alpha_{1}\right)} \rho_{0} \operatorname{Pr}\left(l \mid l,\left(\alpha_{0}, \alpha_{1}\right)\right)\left(E_{\left(\alpha_{0}, \alpha_{1}\right)}[v \mid l, l]-b_{a}(l)\right)+ \\
& \rho_{1} \operatorname{Pr}\left(h \mid l,\left(\alpha_{0}, \alpha_{1}\right)\right)\left(E_{\left(\alpha_{0}, \alpha_{1}\right)}[v \mid h, l]-b_{a}(h)\right)
\end{aligned}
$$

where $0 \leq \rho_{1} \leq \rho_{0} \leq 1$. Under the information structure $\left(\bar{\alpha}_{0}, \underline{\alpha}_{1}\right)$, the first term is 0 . Note that $E_{\left(\bar{\alpha}_{0}, \underline{\alpha}_{1}\right)}[v \mid h, l]<b_{a}(h)$ is a necessary and sufficient condition for no deviation.

In that case, players bid $b=b_{a}(l)$, use $\left(\bar{\alpha}_{0}, \underline{\alpha}_{1}\right)$ as the information structure, and the equilibrium payoff is 0 . 
Let us now consider the high type. As long as the other player is playing the equilibrium (pure) strategy, the payoff from any mixed strategy is:

$$
\begin{aligned}
& \min _{\left(\alpha_{0}, \alpha_{1}\right)} \rho_{0} \operatorname{Pr}\left(l \mid h,\left(\alpha_{0}, \alpha_{1}\right)\right)\left(E_{\left(\alpha_{0}, \alpha_{1}\right)}[v \mid l, h]-b_{a}(l)\right)+ \\
& \rho_{1} \operatorname{Pr}\left(h \mid h,\left(\alpha_{0}, \alpha_{1}\right)\right)\left(E_{\left(\alpha_{0}, \alpha_{1}\right)}[v \mid h, h]-b_{a}(h)\right),
\end{aligned}
$$

where $0 \leq \rho_{1} \leq \rho_{0} \leq 1$. Under the information structure $\left(\underline{\alpha}_{0}, \bar{\alpha}_{1}\right), E_{\left(\underline{\alpha}_{0}, \bar{\alpha}_{1}\right)}[v \mid h, h]=b_{a}(h)$, which implies that the payoff from the deviation is at most $\operatorname{Pr}\left(l \mid h,\left(\underline{\alpha}_{0}, \bar{\alpha}_{1}\right)\right)\left(E_{\left(\underline{\alpha}_{0}, \bar{\alpha}_{1}\right)}[v \mid l, h]-b_{a}(l)\right)$, which is the equilibrium payoff. Thus, it is not profitable to deviate to any mixed strategy.

Bringing together all the conditions, we now have:

$$
\begin{aligned}
& E_{\left(\underline{\alpha}_{0}, \bar{\alpha}_{1}\right)}[v \mid l, h]>E_{\left(\bar{\alpha}_{0}, \underline{\alpha}_{1}\right)}[v \mid l, l] \\
& E_{\left(\bar{\alpha}_{0}, \underline{\alpha}_{1}\right)}[v \mid h, l]<E_{\left(\underline{\alpha}_{0}, \bar{\alpha}_{1}\right)}[v \mid h, h] \\
& E_{\left(\bar{\alpha}_{0}, \underline{\alpha}_{1}\right)}[v \mid l, l]>\frac{1}{2} E_{\left(\underline{\alpha}_{0}, \bar{\alpha}_{1}\right)}[v \mid h, h] .
\end{aligned}
$$

For $a \leq \frac{q}{1-q}$, these conditions are:

(1) $\frac{1-q-a(1-q)^{2}}{1-\left(\frac{1}{a}(1-q)^{2}+2 q-1\right)-a(1-q)^{2}}-\frac{\frac{1}{a}(1-q)^{2}}{\frac{1}{a}(1-q)^{2}+a(1-q)^{2}+2 q-1}>0$

(2) $\frac{2 q-1+a(1-q)^{2}}{a(1-q)^{2}+\frac{1}{a}(1-q)^{2}+2 q-1}-\frac{1-q-\frac{1}{a}(1-q)^{2}}{1-\left(a(1-q)^{2}+2 q-1\right)-\frac{1}{a}(1-q)^{2}}>0$

(3) $\frac{\frac{1}{a}(1-q)^{2}}{\frac{1}{a}(1-q)^{2}+a(1-q)^{2}+2 q-1}-\frac{1}{2} \frac{2 q-1+a(1-q)^{2}}{a(1-q)^{2}+\frac{1}{a}(1-q)^{2}+2 q-1}>0$

Condition (1) and (2) are satisfied for all $q$, while condition (3) is satisfied for all $q<\underline{q}(a)$.

For $a \geq \frac{q}{1-q}$, condition (3), now $\frac{\frac{1}{a}(1-q)^{2}}{\frac{1}{a}(1-q)^{2}+q-\frac{1}{a} q(1-q)}-\frac{1}{2} \frac{2 q-1+1-q-\frac{1}{a} q(1-q)}{1-q-\frac{1}{a} q(1-q)+\frac{1}{a}(1-q)^{2}+2 q-1}>0$, is not satisfied for $a$ which is above a cutoff $\bar{a}$. Note that allowing for no hedging will not affect the existence of this equilibrium for high $a$.

\section{Equilibria with under-bidding for the high type:}

Next consider the case $b_{a}(l)<\frac{1}{2} b_{a}(h)$. Consider the high type, and assume that the other player is playing the equilibrium strategy $\left(b_{a}(l), b_{a}(h)\right)$.

Bidding $b=b_{a}(h)$ yields:

$$
\begin{aligned}
& \min _{\left(\alpha_{0}, \alpha_{1}\right)} \operatorname{Pr}\left(l \mid h,\left(\alpha_{0}, \alpha_{1}\right)\right)\left(E_{\left(\alpha_{0}, \alpha_{1}\right)}[v \mid l, h]-b_{a}(l)\right)+ \\
& \frac{1}{2} \operatorname{Pr}\left(h \mid h,\left(\alpha_{0}, \alpha_{1}\right)\right)\left(E_{\left(\alpha_{0}, \alpha_{1}\right)}[v \mid h, h]-b_{a}(h)\right)
\end{aligned}
$$

which is like solving

$$
\begin{aligned}
& \min _{\left(\alpha_{0}, \alpha_{1}\right)}-\alpha_{1}+\left(\alpha_{1}+\alpha_{0}\right) b_{a}(l)+\frac{1}{2} \alpha_{1}-\frac{1}{2}\left(\alpha_{1}+\alpha_{0}\right) b_{a}(h) \\
= & \min _{\left(\alpha_{0}, \alpha_{1}\right)}-\alpha_{1}\left(\frac{1}{2}-b_{a}(l)+\frac{1}{2} b_{a}(h)\right)+\alpha_{0}\left(b_{a}(l)-\frac{1}{2} b_{a}(h)\right)
\end{aligned}
$$


For $b_{a}(l)<\frac{1}{2} b_{a}(h)$, this is minimised by $\left(\bar{\alpha}_{0}, \bar{\alpha}_{1}\right)$.

The equilibrium payoff is then $\operatorname{Pr}\left(l \mid h,\left(\bar{\alpha}_{0}, \bar{\alpha}_{1}\right)\right)\left(E_{\left(\bar{\alpha}_{0}, \bar{\alpha}_{1}\right)}[v \mid l, h]-b_{a}(l)\right)$.

We now consider deviations. Let us consider first the high type. The payoff from any mixed strategy is:

$$
\begin{aligned}
& \min _{\left(\alpha_{0}, \alpha_{1}\right)} \rho_{0} \operatorname{Pr}\left(l \mid h,\left(\alpha_{0}, \alpha_{1}\right)\right)\left(E_{\left(\alpha_{0}, \alpha_{1}\right)}[v \mid l, h]-b_{a}(l)\right)+ \\
& \rho_{1} \operatorname{Pr}\left(h \mid h,\left(\alpha_{0}, \alpha_{1}\right)\right)\left(E_{\left(\alpha_{0}, \alpha_{1}\right)}[v \mid h, h]-b_{a}(h)\right),
\end{aligned}
$$

where $0 \leq \rho_{1} \leq \rho_{0} \leq 1$. Under the information structure $\left(\bar{\alpha}_{0}, \bar{\alpha}_{1}\right), E_{\left(\bar{\alpha}_{0}, \bar{\alpha}_{1}\right)}[v \mid h, h]=b_{a}(h)$. Note that this bid decreases with $a$.

Note that in equilibrium we must have $\left(E_{\left(\bar{\alpha}_{0}, \bar{\alpha}_{1}\right)}[v \mid l, h]-E_{\left(\bar{\alpha}_{0}, \underline{\alpha}_{1}\right)}[v \mid l, l]\right) \geq 0$, and that the equilibrium maximises the probability of winning against the low type.

Consider now the low type. Under no "no hedging", we have that the payoff from any mixed strategy is:

$$
\begin{aligned}
& \min _{\left(\alpha_{0}, \alpha_{1}\right)} \rho_{0} \operatorname{Pr}\left(l \mid l,\left(\alpha_{0}, \alpha_{1}\right)\right)\left(E_{\left(\alpha_{0}, \alpha_{1}\right)}[v \mid l, l]-b_{a}(l)\right)+ \\
& \rho_{1} \operatorname{Pr}\left(h \mid l,\left(\alpha_{0}, \alpha_{1}\right)\right)\left(E_{\left(\alpha_{0}, \alpha_{1}\right)}[v \mid h, l]-b_{a}(h)\right)
\end{aligned}
$$

Under the information structure $\left(\bar{\alpha}_{0}, \underline{\alpha}_{1}\right)$, the first term is 0 . Thus a necessary and sufficient condition for the low type not to deviate is $E_{\left(\bar{\alpha}_{0}, \underline{\alpha}_{1}\right)}[v \mid h, l]<E_{\left(\bar{\alpha}_{0}, \bar{\alpha}_{1}\right)}[v \mid h, h]$.

The equilibrium conditions as described above are therefore:

(4) $E_{\left(\bar{\alpha}_{0}, \bar{\alpha}_{1}\right)}[v \mid l, h]>E_{\left(\bar{\alpha}_{0}, \underline{\alpha}_{1}\right)}[v \mid l, l]$

(5) $E_{\left(\bar{\alpha}_{0}, \underline{\alpha}_{1}\right)}[v \mid h, l]<E_{\left(\bar{\alpha}_{0}, \bar{\alpha}_{1}\right)}[v \mid h, h]$

(6) $E_{\left(\bar{\alpha}_{0}, \underline{\alpha}_{1}\right)}[v \mid l, l]<\frac{1}{2} E_{\left(\bar{\alpha}_{0}, \bar{\alpha}_{1}\right)}[v \mid h, h]$.

Conditions (4) and (5) are satisfied for $a \leq \frac{q}{1-q}$, while condition (6) is satisfied for $q>\bar{q}(a)$.

To consider $a>\frac{q}{1-q}$, consider deviations of the low type under the "no hedging" condition. Her utility from a mixed strategy which wins against the low type only with probability $\beta$ and with probability $1-\beta$ wins against the low type with probability 1 as well as against the high type with probability $\frac{1}{2}$ is:

$$
\begin{aligned}
& \beta \min _{\left(\alpha_{0}, \alpha_{1}\right)} \operatorname{Pr}\left(l \mid l,\left(\alpha_{0}, \alpha_{1}\right)\right)\left(E_{\left(\alpha_{0}, \alpha_{1}\right)}[v \mid l, l]-b_{a}(l)\right) \\
& +(1-\beta) \min _{\left(\alpha_{0}, \alpha_{1}\right)}\left(\operatorname{Pr}\left(l \mid l,\left(\alpha_{0}, \alpha_{1}\right)\right)\left(E_{\left(\alpha_{0}, \alpha_{1}\right)}[v \mid l, l]-b_{a}(l)\right)+\right. \\
& \left.\frac{1}{2} \operatorname{Pr}\left(h \mid l,\left(\alpha_{0}, \alpha_{1}\right)\right)\left(E_{\left(\alpha_{0}, \alpha_{1}\right)}[v \mid h, l]-b_{a}(h)\right)\right)
\end{aligned}
$$

Note that $\arg \min _{\left(\alpha_{0}, \alpha_{1}\right)} \operatorname{Pr}\left(l \mid l,\left(\alpha_{0}, \alpha_{1}\right)\right)\left(E_{\left(\alpha_{0}, \alpha_{1}\right)}[v \mid l, l]-b_{a}(l)\right)$ is $\left(\bar{\alpha}_{0}, \underline{\alpha}_{1}\right)$, and thus this 
part of the utility is 0 , and that

$$
\begin{aligned}
& \arg \min _{\left(\alpha_{0}, \alpha_{1}\right)} \operatorname{Pr}\left(l \mid l,\left(\alpha_{0}, \alpha_{1}\right)\right)\left(E_{\left(\alpha_{0}, \alpha_{1}\right)}[v \mid l, l]-b_{a}(l)\right)+\frac{1}{2} \operatorname{Pr}\left(h \mid l,\left(\alpha_{0}, \alpha_{1}\right)\right)\left(E_{\left(\alpha_{0}, \alpha_{1}\right)}[v \mid h, l]-b_{a}(h)\right) \\
= & \left(\underline{\alpha}_{0}, \underline{\alpha}_{1}\right)
\end{aligned}
$$

A necessary and sufficient condition under the no hedging condition is for the above utility to be lower than 0 , the equilibrium utility.

Thus, for $a>\frac{q}{1-q}$, the equilibrium conditions are:

(4) $E_{\left(\bar{\alpha}_{0}, \bar{\alpha}_{1}\right)}[v \mid l, h]>E_{\left(\bar{\alpha}_{0}, \underline{\alpha}_{1}\right)}[v \mid l, l]$

$\left(5^{*}\right) \operatorname{Pr}\left(l \mid l,\left(\underline{\alpha}_{0}, \underline{\alpha}_{1}\right)\right)\left(E_{\underline{\alpha}_{0}, \underline{\alpha}_{1}}[v \mid l, l]-b_{a}(l)\right)+\frac{1}{2} \operatorname{Pr}\left(h \mid l,\left(\underline{\alpha}_{0}, \underline{\alpha}_{1}\right)\right)\left(E_{\left(\underline{\alpha}_{0}, \underline{\alpha}_{1}\right)}[v \mid h, l]-b_{a}(h)\right)<$

(6) $E_{\left(\bar{\alpha}_{0}, \underline{\alpha}_{1}\right)}[v \mid l, l]<\frac{1}{2} E_{\left(\bar{\alpha}_{0}, \bar{\alpha}_{1}\right)}[v \mid h, h]$.

This equilibrium exists when $a>\bar{a}(q) \geq \frac{q}{1-q}$, where $\bar{a}(q)>\frac{q}{1-q}$ for a low enough $q$ but $\bar{a}(q)=\frac{q}{1-q}$ otherwise.

Note also that the equilibrium converges to the equilibrium in the limit where all information structures are allowed. To see the limit equilibrium, suppose that $b_{a}(l)=0$. For the low type we minimize $\alpha_{1}$ at 0 and set $\alpha_{0}=2 q-1$ (which she is indifferent to) and hence $E(v \mid l, l)=0$. We are therefore in the case in which $b_{a}(l)<\frac{1}{2} b_{a}(h)$ and hence the high type uses $\alpha_{0}=q$ and $\alpha_{1}=1-q$. As a result, $b_{a}(h)=q=E(v \mid h)<E(v \mid h, h)$. This yields to the seller the lowest revenue.

Finally, consider the case $b_{a}(l)=\frac{1}{2} b_{a}(h)$. We will show that this equilibrium holds for $a<\frac{q}{1-q}$, for values $\underline{q}(a)<q<\bar{q}(a)$.

Let $a$ and $q$ satisfy: $\frac{1}{2} E_{\left(\bar{\alpha}_{0}, \bar{\alpha}_{1}\right)}[v \mid h, h]<E_{\left(\bar{\alpha}_{0}, \underline{\alpha}_{1}\right)}[v \mid l, l]<\frac{1}{2} E_{\left(\underline{\alpha}_{0}, \bar{\alpha}_{1}\right)}[v \mid h, h]$

Consider the high type, and assume that the other player is playing the equilibrium strategy $\left(b_{a}(l), b_{a}(h)\right)$.

Bidding $b=b_{a}(h)$ yields:

$\min _{\left(\alpha_{0}, \alpha_{1}\right)} \operatorname{Pr}\left(l \mid h,\left(\alpha_{0}, \alpha_{1}\right)\right)\left(E_{\left(\alpha_{0}, \alpha_{1}\right)}[v \mid l, h]-b_{a}(l)\right)+\frac{1}{2} \operatorname{Pr}\left(h \mid h,\left(\alpha_{0}, \alpha_{1}\right)\right)\left(E_{\left(\alpha_{0}, \alpha_{1}\right)}[v \mid h, h]-b_{a}(h)\right)$

Since $b_{a}(l)=\frac{1}{2} b_{a}(h)$, both $\left(\underline{\alpha}_{0}, \bar{\alpha}_{1}\right)$ and $\left(\bar{\alpha}_{0}, \bar{\alpha}_{1}\right)$ achieve the minimum payoff.

The payoff from any mixed strategy is:

$$
\begin{aligned}
& \min _{\left(\alpha_{0}, \alpha_{1}\right)} \rho_{0} \operatorname{Pr}\left(l \mid h,\left(\alpha_{0}, \alpha_{1}\right)\right)\left(E_{\left(\alpha_{0}, \alpha_{1}\right)}[v \mid l, h]-b_{a}(l)\right)+ \\
& \rho_{1} \operatorname{Pr}\left(h \mid h,\left(\alpha_{0}, \alpha_{1}\right)\right)\left(E_{\left(\alpha_{0}, \alpha_{1}\right)}[v \mid h, h]-b_{a}(h)\right)
\end{aligned}
$$

Since $\operatorname{Pr}_{\left(\alpha_{0}, \alpha_{1}\right)}(l \mid h)\left(E_{\left(\alpha_{0}, \alpha_{1}\right)}[v \mid l, h]-b_{a}(l)\right)>0$ for any $\left(\alpha_{0}, \alpha_{1}\right)$ and increasing $\rho_{0}$ relaxes the constraint on $\rho_{1}$, it is without loss to set $\rho_{0}=1$. Using the fact that $b_{a}(l)=\frac{1}{2} b_{a}(h)$, 
the payoff becomes:

$$
\begin{aligned}
& \min _{\left(\alpha_{0}, \alpha_{1}\right)} \operatorname{Pr}\left(l \mid h,\left(\alpha_{0}, \alpha_{1}\right)\right)\left(E_{\left(\alpha_{0}, \alpha_{1}\right)}[v \mid l, h]-b_{a}(l)\right)+ \\
& \rho_{1} \operatorname{Pr}\left(h \mid h,\left(\alpha_{0}, \alpha_{1}\right)\right)\left(E_{\left(\alpha_{0}, \alpha_{1}\right)}[v \mid h, h]-2 b_{a}(l)\right) \\
& =\min _{\left(\alpha_{0}, \alpha_{1}\right)} 1-b_{a}(l)-q+\rho_{1}(2 q-1)+\alpha_{0} b_{a}(l)\left(1-2 \rho_{1}\right)+\alpha_{1}\left(\rho_{1}\left[1-2 b_{a}(l)\right]-\left[1-b_{a}(l)\right]\right)
\end{aligned}
$$

The payoff is minimised by $\left(\underline{\alpha}_{0}, \bar{\alpha}_{1}\right)$ when $\rho_{1} \leq \frac{1}{2}$ and $\left(\bar{\alpha}_{0}, \bar{\alpha}_{1}\right)$ when $\rho_{1} \geq \frac{1}{2}$.

Suppose that $\rho_{1}>\frac{1}{2}$. Then under $\left(\bar{\alpha}_{0}, \bar{\alpha}_{1}\right)$, the payoff is lower than when $\rho_{1}=\frac{1}{2}$, since $E_{\left(\bar{\alpha}_{0}, \bar{\alpha}_{1}\right)}[v \mid h, h]<b_{a}(h)$. If $\rho_{1}<\frac{1}{2}$, then under $\left(\underline{\alpha}_{0}, \bar{\alpha}_{1}\right)$, the payoff is lower than when $\rho_{1}=\frac{1}{2}$, since $E_{\left(\underline{\alpha}_{0}, \bar{\alpha}_{1}\right)}[v \mid h, h]>b_{a}(h)$. Thus, for $\rho_{1} \neq \frac{1}{2}$, the payoff must be lower than when $\rho_{1}=\frac{1}{2}$, which is the equilibrium payoff.

Now consider the low type. As before, the equilibrium payoff is 0 . The payoff from any mixed strategy is:

$$
\begin{aligned}
& \min _{\left(\alpha_{0}, \alpha_{1}\right)} \rho_{0} \operatorname{Pr}\left(l \mid l,\left(\alpha_{0}, \alpha_{1}\right)\right)\left(E_{\left(\alpha_{0}, \alpha_{1}\right)}[v \mid l, l]-b_{a}(l)\right)+ \\
& \rho_{1} \operatorname{Pr}\left(h \mid l,\left(\alpha_{0}, \alpha_{1}\right)\right)\left(E_{\left(\alpha_{0}, \alpha_{1}\right)}[v \mid h, l]-b_{a}(h)\right)
\end{aligned}
$$

Under the information structure $\left(\bar{\alpha}_{0}, \underline{\alpha}_{1}\right)$, the first term is 0 and the second term is negative if $E_{\left(\bar{\alpha}_{0}, \underline{\alpha}_{1}\right)}[v \mid h, l]<b_{a}(h)$.

So for this to hold we need $\frac{1}{2} E_{\left(\bar{\alpha}_{0}, \bar{\alpha}_{1}\right)}[v \mid h, h]<E_{\left(\bar{\alpha}_{0}, \underline{\alpha}_{1}\right)}[v \mid l, l]<\frac{1}{2} E_{\left(\underline{\alpha}_{0}, \bar{\alpha}_{1}\right)}[v \mid h, h]$ and $\frac{1}{2} E_{\left(\bar{\alpha}_{0}, \underline{\alpha}_{1}\right)}[v \mid h, l]<E_{\left(\bar{\alpha}_{0}, \underline{\alpha}_{1}\right)}[v \mid l, l]$, which is satisfied for the range of $q$ s considered.

Equilibria in the first price auction: We will now show that the maximum bid increases in $a$, and that the minimum bid decreases in $a$, for $a$ close to 1 .

Consider a low type. For any $b_{a}(l)$, this type's expected utility is perceived as

$$
\begin{aligned}
& \min _{\left(\alpha_{0}, \alpha_{1}\right)}\left(\alpha_{0}+\alpha_{1}\right)\left(\frac{\alpha_{1}}{\alpha_{0}+\alpha_{1}}-b_{a}(l)\right) \\
= & \min _{\left(\alpha_{0}, \alpha_{1}\right)} \alpha_{0}\left(-b_{a}(l)\right)+\alpha_{1}\left(1-b_{a}(l)\right)
\end{aligned}
$$

which is resolved by setting $\alpha_{0}$ to be the highest possible value and $\alpha_{1}$ to be the lowest possible value, given the ePMI constraints. Therefore for $a$ sufficiently close to 1 , the solution is $\left(\bar{\alpha}_{0}, \underline{\alpha}_{1}\right)$.

Note that the low type cannot earn positive rents in equilibrium, and thus we set:

$$
b_{a}(l)=E_{\left(\bar{\alpha}_{0}, \underline{\alpha}_{1}\right)}(v \mid l, l)=\frac{\frac{1}{a}(1-q)^{2}}{\frac{1}{a}(1-q)^{2}+a(1-q)^{2}+2 q-1}<\frac{(1-q)^{2}}{(1-q)^{2}+q^{2}}
$$

Taking a derivative of $E_{\left(\bar{\alpha}_{0}, \underline{\alpha}_{1}\right)}(v \mid l, l)$ with respect to $a$, it is straightforward to see that it is negative. Thus the bid of the low type decreases with $a$. We will establish later that this type will not want to use any other bid given the behaviour of the high type. 
Now let us consider the high type. Wlog we can consider a mixed strategy with support on $\left[b_{a}(l), \bar{b}_{a}(h)\right]$, as bidding less than $b_{a}(l)$ will provide a zero utility.

First let us consider a bid just above $b_{a}(l)$ which allows the individual to win against the low type only. We then need to solve the following,

$$
\begin{aligned}
& \min _{\left(\alpha_{0}, \alpha_{1}\right)} \operatorname{Pr}\left(l \mid h,\left(\alpha_{0}, \alpha_{1}\right)\right)\left(E_{\left(\alpha_{0}, \alpha_{1}\right)}(v \mid h, l)-b_{a}(l)\right) \\
= & \min _{\left(\alpha_{0}, \alpha_{1}\right)}\left(q-\alpha_{0}\right)\left(-b_{a}(l)\right)+\left(1-q-\alpha_{1}\right)\left(1-b_{a}(l)\right),
\end{aligned}
$$

which yields the need to maximize $\alpha_{1}$ and to minimize $\alpha_{0}$. The solution is $\left(\underline{\alpha}_{0}, \bar{\alpha}_{1}\right)$. Note that this bid provides a utility of $\operatorname{Pr}\left(l \mid h,\left(\underline{\alpha}_{0}, \bar{\alpha}_{1}\right)\right)\left(E_{\left(\underline{\alpha}_{0}, \bar{\alpha}_{1}\right)}(v \mid h, l)-E_{\left(\bar{\alpha}_{0}, \underline{\alpha}_{1}\right)}(v \mid l, l)\right)$, and that $\bar{\alpha}_{0}+\underline{\alpha}_{1}=\underline{\alpha}_{0}-\bar{\alpha}_{1}$.

We now consider the highest bid in the support, $\bar{b}_{a}(h)$. Such bid implies winning for sure and thus unambiguous gain of $E(v \mid h)$. To be indifferent, this bid has to satisfy

$$
\begin{aligned}
& E(v \mid h)-\bar{b}_{a}(h) \\
= & \operatorname{Pr}\left(l \mid h,\left(\underline{\alpha}_{0}, \bar{\alpha}_{1}\right)\right)\left(E_{\left(\underline{\alpha}_{0}, \bar{\alpha}_{1}\right)}(v \mid h, l)-E_{\left(\bar{\alpha}_{0}, \underline{\alpha}_{1}\right)}(v \mid l, l)\right)
\end{aligned}
$$

Thus:

$$
\begin{aligned}
\bar{b}_{a}(h) & =\operatorname{Pr}_{\left(\underline{\alpha}_{0}, \bar{\alpha}_{1}\right)}(h \mid h) E_{\left(\underline{\alpha}_{0}, \bar{\alpha}_{1}\right)}(v \mid h, h)+\operatorname{Pr}\left(l \mid h,\left(\underline{\alpha}_{0}, \bar{\alpha}_{1}\right)\right) E_{\left(\bar{\alpha}_{0}, \underline{\alpha}_{1}\right)}(v \mid l, l) \\
& =2 q-1+\bar{\alpha}_{1}+\left(1-\underline{\alpha}_{0}-\bar{\alpha}_{1}\right) \frac{\underline{\alpha}_{1}}{\bar{\alpha}_{0}+\underline{\alpha}_{1}} \\
& =2 q-1+\bar{\alpha}_{1}+\frac{\underline{\alpha}_{1}}{\bar{\alpha}_{0}+\underline{\alpha}_{1}}-\underline{\alpha}_{1} \\
& =2 q-1+a(1-q)^{2}+\frac{\frac{1}{a}(1-q)^{2}}{a(1-q)^{2}+2 q-1+\frac{1}{a}(1-q)^{2}}-\frac{1}{a}(1-q)^{2} \\
& =2 q-1+(1-q)^{2}\left(a-\frac{1}{a}+\frac{\frac{1}{a}}{\left(a+\frac{1}{a}-1\right)(1-q)^{2}+q^{2}}\right)
\end{aligned}
$$

Note that the derivative of $a-\frac{1}{a}+\frac{\frac{1}{a}}{\left(\frac{1}{a}+a-1\right)(1-q)^{2}+q^{2}}$, evaluated at $a=1$, is $\frac{(2 q-1)^{2}}{2 q^{2}-2 q+1}>0$. Thus the maximum bid increases in $a$.

We now continue to characterize the equilibrium distribution. Let us consider the worst case scenario in terms of utility for some distribution $F(b)$ with density $f(b)$. The expected utility is

$$
\begin{aligned}
& \int_{b} f(b)\left[\operatorname{Pr}\left(l \mid h,\left(\alpha_{0}, \alpha_{1}\right)\right)\left(E_{\left(\alpha_{0}, \alpha_{1}\right)}(v \mid h, l)-b\right)+\right. \\
& \left.\operatorname{Pr}\left(h \mid h,\left(\alpha_{0}, \alpha_{1}\right)\right) F(b)\left(E_{\left(\alpha_{0}, \alpha_{1}\right)}(v \mid h, h)-b\right)\right] d b \\
= & \int_{b} f(b)\left[E_{\left(\alpha_{0}, \alpha_{1}\right)}(v \mid h)-b-\right. \\
& \left.(1-F(b)) \operatorname{Pr}\left(h \mid h,\left(\alpha_{0}, \alpha_{1}\right)\right)\left(E_{\left(\alpha_{0}, \alpha_{1}\right)}(v \mid h, h)-b\right)\right] d b
\end{aligned}
$$


To choose the information structure to minimize utility, we maximise

$$
\begin{aligned}
& \operatorname{Pr}\left(h \mid h,\left(\alpha_{0}, \alpha_{1}\right)\right)\left(E_{\left(\alpha_{0}, \alpha_{1}\right)}(v \mid h, h)-b\right) \\
= & (2 q-1)+\left(\alpha_{0}(-b)+\alpha_{1}(1-b)\right)
\end{aligned}
$$

and the solution is therefore, for all $b$, to maximize $\alpha_{1}$ and to minimize $\alpha_{0}$. $F(b)$ is characterized by using the indifference condition under the belief $\left(\underline{\alpha}_{0}, \bar{\alpha}_{1}\right)$ :

$$
\begin{aligned}
& \operatorname{Pr}\left(l \mid h,\left(\underline{\alpha}_{0}, \bar{\alpha}_{1}\right)\right)\left(E_{\left(\underline{\alpha}_{0}, \bar{\alpha}_{1}\right)}(v \mid h, l)-b\right)+ \\
& \operatorname{Pr}\left(h \mid h,\left(\underline{\alpha}_{0}, \bar{\alpha}_{1}\right)\right) F(b)\left(E_{\left(\underline{\alpha}_{0}, \bar{\alpha}_{1}\right)}(v \mid h, h)-b\right) \\
= & \operatorname{Pr}\left(l \mid h,\left(\underline{\alpha}_{0}, \bar{\alpha}_{1}\right)\right)\left(E_{\left(\underline{\alpha}_{0}, \bar{\alpha}_{1}\right)}(v \mid h, l)-b_{a}(l)\right)
\end{aligned}
$$

implying that

$$
F_{a}(b)=\frac{\operatorname{Pr}\left(l \mid h,\left(\underline{\alpha}_{0}, \bar{\alpha}_{1}\right)\right)\left(b-b_{a}(l)\right)}{\operatorname{Pr}\left(h \mid h,\left(\underline{\alpha}_{0}, \bar{\alpha}_{1}\right)\right)\left(E_{\left(\underline{\alpha}_{0}, \bar{\alpha}_{1}\right)}(v \mid h, h)-b\right)} .
$$

We complete the equilibrium characterization by showing that given the strategy of the high type, the low type will not deviate.

For the low type, bidding any $b$ above $b_{a}(l)$, we choose the belief to minimize expected utility:

$$
\begin{aligned}
& \min _{\left(\alpha_{1}, \alpha_{0}\right)} \operatorname{Pr}\left(l \mid l,\left(\alpha_{0}, \alpha_{1}\right)\right)\left(E_{\left(\alpha_{0}, \alpha_{1}\right)}(v \mid l, l)-b\right)+ \\
& \operatorname{Pr}\left(h \mid l,\left(\alpha_{0}, \alpha_{1}\right)\right) F_{a}(b)\left(E_{\left(\alpha_{0}, \alpha_{1}\right)}(v \mid l, h)-b\right) \\
= & \min _{\left(\alpha_{0}, \alpha_{1}\right)}(\underline{v}-b)\left(\alpha_{0}\left(1-F_{a}(b)\right)+F_{a}(b) q\right)+(\bar{v}-b)\left(\alpha_{1}\left(1-F_{a}(b)\right)+F_{a}(b)(1-q)\right)
\end{aligned}
$$

As $F_{a}(b) \leq 1$, the solution is $\left(\underline{\alpha}_{1}, \bar{\alpha}_{0}\right)$.

This gives us a utility of $\operatorname{Pr}\left(l \mid l,\left(\bar{\alpha}_{0}, \underline{\alpha}_{1}\right)\right)\left(E_{\left(\bar{\alpha}_{0}, \underline{\alpha}_{1}\right)}(v \mid l, l)-b\right)+$

$\operatorname{Pr}\left(h \mid l,\left(\bar{\alpha}_{0}, \underline{\alpha}_{1}\right)\right) \frac{\operatorname{Pr}\left(l \mid h,\left(\underline{\alpha}_{0}, \bar{\alpha}_{1}\right)\right)\left(b-E_{\left(\bar{\alpha}_{0}, \underline{\alpha}_{1}\right)}(v \mid l, l)\right)}{\operatorname{Pr}\left(h \mid h,\left(\underline{\alpha}_{0}, \bar{\alpha}_{1}\right)\right)\left(E_{\left(\underline{\alpha}_{0}, \bar{\alpha}_{1}\right)}(v \mid h, h)-b\right)}\left(E_{\left(\bar{\alpha}_{0}, \underline{\alpha}_{1}\right)}(v \mid l, h)-b\right)=$

$\operatorname{Pr}\left(h \mid l,\left(\bar{\alpha}_{0}, \underline{\alpha}_{1}\right)\right)\left(b-E_{\left(\bar{\alpha}_{0}, \underline{\alpha}_{1}\right)}(v \mid l, l)\right)\left(\frac{\operatorname{Pr}\left(l \mid h,\left(\underline{\alpha}_{0}, \bar{\alpha}_{1}\right)\right)}{\operatorname{Pr}\left(h \mid h,\left(\underline{\alpha}_{0}, \bar{\alpha}_{1}\right)\right)} \frac{\left(E_{\left(\bar{\alpha}_{0}, \underline{\alpha}_{1}\right)}(v \mid l, h)-b\right)}{\left(E_{\left(\underline{\alpha}_{0}, \bar{\alpha}_{1}\right)}(v \mid h, h)-b\right)}-\frac{\operatorname{Pr}\left(l \mid l,\left(\bar{\alpha}_{0}, \underline{\alpha}_{1}\right)\right)}{\operatorname{Pr}\left(h \mid l,\left(\bar{\alpha}_{0}, \underline{\alpha}_{1}\right)\right)}\right)$. Note that $E_{\left(\bar{\alpha}_{0}, \underline{\alpha}_{1}\right)}(v \mid l, h)=\frac{1-q-\frac{1}{a}(1-q)^{2}}{1-\bar{\alpha}_{0}-\underline{\alpha}_{1}}<\frac{2 q-1+a(1-q)^{2}}{\underline{\alpha}_{0}+\bar{\alpha}_{1}}=E_{\left(\underline{\alpha}_{0}, \bar{\alpha}_{1}\right)}(v \mid h, h)$, for $a$ sufficiently close to 1 , and that $\frac{\operatorname{Pr}_{\left(\underline{\alpha}_{0}, \bar{\alpha}_{1}\right)}(l \mid h)}{\operatorname{Pr}\left(\underline{\alpha}_{0}, \bar{\alpha}_{1}\right)}(h \mid h)=\frac{1-\underline{\alpha}_{0}-\bar{\alpha}_{1}}{\underline{\alpha}_{0}, \bar{\alpha}_{1}}<\frac{\left(\bar{\alpha}_{0}+\underline{\alpha}_{1}\right)}{1-\left(\bar{\alpha}_{0}+\underline{\alpha}_{1}\right)}=\frac{\operatorname{Pr}\left(l \mid l,\left(\bar{\alpha}_{0}, \underline{\alpha}_{1}\right)\right)}{\operatorname{Pr}\left(h \mid l,\left(\bar{\alpha}_{0}, \underline{\alpha}_{1}\right)\right)}$, as $\bar{\alpha}_{0}+\underline{\alpha}_{1}=\bar{\alpha}_{1}+\underline{\alpha}_{0}>\frac{1}{2}$ for all $a$. Thus the utility is negative and the low type does not deviate.

Seller's revenue in the first price auction. Expected payment to seller, $\Pi$, is given by the linear combination of receiving the bid of the low type (when both are $l$ ), the expected bid of the high type (when only one is $h$ ), and the maximum bid of the two $h$ types:

$$
\Pi=\operatorname{Pr}(l, l) E_{\left(\bar{\alpha}_{0}, \underline{\alpha}_{1}\right)}[v \mid l, l]+2 \operatorname{Pr}(l, h) E\left[b_{a}(h)\right]+\operatorname{Pr}(h, h) E\left[\max _{i=1,2} b_{a}^{i}(h)\right]
$$


For expositional purposes we write this as

$$
\Pi=\frac{1}{2} \gamma y+(1-\gamma) \int_{y}^{\alpha x+(1-\alpha) y} b f_{a}(b) d b+\frac{\gamma}{2} \int_{y}^{\alpha x+(1-\alpha) y} b 2 f(b) F_{a}(b) d b
$$

where:

$\gamma=\operatorname{Pr}(l \mid l)$, according to the true (independent) information structure, $\alpha=\operatorname{Pr}\left(l \mid l,\left(\underline{\alpha}_{0}, \bar{\alpha}_{1}\right)\right)$ according to the belief of the high bidder, $\left(\underline{\alpha}_{0}, \bar{\alpha}_{1}\right), x=E_{\left(\underline{\alpha}_{0}, \bar{\alpha}_{1}\right)}[v \mid h, h], y=E_{\left(\bar{\alpha}_{0}, \underline{\alpha}_{1}\right)}[v \mid l, l]=$ $b_{a}(l)$. We therefore also have $\bar{b}_{a}(h)=\alpha x+(1-\alpha) y, F_{a}(b)=\frac{1-\alpha}{\alpha} \frac{b-y}{x-b}$ and $f_{a}(b)=$ $\frac{1}{\alpha} \frac{1-\alpha}{(x-b)^{2}}(x-y)$.

We start by some preliminary results:

\section{Fact 1}

$$
\frac{\partial x}{\partial a}=-\frac{\partial y}{\partial a}>0
$$

\section{Proof of Fact 1:}

Note that $x=E_{\left(\underline{\alpha}_{0}, \bar{\alpha}_{1}\right)}(v \mid h, h)=\frac{2 q-1+a(1-q)^{2}}{q^{2}-\left(1-\frac{1}{a}\right)(1-q)^{2}+a(1-q)^{2}}$

$$
\begin{aligned}
& \frac{\partial x}{\partial a}=\frac{\partial}{\partial a}\left(\frac{2 q-1+a(1-q)^{2}}{q^{2}-\left(1-\frac{1}{a}\right)(1-q)^{2}+a(1-q)^{2}}\right)=(q-1)^{2} \frac{2 a+2 q+2 a q^{2}-4 a q-1}{\left(a^{2} q^{2}-2 a^{2} q+a^{2}+2 a q-a+q^{2}-2 q+1\right)^{2}}>0 \\
& \frac{\partial y}{\partial a}=\frac{\partial}{\partial a} \frac{\frac{1}{a}(1-q)^{2}}{2 q-1+a(1-q)^{2}+\frac{1}{a}(1-q)^{2}}=-(q-1)^{2} \frac{2 a+2 q+2 a q^{2}-4 a q-1}{\left(a^{2} q^{2}-2 a^{2} q+a^{2}+2 a q-a+q^{2}-2 q+1\right)^{2}} .
\end{aligned}
$$

For low $q$ and low $a, \operatorname{Pr}(l \mid h)>\frac{1}{2} \operatorname{Pr}(h \mid h), \frac{\partial x}{\partial a}=-\frac{\partial y}{\partial a}$, and thus overall average bid for the high type decreases. In all other cases, the high type reduces her bid with $a$. Finally the bid of the low type is always lower than in the canonical model. This implies also that the seller's revenue decreases for all $a$.

\section{Fact 2}

$$
\frac{\partial \alpha}{\partial a}_{\mid a=1}=0
$$

\section{Proof of Fact 2:}

$$
\begin{aligned}
& \alpha=\operatorname{Pr}_{\left(\underline{\alpha}_{0}, \bar{\alpha}_{1}\right)}(l \mid l)=\alpha_{0}^{h}+\alpha_{1}^{h}=a(1-q)^{2}+\frac{1}{a}(1-q)^{2}+2 q-1 \\
& \left.\frac{\partial \alpha}{\partial a}\right|_{a=1}=(1-q)^{2} \frac{\partial\left(a+\frac{1}{a}\right)}{\partial a}_{\mid a=1}=(1-q)^{2}\left(1-\frac{1}{a^{2}}\right)_{\mid a=1}=0 .
\end{aligned}
$$

Fact 3 The bid of the low type is decreasing in $a$.

Proof of Fact 3: Follows from Fact 1.

Fact 4 (i)

$$
E\left[b_{a}(h)\right]=x\left(1+\frac{1-\alpha}{\alpha} \ln (1-\alpha)\right)-y \frac{1-\alpha}{\alpha} \ln (1-\alpha) .
$$

(ii) At $a=1$, the expected bid of the high type decreases in $a$ for low $q$ and increases in $a$ for high $q$.

\section{Proof of Fact 4:}


(i) Note that $\int \frac{b}{(x-b)^{2}} d b=\frac{1}{x-b}(x-b \ln (x-b)+x \ln (x-b))=\frac{x-(b-x) \ln (x-b)}{x-b}$, therefore, $\int_{y}^{\alpha x+(1-\alpha) y} \frac{b}{(b-x)^{2}} d b=\frac{\alpha x}{(1-\alpha)(x-y)}+\ln (1-\alpha)$.

Hence

$E\left[b_{a}(h)\right]=\frac{1-\alpha}{\alpha}(x-y) \int_{y}^{\alpha x+(1-\alpha) y} \frac{b}{(b-x)^{2}} d b=x\left(1+\frac{1-\alpha}{\alpha} \ln (1-\alpha)\right)-y \frac{1-\alpha}{\alpha} \ln (1-\alpha)$.

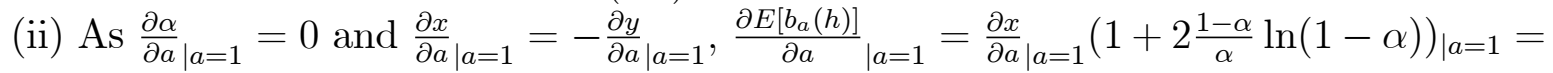
$\frac{\partial x}{\partial a}_{\mid a=1}\left(1+2 \frac{1-2(1-q)^{2}-2 q+1}{2(1-q)^{2}+2 q-1} \ln \left(1-2(1-q)^{2}-2 q+1\right)\right)$.

For $q>\frac{1}{2}$, the expression $\left(1+2 \frac{1-2(1-q)^{2}-2 q+1}{2(1-q)^{2}+2 q-1} \ln \left(1-2(1-q)^{2}-2 q+1\right)\right)$ is strictly increasing, negative for $q<q^{*}$ and positive for $q>q^{*}$ for some $q^{*} \in(0.5,1)$. As $\left.\frac{\partial x}{\partial a}\right|_{a=1}>0$, we are done.

Fact 5 (i)

$$
E\left[\max _{i=1,2} b_{a}^{i}(h)\right]=(x-y) 2\left(\left(\frac{1-\alpha}{\alpha}\right)^{2} \ln (1-\alpha)-\frac{1-\alpha}{\alpha}\right)+x
$$

(ii) The expectation of the maximal bid when both are high types decreases in $a$ for low $q$ and increases in $a$ for high $q$.

\section{Proof of Fact 5:}

(i) $\int \frac{b(b-y)}{(x-b)^{3}} d b=\frac{1}{2(x-b)^{2}}\left(2 b^{2} \ln (x-b)+2 x^{2} \ln (x-b)-4 b x+2 b y-x y+3 x^{2}-4 b x \ln (x-b)\right)=$ $-\ln (x-b)-\frac{-4 b x+2 b y-x y+3 x^{2}}{2(x-b)^{2}}$. Therefore, $\int_{y}^{\alpha x+(1-\alpha) y} \frac{b(b-y)}{(x-b)^{3}} d b=-\ln (1-\alpha)-\frac{\alpha(2 x-2 y-3 x \alpha+2 y \alpha)}{2(1-\alpha)^{2}(x-y)}$.

Hence

$E\left[\max _{i=1,2} b_{a}^{i}(h)\right]=2\left(\frac{1-\alpha}{\alpha}\right)^{2}(x-y) \int_{y}^{\alpha x+(1-\alpha) y} \frac{b(b-y)}{(x-b)^{3}} d b=-2\left(\frac{1-\alpha}{\alpha}\right)^{2}(x-y)(\ln (1-\alpha)+$ $\left.\frac{\alpha(2 x-2 y-3 x \alpha+2 y \alpha)}{2(1-\alpha)^{2}(x-y)}\right)=-(x-y) 2\left(\left(\frac{1-\alpha}{\alpha}\right)^{2} \ln (1-\alpha)+\frac{1-\alpha}{\alpha}\right)+x$.

(ii) Recalling that $\frac{\partial x}{\partial a}=-\frac{\partial y}{\partial a}$ and that $\frac{\partial \alpha}{\partial a}=0$ we have, $\frac{\partial E\left[\max _{i=1,2} b_{a}^{i}(h)\right]}{\partial a}=\frac{\partial x}{\partial a}\left(-4\left(\frac{1-\alpha}{\alpha}\right)^{2} \ln (1-\alpha)-4 \frac{1-\alpha}{\alpha}+1\right)$, and ${\frac{\partial E\left[\max _{i=1,2} b_{a}^{i}(h)\right]}{\partial a}}_{\mid a=1}=\frac{\partial x}{\partial a}\left(-4\left(\frac{2 q(1-q)}{q^{2}+(1-q)^{2}}\right)^{2} \ln (1-\alpha)-4 \frac{2 q(1-q)}{q^{2}+(1-q)^{2}}+1\right)$

For the expression $\left(-4\left(\frac{2 q(1-q)}{q^{2}+(1-q)^{2}}\right)^{2} \ln (1-2 q(1-q))-4 \frac{2 q(1-q)}{q^{2}+(1-q)^{2}}+1\right)$ there is a $\bar{q} \in(0.5,1)$ such that the expression is negative for $q<\bar{q}$ and positive for $q>\bar{q}$. As $\frac{\partial x}{\partial a}>0$, we are done.

Given the above facts we can write the profit function as:

$$
\begin{aligned}
\Pi= & \frac{1}{2} \gamma y+(1-\gamma) x+\frac{1-\alpha}{\alpha}(x-y)(1-\gamma) \ln (1-\alpha) \\
& +-\frac{1-\alpha^{2}}{\alpha}(x-y) \gamma \ln (1-\alpha)-\frac{1}{\alpha} \gamma\left(\frac{2 x-2 y-3 x \alpha+2 y \alpha}{2}\right) \\
= & x\left(\frac{\gamma}{2}+\frac{\alpha-\gamma}{\alpha}\left(\frac{1-\alpha}{\alpha} \ln (1-\alpha)+1\right)\right)+y\left(1-\frac{\gamma}{2}-\frac{\alpha-\gamma}{\alpha}\left(\frac{1-\alpha}{\alpha} \ln (1-\alpha)+1\right)\right)
\end{aligned}
$$

Taking a derivative with respect to $a$, recalling that $\left.\frac{d \alpha}{d a}\right|_{a=1}=0$ and that $\frac{\partial x}{\partial a}=-\frac{\partial y}{\partial a}$ we 
get,

$$
\begin{aligned}
{\left.\frac{\partial \Pi}{\partial a}\right|_{\mid a=1}} & \frac{\partial x}{\partial a}\left(\gamma-1+2 \frac{\alpha-\gamma}{\alpha}\left(\frac{1-\alpha}{\alpha} \ln (1-\alpha)+1\right)\right) \\
& =\frac{\partial x}{\partial a}(\gamma-1)<0 .
\end{aligned}
$$

Seller's revenue in the second price auction and a comparison across auctions: We now consider the seller's revenue in the second price auction and show that the profits of the seller are higher in the first-price auction.

In the second price auction, the low type always bids $E_{\left(\bar{\alpha}_{0}, \underline{\alpha}_{1}\right)}[v \mid l, l]$, and the high type bids at most $E_{\left(\underline{\alpha}_{0}, \bar{\alpha}_{1}\right)}[v \mid h, h]$ (either $b_{a}(h)=E_{\left(\underline{\alpha}_{0}, \bar{\alpha}_{1}\right)}[v \mid h, h]$ for low $a$ and $q$, where $E_{\left(\underline{\alpha}_{0}, \bar{\alpha}_{1}\right)}[v \mid h, h]>E_{\left(\bar{\alpha}_{0} \bar{\alpha}_{1}\right)}[v \mid h, h]=b_{a}(h)$ for higher $q$ and $a$, or where $E_{\left(\underline{\alpha}_{0}, \bar{\alpha}_{1}\right)}[v \mid h, h]>$ $\left.2 E_{\left(\bar{\alpha}_{0}, \underline{\alpha}_{1}\right)}[v \mid l, l]=b_{a}(h)\right)$. Let $\bar{R}^{S P A}$ be the revenue from a virtual auction where the low type bids $E_{\left(\bar{\alpha}_{0}, \underline{\alpha}_{1}\right)}[v \mid l, l]$ and the high type bids $E_{\left(\underline{\alpha}_{0}, \bar{\alpha}_{1}\right)}[v \mid h, h]$. Then the actual revenue in the second price auction must be weakly less than $\bar{R}^{S P A}$.

The seller's revenue in the second price auction satisfies:

$$
R^{S P A} \leq \bar{R}^{S P A}=x\left(\frac{\gamma}{2}\right)+y\left(1-\frac{\gamma}{2}\right)
$$

where $x=E_{\left(\underline{\alpha}_{0}, \bar{\alpha}_{1}\right)}[v \mid h, h], y=E_{\left(\bar{\alpha}_{0}, \underline{\alpha}_{1}\right)}[v \mid l, l]$, and $\gamma=\operatorname{Pr}(l \mid l)$. The seller's revenue in the first price auction is:

$R^{F P A}=x\left[\frac{\gamma}{2}+\frac{\alpha-\gamma}{\alpha}\left(\frac{1-\alpha}{\alpha} \ln (1-\alpha)+1\right)\right]+y\left[1-\frac{\gamma}{2}-\frac{\alpha-\gamma}{\alpha}\left(\frac{1-\alpha}{\alpha} \ln (1-\alpha)+1\right)\right]$

where $\alpha=\operatorname{Pr}\left(l \mid l,\left(\underline{\alpha}_{0}, \bar{\alpha}_{1}\right)\right)$. Thus, the difference in revenue between the two auctions is:

$$
R^{F P A}-R^{S P A} \geq R^{F P A}-\bar{R}^{S P A}=(x-y)\left[\frac{\alpha-\gamma}{\alpha}\left(\frac{1-\alpha}{\alpha} \ln (1-\alpha)+1\right)\right]>0 .
$$

Finally, to see that $R^{S P A}$ is decreasing in $a$, first note that when $a$ and $q$ are low:

$$
{\frac{\partial R_{1}^{S P A}}{\partial a}}_{\mid a=1}=\frac{\partial x}{\partial a}\left(\frac{\gamma}{2}\right)+\frac{\partial y}{\partial a}\left(1-\frac{\gamma}{2}\right)=-(1-\gamma) \frac{\partial x}{\partial a}<0
$$

Let $x^{\prime}=E_{\left(\bar{\alpha}_{0} \bar{\alpha}_{1}\right)}[v \mid h, h]$. Then the revenue when $q$ is high and $a$ is high:

$$
R_{2}^{S P A}=x^{\prime}\left(\frac{\gamma}{2}\right)+y\left(1-\frac{\gamma}{2}\right)
$$

Therefore: 


$$
{\left.\frac{\partial R_{2}^{S P A}}{\partial a}\right|_{\mid a=1}}^{S}=\frac{\partial x^{\prime}}{\partial a}\left(\frac{\gamma}{2}\right)+\frac{\partial y}{\partial a}\left(1-\frac{\gamma}{2}\right)<0
$$

since $\frac{\partial x^{\prime}}{\partial a}<0$. Finally in the last case: $\left.\frac{\partial R_{3}^{S P A}}{\partial a}\right|_{a=1}=\frac{\partial y}{\partial a}\left(1+\frac{\gamma}{2}\right)<0$.

\subsection{Proofs for Section 4}

Proposition 4A. Private value case: If $L<\frac{1}{1+a},(x, t)$ is an optimal mechanism if and only if it satisfies:

- $x_{i}(l, l)=x_{i}(l, h)=t_{i}(l, l)=t_{i}(l, h)=0$

- $x_{i}(h, l)=1, \sum_{i=1}^{2} x_{i}(h, h)=1$

- $t_{i}(h, l)=1$

- $t_{i}(h, h)=x_{i}(h, h)$

That is, if both buyers have the low valuation, then the seller does not allocate the object. If a single buyer has the high valuation, then she gets the object with probability 1. If both buyers have the high valuation, the seller can allocate to either buyer. Transfers are such that both buyers are fully insured against ambiguity and receive zero rents.

If $L>\frac{1}{1+a},(x, t)$ is an optimal mechanism if and only if it satisfies:

- $x_{i}(l, h)=t_{i}(l, h)=0$

- $\sum_{i=1}^{2} x_{i}(l, l)=1$

- $t_{i}(l, l)=L x_{i}(l, l)$

- $x_{i}(h, l)=1, \sum_{i=1}^{2} x_{i}(h, h)=1$

- $t_{i}(h, h)=x_{i}(h, h)-\frac{1}{2 a}(1-L) x_{i}(l, l)$

- $t_{i}(h, l)=1-\frac{1}{2 a}(1-L) x_{i}(l, l)$

That is, the seller allocates the object with probability 1. If the buyers have different valuations, the seller allocates to the one with the high valuation. Otherwise the seller may allocate to either buyer. Low types receives zero rents. Transfers for high types are pinned down by the binding incentive constraint and the full insurance condition.

The revenue of the seller is:

$$
\frac{1}{4} \sum_{i=1}^{2} t_{i}(l, l)+t_{i}(l, h)+t_{i}(h, l)+t_{i}(h, h)= \begin{cases}\frac{3}{4} & \text { if } L<\frac{1}{1+a} \\ \frac{1}{2}+\frac{L}{2}+\frac{(a-1)(1-L)}{4 a} & \text { if } L \geq \frac{1}{1+a}\end{cases}
$$


which is increasing in ambiguity. As a $\rightarrow \infty$, the revenue converges to $\frac{3}{4}+\frac{L}{4}$, which is full surplus.

Proof of Proposition 4A. First, we argue that any optimal mechanism must be a full insurance mechanism. Define a full insurance mechanism as one in which the ex post payoff of each buyer is independent of the type of her opponent:

Definition. The mechanism $(x, t)$ is a full insurance mechanism if for each player $i$ and for each $s, s^{\prime} \in\{l, h\}: v^{s} x_{i}(s, s)-t_{i}(s, s)=v^{s} x_{i}\left(s, s^{\prime}\right)-t_{i}\left(s, s^{\prime}\right)$.

Suppose that $(x, t)$ is an optimal mechanism, but $v^{s} x_{1}(s, s)-t_{1}(s, s)>v^{s} x_{1}\left(s, s^{\prime}\right)-$ $t_{1}\left(s, s^{\prime}\right)$. Then the equilibrium belief for type $s$ of player 1 is that her opponent is type $s$ with probability $\frac{1}{2 a}$ and type $s^{\prime}$ with probability $1-\frac{1}{2 a}$. Consider the alternative mechanism $\left(x^{\prime}, t^{\prime}\right)$ where:

- $t_{1}^{\prime}(s, s)=t_{1}(s, s)+\delta$

- $t_{1}^{\prime}\left(s, s^{\prime}\right)=t_{1}\left(s, s^{\prime}\right)-\frac{1}{2 a-1} \delta$

- $x_{1}^{\prime}\left(s^{\prime}, \cdot\right)=x_{1}\left(s^{\prime}, \cdot\right)$ and $t_{1}^{\prime}\left(s^{\prime}, \cdot\right)=t_{1}\left(s^{\prime}, \cdot\right)$

- $x_{2}^{\prime}(\cdot, \cdot)=x_{2}(\cdot, \cdot)$ and $t_{2}^{\prime}(\cdot, \cdot)=t_{2}(\cdot, \cdot)$

For sufficiently small $\delta$ :

- Type $s$ of player 1 gets the same utility in $\left(x^{\prime}, t^{\prime}\right)$ and $(x, t)$ for every report

- Type $s^{\prime}$ of player 1 gets the same utility in $\left(x^{\prime}, t^{\prime}\right)$ and $(x, t)$ from reporting $s^{\prime}$

- Type $s^{\prime}$ of player 1 gets weakly lower utility in $\left(x^{\prime}, t^{\prime}\right)$ than in $(x, t)$ from reporting $s$

- Both types of player 2 get the same utility in $\left(x^{\prime}, t^{\prime}\right)$ and $(x, t)$ for every report

- The seller gets a strictly higher revenue in $\left(x^{\prime}, t^{\prime}\right)$ than in $(x, t)$

Thus, the new mechanism is incentive compatible, individually rational, and yields strictly higher revenue to the seller.

Next, note that $x_{i}(l, h)=0$. For example, suppose that $x_{1}(l, h)>0$. Consider the alternative mechanism $\left(x^{\prime}, t^{\prime}\right)$ that differs from $(x, t)$ in the following way:

- $x_{1}^{\prime}(l, h)=x_{1}(l, h)-\delta$

- $t_{1}^{\prime}(l, h)=t_{1}(l, h)-\delta L$ 
- $x_{2}^{\prime}(h, l)=x_{2}(h, l)+\delta$

- $t_{2}^{\prime}(h, l)=t_{2}(h, l)+\delta$

Note that $\left(x^{\prime}, t^{\prime}\right)$ is incentive compatible, individually rational, and yields strictly higher revenue to the seller.

We can ignore the participation constraint of the high type as it is implied by the incentive constraint. Standard arguments imply that the participation constraint of the low type is binding. Lemma 1 then implies that the transfers for the low type satisfy:

$$
\begin{aligned}
t_{i}(l, l) & =L x_{i}(l, l) \\
t_{i}(l, h) & =0
\end{aligned}
$$

As usual, we ignore the incentive constraint of the low type and check ex post that it is satisfied. Then the incentive constraint of the high type must bind:

$$
\min _{p \in \Pi} p\left[x_{i}(h, h)-t_{i}(h, h)\right]+(1-p)\left[x_{i}(h, l)-t_{i}(h, l)\right]=\min _{p \in \Pi}(1-p)\left[x_{i}(l, l)-t_{i}(l, l)\right]
$$

Since $t_{i}(l, l)=L x_{i}(l, l), \min _{p \in \Pi}(1-p)\left[x_{i}(l, l)-t_{i}(l, l)\right]=\frac{1}{2 a}(1-L) x_{i}(l, l)$. Lemma 1 implies that $x_{i}(h, h)-t_{i}(h, h)=x_{i}(h, l)-t_{i}(h, l)$, so we have:

$$
\begin{aligned}
t_{i}(h, h) & =x_{i}(h, h)-\frac{1}{2 a}(1-L) x_{i}(l, l) \\
t_{i}(h, l) & =x_{i}(h, l)-\frac{1}{2 a}(1-L) x_{i}(l, l)
\end{aligned}
$$

Thus, we can write the seller's problem as:

$$
\max _{x_{i}, t_{i}} \sum_{i=1}^{2} L x_{i}(l, l)+x_{i}(h, h)+x_{i}(h, l)-\frac{1}{a}(1-L) x_{i}(l, l)
$$

Clearly, it is optimal to set $x_{i}(h, l)=1, \sum_{i=1}^{2} x_{i}(h, h)=1$, and $\sum_{i=1}^{2} x_{i}(l, l)$ to be either 1 or 0 , depending on the sign of $L-\frac{1}{a}(1-L)$.

Therefore, $(x, t)$ solves the seller's problem if and only if the allocation rule satisfies:

$$
\begin{aligned}
x_{i}(l, h) & =0 \\
x_{i}(h, l) & =1 \\
\sum_{i=1}^{2} x_{i}(h, h) & =1 \\
\sum_{i=1}^{2} x_{i}(l, l) & = \begin{cases}0 & \text { if } L<\frac{1}{1+a} \\
\text { any } w \in[0,1] & \text { if } L=\frac{1}{1+a} \\
1 & \text { if } L>\frac{1}{1+a}\end{cases}
\end{aligned}
$$


and transfers are given by equations 23,4 , and 5 .

Proposition 5A. Common value case:

(i) When $q \leq q^{*}(a)$, an optimal mechanism is, for $i \in\{1,2\}$ :

- $x_{i}(l, l)=x_{i}(h, h)=x_{i}(l, h)=x_{i}(h, l)=\frac{1}{2}$

- $t_{i}(h, l)=t_{i}(h, h)=t_{i}(l, h)=t_{i}(l, l)=\frac{1}{2}(1-q)$.

and the revenue of the seller is $1-q$.

(ii) When $q^{*}(a)<q<q^{* *}(a)$, an optimal mechanism is:

- $x_{i}(l, l)=x_{i}(h, h)=\frac{1}{2}, x_{i}(l, h)=0$, and $x_{i}(h, l)=1$

- $t_{i}(l, l)=t_{i}(l, h)=\frac{1}{2} \underline{\alpha}_{1}$

- $t_{i}(h, h)=\frac{q+\underline{\alpha}_{0}+\underline{\alpha}_{1}+\bar{\alpha}_{1}-1}{2}$

- $t_{i}(h, l)=\frac{q+\underline{\alpha}_{0}+\underline{\alpha}_{1}+\bar{\alpha}_{1}}{2}$

and the revenue of the seller is $\frac{1}{2}\left(\underline{\alpha}_{0}+2 \underline{\alpha}_{1}+\bar{\alpha}_{1}+q-q^{2}-(1-q)^{2}\right)$.

(iii) When $q \geq q^{* *}(a)$, an optimal mechanism is:

- $x_{i}(l, l)=x_{i}(h, h)=\frac{1}{2}, x_{i}(l, h)=0$, and $x_{i}(h, l)=1$

- $t_{i}(h, l)=\frac{1}{2}\left(1+\underline{\alpha}_{0}\right)$

- $t_{i}(h, h)=\frac{\underline{\alpha}_{0}}{2}$

- $t_{i}(l, h)=\frac{\underline{\alpha}_{1}}{2}+\left(\underline{\alpha}_{0}+\underline{\alpha}_{1}\right) \frac{1-q-\bar{\alpha}_{1}-\underline{\alpha}_{1}}{2\left(\bar{\alpha}_{0}+\bar{\alpha}_{1}+\underline{\alpha}_{0}+\underline{\alpha}_{1}-1\right)}$

- $t_{i}(l, l)=\frac{\underline{\alpha}_{1}}{2}-\left(1-\underline{\alpha}_{0}-\underline{\alpha}_{1}\right) \frac{1-q-\bar{\alpha}_{1}-\underline{\alpha}_{1}}{2\left(\bar{\alpha}_{0}+\bar{\alpha}_{1}+\underline{\alpha}_{0}+\underline{\alpha}_{1}-1\right)}$

and the revenue of the seller is $\frac{1}{2}\left(1-\left(q^{2}+(1-q)^{2}-\underline{\alpha}_{0}-\underline{\alpha}_{1}\right) \frac{\underline{\alpha}_{0}+\bar{\alpha}_{0}-q}{\bar{\alpha}_{0}+\bar{\alpha}_{1}+\underline{\alpha}_{0}+\underline{\alpha}_{1}-1}\right)$. 
Proof of Proposition 5A: The expressions for $q^{*}(a)$ and $q^{* *}(a)$ are as follows:

$$
\begin{aligned}
& q^{*}(a)= \begin{cases}\frac{1}{2}\left(\frac{6-7 a+2 a^{2}}{3-2 a+a^{2}}+\sqrt{\frac{-12 a+17 a^{2}-4 a^{3}}{\left(3-2 a+a^{2}\right)^{2}}}\right) & 1<a \leq \frac{1}{2}(-1+\sqrt{13}) \\
\frac{1}{4}\left(\frac{-7+6 a}{-2+a}+\sqrt{\frac{1-12 a+12 a^{2}}{(-2+a)^{2}}}\right) & \frac{1}{2}(-1+\sqrt{13})<a<2 \\
\frac{3}{5} & a=2 \\
\frac{1}{4}\left(\frac{-7+6 a}{-2+a}-\sqrt{\frac{1-12 a+12 a^{2}}{(-2+a)^{2}}}\right) & a>2\end{cases} \\
& q^{* *}(a)= \begin{cases}\frac{2-2 a+a^{2}}{2-a+a^{2}}+\frac{\sqrt{\frac{\left.-2 a+3 a^{2}-a^{3}\right)}{\left(2-a+a^{2}\right)^{2}}}}{\frac{\sqrt{2}}{2}\left(\frac{-5+3 a}{-3+a}+\sqrt{\frac{1-4 a+3 a^{2}}{(-3+a)^{2}}}\right)} & 1<a \leq \frac{1}{2}(-1+\sqrt{17}) \\
\frac{5}{8} & a=3 \\
\frac{1}{2}\left(\frac{-5+3 a}{-3+a}-\sqrt{\frac{1-4 a+3 a^{2}}{(-3+a)^{2}}}\right) & a>3\end{cases}
\end{aligned}
$$

We will ignore the incentive constraint of the low type and check ex post that it is satisfied. Therefore, the participation constraint of the low type must be binding.

Let $U_{i}^{h}$ be the utility of the high type in equilibrium and $U_{i}^{l}$ be the utility of the low type in equilibrium, that is:

$$
\begin{aligned}
U_{i}^{l} & \left.\equiv \min _{\alpha_{0}, \alpha_{1}} \alpha_{1} x_{i}(l, l)+\left(1-q-\alpha_{1}\right) x_{i}(l, h)-\left(\alpha_{0}+\alpha_{1}\right)\right) t_{i}(l, l)-\left(1-\alpha_{0}-\alpha_{1}\right) t_{i}(l, h) \\
U_{i}^{h} & \left.\equiv \min _{\alpha_{0}, \alpha_{1}}\left(1-q-\alpha_{1}\right) x_{i}(h, l)+\left(2 q-1+\alpha_{1}\right) x_{i}(h, h)-\left(1-\alpha_{0}-\alpha_{1}\right)\right) t_{i}(h, l)-\left(\alpha_{0}+\alpha_{1}\right) t_{i}(h, h)
\end{aligned}
$$

Note that it is optimal to set $U_{i}^{l}=0$. The incentive constraint of the high type is:

$$
\left.\min _{\alpha_{0}, \alpha_{1}}\left(1-q-\alpha_{1}\right) x_{i}(l, l)+\left(2 q-1+\alpha_{1}\right) x_{i}(l, h)-\left(\alpha_{0}+\alpha_{1}\right)\right) t_{i}(l, h)-\left(1-\alpha_{0}-\alpha_{1}\right) t_{i}(l, l) \leq U_{i}^{h}
$$

The participation constraint of the low type is:

$$
\left.\min _{\alpha_{0}, \alpha_{1}} \alpha_{1} x_{i}(l, l)+\left(1-q-\alpha_{1}\right) x_{i}(l, h)-\left(\alpha_{0}+\alpha_{1}\right)\right) t_{i}(l, l)-\left(1-\alpha_{0}-\alpha_{1}\right) t_{i}(l, h)=0
$$

We can subtract the latter from the former to get:

$$
\frac{\left(1-q-\underline{\alpha}_{1}-\bar{\alpha}_{1}\right) x_{i}(l, l)+\left(3 q-2+\underline{\alpha}_{1}+\bar{\alpha}_{1}\right) x_{i}(l, h)-U_{i}^{h}}{\underline{\alpha}_{0}+\underline{\alpha}_{1}+\bar{\alpha}_{0}+\bar{\alpha}_{1}-1} \leq t_{i}(l, h)-t_{i}(l, l)
$$

Define:

$$
\begin{aligned}
\Delta t_{l, i} & \equiv t_{i}(l, h)-t_{i}(l, l) \\
\Delta t_{h, i} & \equiv t_{i}(h, l)-t_{i}(h, h)
\end{aligned}
$$


We can write the expected transfers to the seller from each type as:

$$
\begin{aligned}
T_{i}^{l} & =\min _{\alpha_{0}, \alpha_{1}} \alpha_{1} x_{i}(l, l)+\left(1-q-\alpha_{1}\right) x_{i}(l, h)-\left(q^{2}+(1-q)^{2}-\left(\alpha_{0}+\alpha_{1}\right)\right) \Delta t_{l, i} \\
T_{i}^{h} & =\min _{\alpha_{0}, \alpha_{1}}\left(1-q-\alpha_{1}\right) x_{i}(h, l)+\left(2 q-1+\alpha_{1}\right) x_{i}(h, h)-\left(q^{2}+(1-q)^{2}-\left(\alpha_{0}+\alpha_{1}\right)\right) \Delta t_{h, i}-U_{i}^{h}
\end{aligned}
$$

Let $\alpha_{0}^{l}, \alpha_{1}^{l}$ and $\alpha_{0}^{h}, \alpha_{1}^{h}$ be solutions to these minimization problems. The seller chooses $x_{i}(l, l) \in\left[0, \frac{1}{2}\right], x_{i}(h, h) \in\left[0, \frac{1}{2}\right], x_{i}(l, h) \in[0,1], x_{i}(h, l) \in\left[0,1-x_{i}(l, h)\right], \Delta t_{l, i} \in R$, $\Delta t_{h, i} \in R$, and $U_{i}^{h} \geq 0$ to maximize $T_{i}^{l}+T_{i}^{h}$ subject to:

$$
\frac{\left(1-q-\underline{\alpha}_{1}-\bar{\alpha}_{1}\right) x_{i}(l, l)+\left(3 q-2+\underline{\alpha}_{1}+\bar{\alpha}_{1}\right) x_{i}(l, h)-U_{i}^{h}}{\underline{\alpha}_{0}+\underline{\alpha}_{1}+\bar{\alpha}_{0}+\bar{\alpha}_{1}-1} \leq \Delta t_{l, i}
$$

Clearly, it is optimal to set $x_{i}(h, h)=\frac{1}{2}$ and $x_{i}(h, l)=1-x_{i}(l, h)$. Thus, the seller's problem is:

$$
\begin{gathered}
\max _{x_{i}(l, l), x_{i}(l, h), \Delta t_{l, i}, \Delta t_{h, i}, U_{i}^{h}}\left\{\min _{\alpha_{0}, \alpha_{1}} \alpha_{1} x_{i}(l, l)+\left(1-q-\alpha_{1}\right) x_{i}(l, h)-\left(q^{2}+(1-q)^{2}-\left(\alpha_{0}+\alpha_{1}\right)\right) \Delta t_{l, i}\right. \\
\left.+\min _{\alpha_{0}, \alpha_{1}}\left(1-q-\alpha_{1}\right)\left(1-x_{i}(h, l)\right)+\frac{1}{2}\left(2 q-1+\alpha_{1}\right)-\left(q^{2}+(1-q)^{2}-\left(\alpha_{0}+\alpha_{1}\right)\right) \Delta t_{h, i}-U_{i}^{h}\right\}
\end{gathered}
$$

subject to:

$$
\begin{aligned}
\frac{\left(1-q-\underline{\alpha}_{1}-\bar{\alpha}_{1}\right) x_{i}(l, l)+\left(3 q-2+\underline{\alpha}_{1}+\bar{\alpha}_{1}\right) x_{i}(l, h)-U_{i}^{h}}{\underline{\alpha}_{0}+\underline{\alpha}_{1}+\bar{\alpha}_{0}+\bar{\alpha}_{1}-1} & \leq \Delta t_{l, i} \\
0 \leq x_{i}(l, l) & \leq \frac{1}{2} \\
0 \leq x_{i}(l, h) & \leq 1
\end{aligned}
$$

Define:

$$
\begin{aligned}
& \Delta t_{l, i}^{*}\left(x_{i}(l, l), x_{i}(l, h), U_{i}^{h}\right) \equiv \max \left\{0, \frac{\left(1-q-\underline{\alpha}_{1}-\bar{\alpha}_{1}\right) x_{i}(l, l)+\left(3 q-2+\underline{\alpha}_{1}+\bar{\alpha}_{1}\right) x_{i}(l, h)-U_{i}^{h}}{\underline{\alpha}_{0}+\underline{\alpha}_{1}+\bar{\alpha}_{0}+\bar{\alpha}_{1}-1},\right. \\
& \left.x_{i}(l, h)-x_{i}(l, l)\right\}
\end{aligned}
$$

We show that $\Delta t_{l, i}=\Delta t_{l, i}^{*}\left(x_{i}(l, l), x_{i}(l, h), U_{i}^{h}\right)$ is optimal. Note that $\Delta t_{l, i}<0$ implies $\alpha_{0}^{l}=\bar{\alpha}_{0}$, which implies that $q^{2}+(1-q)^{2}-\alpha_{0}^{l}-\alpha_{1}^{l}<0$, so it is profitable to increase $\Delta t_{l, i}$ (which also slackens $I C_{h}$ ). Similarly when $\Delta t_{l, i}<x_{i}(l, h)-x_{i}(l, l), \alpha_{1}^{l}=\bar{\alpha}_{1}$, which implies that $q^{2}+(1-q)^{2}-\alpha_{0}^{l}-\alpha_{1}^{l}<0$, so it is profitable to increase $\Delta t_{l, i}$. If $\Delta t_{l, i}>$ $x_{i}(l, h)-x_{i}(l, l) \geq 0$, then it is profitable to decrease $\Delta t_{l, i}$, which is possible if $\Delta t_{l, i}>$ $\frac{\left(1-q-\underline{\alpha}_{1}-\bar{\alpha}_{1}\right) x_{i}(l, l)+\left(3 q-2+\underline{\alpha}_{1}+\bar{\alpha}_{1}\right) x_{i}(l, h)-U_{i}^{h}}{\underline{\alpha}_{0}+\underline{\alpha}_{1}+\bar{\alpha}_{0}+\bar{\alpha}_{1}-1}$.

Define:

$$
\Delta t_{h, i}^{*}\left(x_{i}(l, h)\right) \equiv \max \left\{0, \frac{1}{2}-x_{i}(l, h)\right\}
$$


Similarly, it is optimal to set $\Delta t_{h, i}=\Delta t_{h, i}^{*}\left(x_{i}(l, h)\right)$. Thus, the problem becomes:

$$
\begin{aligned}
\max _{x_{i}(l, l), x_{i}(l, h), U_{i}^{h}} R\left(x_{i}(l, l), x_{i}(l, h), U_{i}^{h}\right) & =\min _{\alpha_{1}}\left\{\alpha_{1} x_{i}(l, l)+\left(1-q-\alpha_{1}\right) x_{i}(l, h)\right. \\
& \left.-\left(q^{2}+(1-q)^{2}-\left(\underline{\alpha}_{0}+\alpha_{1}\right)\right) \Delta t_{l, i}^{*}\left(x_{i}(l, l), x_{i}(l, h), U_{i}^{h}\right)\right\} \\
& +\min _{\alpha_{0}, \alpha_{1}}\left\{\left(1-q-\alpha_{1}\right)\left(1-x_{i}(l, h)\right)+\frac{2 q-1+\alpha_{1}}{2}\right. \\
& \left.-\left(q^{2}+(1-q)^{2}-\left(\alpha_{0}+\alpha_{1}\right)\right) \Delta t_{h, i}^{*}\left(x_{i}(l, h)\right)-U_{i}^{h}\right\}
\end{aligned}
$$

Now we show that $x_{i}(l, l)=\frac{1}{2}$ is optimal. Note that:

$$
\frac{\partial R}{\partial x_{i}(l, l)}=\left\{\begin{array}{ll}
\underline{\alpha}_{1}-\frac{\left(q^{2}+(1-q)^{2}-\underline{\alpha}_{0}-\underline{\alpha}_{1}\right)\left(1-q-\underline{\alpha}_{1}-\bar{\alpha}_{1}\right)}{\underline{\alpha}_{0}+\bar{\alpha}_{0}+\underline{\alpha}_{1}+\bar{\alpha}_{1}-1}>0 & x_{i}(l, l)>\max \left\{x_{i}(l, h), \frac{-x_{i}(l, h)\left(3 q-2+\underline{\alpha}_{1}+\bar{\alpha}_{1}\right)+U_{i}^{h}}{1-q-\underline{\alpha}_{1}-\bar{\alpha}_{1}}\right\} \\
\underline{\alpha}_{1}>0 & x_{i}(l, h)<x_{i}(l, l)<\frac{-x_{i}(l, h)\left(3 q-2+\underline{\alpha}_{1}+\bar{\alpha}_{1}\right)+U_{i}^{h}}{1-q-\underline{\alpha}_{1}-\bar{\alpha}_{1}} \\
q^{2}+(1-q)^{2}-\underline{\alpha}_{0}>0 & \frac{-x_{i}(l, h)\left(3 q-1-\underline{\alpha}_{0}-\bar{\alpha}_{0}\right)+U_{i}^{h}<x_{i}(l, l)<x_{i}(l, h)}{\underline{\alpha}_{0}+\bar{\alpha}_{0}-q} \\
\bar{\alpha}_{1}-\frac{\left(q^{2}+(1-q)^{2}-\underline{\alpha}_{0}-\bar{\alpha}_{1}\right)\left(1-q-\underline{\alpha}_{1}-\bar{\alpha}_{1}\right)}{\underline{\alpha}_{0}+\bar{\alpha}_{0}+\underline{\alpha}_{1}+\bar{\alpha}_{1}-1}>0 & x_{i}(l, l)<\min \left\{x_{i}(l, h), \frac{-x_{i}(l, h)\left(3 q-1-\underline{\alpha}_{0}-\bar{\alpha}_{0}\right)+U_{i}^{h}}{\underline{\alpha}_{0}+\bar{\alpha}_{0}-q}\right.
\end{array}\right\}
$$

Thus, the problem becomes:

$$
\begin{aligned}
\max _{x_{i}(l, h), U_{i}^{h}} R\left(x_{i}(l, h), U_{i}^{h}\right) & =\min _{\alpha_{1}}\left\{\frac{\alpha_{1}}{2}+\left(1-q-\alpha_{1}\right) x_{i}(l, h)\right. \\
& \left.-\left(q^{2}+(1-q)^{2}-\left(\underline{\alpha}_{0}+\alpha_{1}\right)\right) \Delta t_{l, i}^{*}\left(x_{i}(l, h), U_{i}^{h}\right)\right\} \\
& +\min _{\alpha_{1}}\left\{\left(1-q-\alpha_{1}\right)\left(1-x_{i}(l, h)\right)+\frac{2 q-1+\alpha_{1}}{2}\right. \\
& \left.-\left(q^{2}+(1-q)^{2}-\left(\underline{\alpha}_{0}+\alpha_{1}\right)\right) \Delta t_{h, i}^{*}\left(x_{i}(l, h)\right)-U_{i}^{h}\right\}
\end{aligned}
$$

Now we find the optimal $x_{i}(l, h)$ as a function of $U_{i}^{h}$ :

$$
\frac{\partial R\left(x_{i}(l, h), U_{i}^{h}\right)}{\partial x_{i}(l, h)}= \begin{cases}-\frac{\left(q^{2}+(1-q)^{2}-\underline{\alpha}_{0}-\underline{\alpha}_{1}\right)\left(3 q-2+\underline{\alpha}_{1}+\bar{\alpha}_{1}\right)}{\underline{\alpha}_{0}+\bar{\alpha}_{0}+\underline{\alpha}_{1}+\bar{\alpha}_{1}-1}<0 & x_{i}(l, h)>\max \left\{\frac{-\frac{1}{2}\left(\underline{\alpha}_{0}+\bar{\alpha}_{0}-q\right)+U_{i}^{h}}{3 q-1-\underline{\alpha}_{0}-\bar{\alpha}_{0}}, \frac{1}{2}\right\} \\ -\left(q^{2}+(1-q)^{2}-\underline{\alpha}_{0}-\underline{\alpha}_{1}\right)<0 & \frac{1}{2}<x_{i}(l, h)<\frac{-\frac{1}{2}\left(\underline{\alpha}_{0}+\bar{\alpha}_{0}-q\right)+U_{i}^{h}}{3 q-1-\underline{\alpha}_{0}-\bar{\alpha}_{0}} \\ -\frac{\left(q^{2}+(1-q)^{2}-\underline{\alpha}_{0}-\underline{\alpha}_{1}\right)\left(3 q-1-\underline{\alpha}_{0}-\bar{\alpha}_{0}\right)}{\underline{\alpha}_{0}+\overline{\bar{\alpha}}_{0}+\underline{\alpha}_{1}+\bar{\alpha}_{1}-1}<0 & \frac{-\frac{1}{2}\left(1-q-\underline{\alpha}_{1}-\bar{\alpha}_{1}\right)+U_{i}^{h}}{3 q-2+\underline{\alpha}_{1}+\bar{\alpha}_{1}}<x_{i}(l, h)<\frac{1}{2} \\ q^{2}+(1-q)^{2}-\underline{\alpha}_{0}-\underline{\alpha}_{1}>0 & x_{i}(l, h)<\min \left\{\frac{1}{2}, \frac{-\frac{1}{2}\left(1-q-\underline{\alpha}_{1}-\bar{\alpha}_{1}\right)+U_{i}^{h}}{3 q-2+\underline{\alpha}_{1}+\bar{\alpha}_{1}}\right\}\end{cases}
$$

Therefore:

$$
x_{i}^{*}(l, h)\left(U^{h}\right)= \begin{cases}0 & U_{i}^{h} \leq \frac{1}{2}\left(1-q-\underline{\alpha}_{1}-\bar{\alpha}_{1}\right) \\ \frac{-\frac{1}{2}\left(1-q-\underline{\alpha}_{1}-\bar{\alpha}_{1}\right)+U_{i}^{h}}{3 q-2+\underline{\alpha}_{1}+\bar{\alpha}_{1}} & \frac{1}{2}\left(1-q-\underline{\alpha}_{1}-\bar{\alpha}_{1}\right)<U_{i}^{h}<\frac{1}{2}(2 q-1) \\ \frac{1}{2} & U_{i}^{h} \geq \frac{1}{2}(2 q-1)\end{cases}
$$


We can now write the problem just in terms of $U_{i}^{h}$ :

$$
\begin{gathered}
\max _{U_{i}^{h}} R\left(U_{i}^{h}\right)=\frac{\underline{\alpha}_{1}}{2}+\left(1-q-\underline{\alpha}_{1}\right) x_{i}^{*}(l, h)\left(U_{i}^{h}\right)-\left(q^{2}+(1-q)^{2}-\left(\underline{\alpha}_{0}+\underline{\alpha}_{1}\right)\right) \Delta t_{l, i}^{*}\left(U_{i}^{h}\right) \\
+\left(1-q-\underline{\alpha}_{1}\right)\left(1-x_{i}^{*}(l, h)\left(U_{i}^{h}\right)\right)+\frac{2 q-1+\underline{\alpha}_{1}}{2} \\
-\left(q^{2}+(1-q)^{2}-\left(\underline{\alpha}_{0}+\underline{\alpha}_{1}\right)\right) \Delta t_{h, i}^{*}\left(x^{*}(l, h)\left(U_{i}^{h}\right)\right)-U_{i}^{h} \\
\frac{\partial R\left(U_{i}^{h}\right)}{\partial U_{i}^{h}}= \begin{cases}\frac{q^{2}+(1-q)^{2}-\underline{\alpha}_{0}-\underline{\alpha}_{1}}{\underline{\alpha}_{0}+\bar{\alpha}_{0}+\underline{\alpha}_{1}+\bar{\alpha}_{1}-1}-1 & U_{i}^{h}<\frac{1}{2}\left(1-q-\underline{\alpha}_{1}-\bar{\alpha}_{1}\right) \\
\frac{q^{2}+(1-q)^{2}-\underline{\alpha}_{0}-\underline{\alpha}_{1}}{3 q-2+\underline{\alpha}_{1}+\underline{\alpha}_{1}}-1 & \frac{1}{2}\left(1-q-\underline{\alpha}_{1}-\bar{\alpha}_{1}\right)<U_{i}^{h}<\frac{1}{2}(2 q-1) \\
-1 & U_{i}^{h}>\frac{1}{2}(2 q-1)\end{cases}
\end{gathered}
$$

Thus,

$$
U^{h}= \begin{cases}0 & q^{2}+(1-q)^{2}-\underline{\alpha}_{0}-\underline{\alpha}_{1} \leq \underline{\alpha}_{0}+\bar{\alpha}_{0}+\underline{\alpha}_{1}+\bar{\alpha}_{1}-1 \\ \frac{1}{2}\left(1-q-\underline{\alpha}_{1}-\bar{\alpha}_{1}\right) & \underline{\alpha}_{0}+\bar{\alpha}_{0}+\underline{\alpha}_{1}+\bar{\alpha}_{1}-1<q^{2}+(1-q)^{2}-\underline{\alpha}_{0}-\underline{\alpha}_{1}<3 q-2+\underline{\alpha}_{1}+\underline{\alpha}_{1} \\ \frac{1}{2}(2 q-1) & q^{2}+(1-q)^{2}-\underline{\alpha}_{0}-\underline{\alpha}_{1} \geq 3 q-2+\underline{\alpha}_{1}+\underline{\alpha}_{1}\end{cases}
$$

This is equivalent to:

$$
U^{h}= \begin{cases}0 & q \geq q^{* *}(a) \\ \frac{1}{2}\left(1-q-\underline{\alpha}_{1}-\bar{\alpha}_{1}\right) & q^{*}(a)<q<q^{* *}(a) \\ \frac{1}{2}(2 q-1) & q \leq q^{*}(a)\end{cases}
$$

Thus, in the optimal symmetric mechanism, $x_{i}(l, l)=x_{i}(h, h)=\frac{1}{2}$, and:

$$
\begin{aligned}
& x_{i}(l, h)= \begin{cases}0 & q \geq q^{* *}(a) \\
\frac{1}{2} & q^{*}(a)<q<q^{* *}(a) \\
\frac{1}{2} & q \leq q^{*}(a)\end{cases} \\
& \Delta t_{l, i}= \begin{cases}\frac{1-q-\underline{\alpha}_{1}-\bar{\alpha}_{1}}{2\left(\underline{\alpha}_{0}+\underline{\alpha}_{1}+\bar{\alpha}_{0}+\bar{\alpha}_{1}-1\right)} & q \geq q^{* *}(a) \\
0 & q^{*}(a)<q<q^{* *}(a) \\
0 & q \leq q^{*}(a)\end{cases} \\
& \Delta t_{h, i}= \begin{cases}\frac{1}{2} & q \geq q^{* *}(a) \\
\frac{1}{2} & q^{*}(a)<q<q^{* *}(a) \\
0 & q \leq q^{*}(a)\end{cases}
\end{aligned}
$$


To recover the transfers $t_{i}(l, l), t_{i}(l, h), t_{i}(h, l)$, and $t_{i}(h, h)$, use:

$$
\begin{aligned}
& \left.\min _{\alpha_{0}, \alpha_{1}} \alpha_{1} x_{i}(l, l)+\left(1-q-\alpha_{1}\right) x_{i}(l, h)-\left(\alpha_{0}+\alpha_{1}\right)\right) t_{i}(l, l)-\left(1-\alpha_{0}-\alpha_{1}\right)\left(t_{i}(l, l)+\Delta t_{l, i}\right)=0 \\
& \min _{\alpha_{0}, \alpha_{1}}\left\{\left(1-q-\alpha_{1}\right) x_{i}(h, l)+\left(2 q-1+\alpha_{1}\right) x_{i}(h, h)\right. \\
& \left.\left.-\left(1-\alpha_{0}-\alpha_{1}\right)\right)\left(t_{i}(h, h)+\Delta t_{h, i}\right)-\left(\alpha_{0}+\alpha_{1}\right) t_{i}(h, h)\right\}=U_{i}^{h}
\end{aligned}
$$

Now we show that the optimal symmetric mechanism is fully optimal. Suppose that there exists an asymmetric mechanism $(x, t)$ that is optimal. Define:

$$
\begin{aligned}
\bar{x}(\cdot, \cdot) & \equiv \frac{1}{2} x_{1}(\cdot, \cdot)+\frac{1}{2} x_{2}(\cdot, \cdot) \\
\bar{t}(\cdot, \cdot) & \equiv \frac{1}{2} t_{1}(\cdot, \cdot)+\frac{1}{2} t_{2}(\cdot, \cdot)
\end{aligned}
$$

Consider the following symmetric mechanism:

$$
\begin{aligned}
x_{i}^{\prime}(\cdot, \cdot)=\bar{x}(\cdot, \cdot) & \\
t_{i}^{\prime}(l, \cdot)=\bar{t}(l, \cdot)+\min _{\alpha_{0}, \alpha_{1}} & \left\{\alpha_{1} \bar{x}(l, l)+\left(1-q-\alpha_{1}\right) \bar{x}(l, h)\right. \\
& \left.-\left(\alpha_{0}+\alpha_{1}\right) \bar{t}(l, l)-\left(1-\alpha_{0}-\alpha_{1}\right) \bar{t}(l, h)-\frac{1}{2} \sum_{i=1}^{2} U_{i}^{l}\right\} \\
t_{i}^{\prime}(h, \cdot)=\bar{t}(h, \cdot)+\min _{\alpha_{0}, \alpha_{1}} & \left\{\left(1-q-\alpha_{1}\right) \bar{x}(h, l)+\left(2 q-1+\alpha_{1}\right) \bar{x}(h, h)\right. \\
- & \left.\left(1-\alpha_{0}+\alpha_{1}\right) \bar{t}(h, l)-\left(\alpha_{0}+\alpha_{1}\right) \bar{t}(h, h)-\frac{1}{2} \sum_{i=1}^{2} U_{i}^{h}\right\}
\end{aligned}
$$

By construction, the high type gets $\frac{1}{2} \sum_{i=1}^{2} U_{i}^{h}$ in equilibrium and the low type gets $\frac{1}{2} \sum_{i=1}^{2} U_{i}^{l}$ in equilibrium; therefore both participation constraints are satisfied.

Define $\Delta \bar{x}_{l} \equiv \bar{x}(l, h)-\bar{x}(l, l), \Delta \bar{t}_{l} \equiv \bar{t}(l, h)-\bar{t}(l, l), \Delta x_{l, i} \equiv x_{i}(l, h)-x_{i}(l, l)$, and $\Delta t_{l, i} \equiv t_{i}(l, h)-t_{i}(l, l)$. To see that $I C_{h}$ is satisfied, first note that:

$$
\begin{aligned}
& \min _{\alpha_{0}, \alpha_{1}}\left(1-q-\alpha_{1}\right) \bar{x}(l, l)+\left(2 q-1+\alpha_{1}\right) \bar{x}(l, h)-\left(1-\alpha_{0}-\alpha_{1}\right) \bar{t}(l, l)-\left(\alpha_{0}+\alpha_{1}\right) \bar{t}(l, h) \\
& =\frac{1}{2} \sum_{i=1}^{2} \min _{\alpha_{0}, \alpha_{1}}\left(1-q-\alpha_{1}\right) x_{i}(l, l)+\left(2 q-1+\alpha_{1}\right) x_{i}(l, h)-\left(1-\alpha_{0}-\alpha_{1}\right) t_{i}(l, l)-\left(\alpha_{0}+\alpha_{1}\right) t_{i}(l, h) \\
& +\min _{\alpha_{0}, \alpha_{1}} \alpha_{1} \Delta \bar{x}_{l}-\left(\alpha_{0}+\alpha_{1}\right) \Delta \bar{t}_{l}-\frac{1}{2} \sum_{i=1}^{2} \min _{\alpha_{0}, \alpha_{1}} \alpha_{1} \Delta x_{l, i}-\left(\alpha_{0}+\alpha_{1}\right) \Delta t_{l, i}
\end{aligned}
$$

and by definition:

$\frac{1}{2} \sum_{i=1}^{2} \min _{\alpha_{0}, \alpha_{1}} \alpha_{1} x_{i}(l, l)+\left(1-q-\alpha_{1}\right) x_{i}(l, h)-\left(\alpha_{0}+\alpha_{1}\right) t_{i}(l, l)-\left(1-\alpha_{0}-\alpha_{1}\right) t_{i}(l, h)-U_{i}^{l}=0$ 


\section{Therefore:}

$$
\begin{aligned}
& \min _{\alpha_{0}, \alpha_{1}}\left(1-q-\alpha_{1}\right) \bar{x}(l, l)+\left(2 q-1+\alpha_{1}\right) \bar{x}(l, h)-\left(1-\alpha_{0}-\alpha_{1}\right) \bar{t}(l, l)-\left(\alpha_{0}+\alpha_{1}\right) \bar{t}(l, h) \\
& -\left(\min _{\alpha_{0}, \alpha_{1}} \alpha_{1} \bar{x}(l, l)+\left(1-q-\alpha_{1}\right) \bar{x}(l, h)-\left(\alpha_{0}+\alpha_{1}\right) \bar{t}(l, l)-\left(1-\alpha_{0}-\alpha_{1}\right) \bar{t}(l, h)\right)+\frac{1}{2} \sum_{i=1}^{2} U_{i}^{l} \\
& =\frac{1}{2} \sum_{i=1}^{2} \min _{\alpha_{0}, \alpha_{1}}\left(1-q-\alpha_{1}\right) x_{i}(l, l)+\left(2 q-1+\alpha_{1}\right) x_{i}(l, h)-\left(1-\alpha_{0}-\alpha_{1}\right) t_{i}(l, l)-\left(\alpha_{0}+\alpha_{1}\right) t_{i}(l, h) \\
& +\min _{\alpha_{0}, \alpha_{1}} \alpha_{1} \Delta \bar{x}_{l}-\left(\alpha_{0}+\alpha_{1}\right) \Delta \bar{t}_{l}-\frac{1}{2} \sum_{i=1}^{2} \min _{\alpha_{0}, \alpha_{1}} \alpha_{1} \Delta x_{l, i}-\left(\alpha_{0}+\alpha_{1}\right) \Delta t_{l, i} \\
& +\frac{1}{2} \sum_{i=1}^{2} \min _{\alpha_{0}, \alpha_{1}} \alpha_{1} x_{i}(l, l)+\left(1-q-\alpha_{1}\right) x_{i}(l, h)-\left(\alpha_{0}+\alpha_{1}\right) t_{i}(l, l)-\left(1-\alpha_{0}-\alpha_{1}\right) t_{i}(l, h)-U_{i}^{l} \\
& -\left(\min _{\alpha_{0}, \alpha_{1}} \alpha_{1} \bar{x}(l, l)+\left(1-q-\alpha_{1}\right) \bar{x}(l, h)-\left(\alpha_{0}+\alpha_{1}\right) \bar{t}(l, l)-\left(1-\alpha_{0}-\alpha_{1}\right) \bar{t}(l, h)\right)+\frac{1}{2} \sum_{i=1}^{2} U_{i}^{l} \\
& =\frac{1}{2} \sum_{i=1}^{2} \min _{\alpha_{0}, \alpha_{1}}\left(1-q-\alpha_{1}\right) x_{i}(l, l)+\left(2 q-1+\alpha_{1}\right) x_{i}(l, h)-\left(1-\alpha_{0}-\alpha_{1}\right) t_{i}(l, l)-\left(\alpha_{0}+\alpha_{1}\right) t_{i}(l, h) \\
& +\min _{\alpha_{0}, \alpha_{1}} \alpha_{1} \Delta \bar{x}_{l}-\left(\alpha_{0}+\alpha_{1}\right) \Delta \bar{t}_{l}-\frac{1}{2} \sum_{i=1}^{2} \min _{\alpha_{0}, \alpha_{1}} \alpha_{1} \Delta x_{l, i}-\left(\alpha_{0}+\alpha_{1}\right) \Delta t_{l, i} \\
& +\frac{1}{2} \sum_{i=1}^{2} \min _{\alpha_{0}, \alpha_{1}}-\alpha_{1} \Delta x_{l, i}+\left(\alpha_{0}+\alpha_{1}\right) \Delta t_{l, i}-\min _{\alpha_{0}, \alpha_{1}}-\alpha_{1} \Delta \bar{x}_{l}+\left(\alpha_{0}+\alpha_{1}\right) \Delta \bar{t}_{l} \leq \frac{1}{2} \sum_{i=1}^{2} U_{i}^{h}
\end{aligned}
$$

The last inequality follows because:

$$
\begin{aligned}
& \min _{\alpha_{0}, \alpha_{1}} \alpha_{1} \Delta \bar{x}_{l}-\left(\alpha_{0}+\alpha_{1}\right) \Delta \bar{t}_{l}-\frac{1}{2} \sum_{i=1}^{2} \min _{\alpha_{0}, \alpha_{1}} \alpha_{1} \Delta x_{l, i}-\left(\alpha_{0}+\alpha_{1}\right) \Delta t_{l, i} \\
& +\frac{1}{2} \sum_{i=1}^{2} \min _{\alpha_{0}, \alpha_{1}}-\alpha_{1} \Delta x_{l, i}+\left(\alpha_{0}+\alpha_{1}\right) \Delta t_{l, i}-\min _{\alpha_{0}, \alpha_{1}}-\alpha_{1} \Delta \bar{x}_{l}+\left(\alpha_{0}+\alpha_{1}\right) \Delta \bar{t}_{l} \\
& =\min _{\alpha_{0}, \alpha_{1}} \alpha_{1} \Delta \bar{x}_{l}-\left(\alpha_{0}+\alpha_{1}\right) \Delta \bar{t}_{l}-\frac{1}{2} \sum_{i=1}^{2} \min _{\alpha_{0}, \alpha_{1}} \alpha_{1} \Delta x_{l, i}-\left(\alpha_{0}+\alpha_{1}\right) \Delta t_{l, i} \\
& +\max _{\alpha_{0}, \alpha_{1}} \alpha_{1} \Delta \bar{x}_{l}-\left(\alpha_{0}+\alpha_{1}\right) \Delta \bar{t}_{l}-\frac{1}{2} \sum_{i=1}^{2} \max _{\alpha_{0}, \alpha_{1}} \alpha_{1} \Delta x_{l, i}-\left(\alpha_{0}+\alpha_{1}\right) \Delta t_{l, i} \\
& =\left(\underline{\alpha}_{1}+\bar{\alpha}_{1}\right) \Delta \bar{x}_{l}-\left(\underline{\alpha}_{0}+\bar{\alpha}_{0}+\underline{\alpha}_{1}+\bar{\alpha}_{1}\right) \Delta \bar{t}_{l}-\frac{1}{2} \sum_{i=1}^{2}\left(\underline{\alpha}_{1}+\bar{\alpha}_{1}\right) \Delta x_{l, i}-\left(\underline{\alpha}_{0}+\bar{\alpha}_{0}+\underline{\alpha}_{1}+\bar{\alpha}_{1}\right) \Delta t_{l, i}=0
\end{aligned}
$$


The proof that $I C_{l}$ is satisfied is analogous. Define $\Delta \bar{x}_{h} \equiv \bar{x}(h, l)-\bar{x}(h, h), \Delta \bar{t}_{h} \equiv$ $\bar{t}(h, l)-\bar{t}(h, h), \Delta x_{h, i} \equiv x_{i}(h, l)-x_{i}(h, h)$, and $\Delta t_{h, i} \equiv t_{i}(h, l)-t_{i}(h, h)$. Then:

$$
\begin{aligned}
& \min _{\alpha_{0}, \alpha_{1}} \alpha_{1} \bar{x}(h, l)+\left(1-q-\alpha_{1}\right) \bar{x}(h, h)-\left(\alpha_{0}+\alpha_{1}\right) \bar{t}(h, l)-\left(1-\alpha_{0}-\alpha_{1}\right) \bar{t}(h, h) \\
& -\left(\min _{\alpha_{0}, \alpha_{1}}\left(1-q-\alpha_{1}\right) \bar{x}(h, l)+\left(2 q-1+\alpha_{1}\right) \bar{x}(h, h)-\left(1-\alpha_{0}-\alpha_{1}\right) \bar{t}(h, l)-\left(\alpha_{0}+\alpha_{1}\right) \bar{t}(h, h)\right) \\
& +\frac{1}{2} \sum_{i=1}^{2} U_{i}^{h} \\
& =\frac{1}{2} \sum_{i=1}^{2} \min _{\alpha_{0}, \alpha_{1}} \alpha_{1} x_{i}(h, l)+\left(1-q-\alpha_{1}\right) x_{i}(h, h)-\left(\alpha_{0}+\alpha_{1}\right) t_{i}(h, l)-\left(1-\alpha_{0}-\alpha_{1}\right) t_{i}(h, h) \\
& +\min _{\alpha_{0}, \alpha_{1}} \alpha_{1} \Delta \bar{x}_{h}-\left(\alpha_{0}+\alpha_{1}\right) \Delta \bar{t}_{h}-\frac{1}{2} \sum_{i=1}^{2} \min _{\alpha_{0}, \alpha_{1}} \alpha_{1} \Delta x_{h, i}-\left(\alpha_{0}+\alpha_{1}\right) \Delta t_{h, i} \\
& +\frac{1}{2} \sum_{i=1}^{2}\left(\min _{\alpha_{0}, \alpha_{1}}\left(1-q-\alpha_{1}\right) x_{i}(h, l)+\left(2 q-1+\alpha_{1}\right) x_{i}(h, h)-\left(1-\alpha_{0}-\alpha_{1}\right) t_{i}(h, l)-\left(\alpha_{0}+\alpha_{1}\right) t_{i}(h, h)\right. \\
& \left.-U_{i}^{h}\right) \\
& -\left(\min _{\alpha_{0}, \alpha_{1}}\left(1-q-\alpha_{1}\right) \bar{x}(h, l)+\left(2 q-1+\alpha_{1}\right) \bar{x}(h, h)-\left(1-\alpha_{0}-\alpha_{1}\right) \bar{t}(h, l)-\left(\alpha_{0}+\alpha_{1}\right) \bar{t}(h, h)\right) \\
& +\frac{1}{2} \sum_{i=1}^{2} U_{i}^{h} \\
& =\frac{1}{2} \sum_{i=1}^{2} \min _{\alpha_{0}, \alpha_{1}} \alpha_{1} x_{i}(h, l)+\left(1-q-\alpha_{1}\right) x_{i}(h, h)-\left(\alpha_{0}+\alpha_{1}\right) t_{i}(h, l)-\left(1-\alpha_{0}-\alpha_{1}\right) t_{i}(h, h) \\
& +\min _{\alpha_{0}, \alpha_{1}} \alpha_{1} \Delta \bar{x}_{h}-\left(\alpha_{0}+\alpha_{1}\right) \Delta \bar{t}_{h}-\frac{1}{2} \sum_{i=1}^{2} \min _{\alpha_{0}, \alpha_{1}} \alpha_{1} \Delta x_{h, i}-\left(\alpha_{0}+\alpha_{1}\right) \Delta t_{h, i} \\
& +\frac{1}{2} \sum_{i=1}^{2} \min _{\alpha_{0}, \alpha_{1}}-\alpha_{1} \Delta x_{h, i}+\left(\alpha_{0}+\alpha_{1}\right) \Delta t_{h, i}-\min _{\alpha_{0}, \alpha_{1}}-\alpha_{1} \Delta \bar{x}_{h}+\left(\alpha_{0}+\alpha_{1}\right) \Delta \bar{t}_{h} \leq \frac{1}{2} \sum_{i=1}^{2} U_{i}^{l}
\end{aligned}
$$

Finally, note that $\frac{1}{2} \sum_{i=1}^{2} t_{i}^{\prime}(l, l) \geq \bar{t}(l, l), \frac{1}{2} \sum_{i=1}^{2} t_{i}^{\prime}(l, h) \geq \bar{t}(l, h), \frac{1}{2} \sum_{i=1}^{2} t_{i}^{\prime}(h, l) \geq$ $\bar{t}(h, l)$, and $\frac{1}{2} \sum_{i=1}^{2} t_{i}^{\prime}(h, h) \geq \bar{t}(h, h)$, so the symmetric mechanism $\left(x^{\prime}, t^{\prime}\right)$ is incentive compatible and yields weakly greater revenue to the seller than $(x, t)$.

\subsection{Proofs for Section 5}

\subsubsection{Many bidders}

Each of $n$ bidders has a common valuation $v \in\{0,1\}$ for an object, where $\operatorname{Pr}(v=0)=\frac{1}{2}$. A public signal $s^{*} \in\{l, h\}$ is drawn so that $\operatorname{Pr}\left(s^{*}=h \mid v=1\right)=\operatorname{Pr}\left(s^{*}=l \mid v=0\right)=q>\frac{1}{2}$. 
With probability $\alpha_{v}$, all bidders receive the signal $s^{*}$ in state $v$ : that is, with probability $\alpha_{v}$ each bidder's signal is $s_{i}=s^{*}$. Otherwise each bidder's signal is drawn independently from the same distribution as the public signal so that $\operatorname{Pr}\left(s_{i}=h \mid v=1\right)=\operatorname{Pr}\left(s_{i}=l \mid v=\right.$ $0)=q>\frac{1}{2}$. The bidders consider as possible the following values of $\alpha_{v}$ :

$$
\begin{aligned}
& 0 \leq \alpha_{0} \leq a \\
& 0 \leq \alpha_{1} \leq a
\end{aligned}
$$

Let $\bar{\alpha}_{0}=\bar{\alpha}_{1} \equiv a$. Define $P\left(k l, n-k-1 h \mid h,\left(\alpha_{0}, \alpha_{1}\right)\right)$ as the probability of $k l$ signals (ordered), and $n-k-1 h$ signals, conditional on an $h$ signal under beliefs $\left(\alpha_{0}, \alpha_{1}\right)$, and let $E_{\left(\alpha_{0}, \alpha_{1}\right)}[v \mid k l, n-k h]$ be the corresponding conditional expectation. That is:

$$
\begin{aligned}
P\left(k l, n-k-1 h \mid h,\left(\alpha_{0}, \alpha_{1}\right)\right) & =(1-q)\left(\left(1-\alpha_{0}\right) q^{k}(1-q)^{n-k-1}+\alpha_{0} \mathcal{I}(k=0)\right) \\
& +q\left(\left(1-\alpha_{1}\right) q^{n-k-1}(1-q)^{k}+\alpha_{1} \mathcal{I}(k=0)\right)
\end{aligned}
$$

and $E_{\left(\alpha_{0}, \alpha_{1}\right)}[v \mid k l, n-k h]=\frac{q\left(\left(1-\alpha_{1}\right) q^{n-k-1}(1-q)^{k}+\alpha_{1} \mathcal{I}(k=0)\right)}{(1-q)\left(\left(1-\alpha_{0}\right) q^{k}(1-q)^{n-k-1}+\alpha_{0} \mathcal{I}(k=0)\right)+q\left(\left(1-\alpha_{1}\right) q^{n-k-1}(1-q)^{k}+\alpha_{1} \mathcal{I}(k=0)\right)}$

Proposition 6A: When ambiguity is not too large, there exists a symmetric equilibrium of the first price auction where for sufficiently large $n$ :

- The low type has beliefs $\left(\alpha_{0}, \alpha_{1}\right)=\left(a_{0}, 0\right)$ and bids $b_{a}(l)=E_{\left(\bar{\alpha}_{0}, 0\right)}[v \mid l, \ldots, l]$.

- $\quad$ The high type has beliefs $\left(\alpha_{0}, \alpha_{1}\right)=(0, a)$ and mixes on the support $\left[b_{a}(l), \bar{b}_{F}\right]$ according to distribution $F(b)$, where:

$$
\bar{b}_{F}=\sum_{k=0}^{n-2}\left(\begin{array}{c}
n-1 \\
k
\end{array}\right) P(k l, n-k-1 h \mid h,(0, a)) E_{\left(0, \bar{\alpha}_{1}\right)}[v \mid k l, n-k h]+P(n-1 l \mid h,(0, a)) b_{a}(l)
$$

and for $b \in\left[b_{a}(l), \bar{b}_{F}\right], F(b)$ solves:

$$
\begin{aligned}
& \sum_{k=0}^{n-2}\left(\begin{array}{c}
n-1 \\
k
\end{array}\right) P(k l, n-k-1 h \mid h,(0, a))\left(E_{\left(0, \bar{\alpha}_{1}\right)}[v \mid k l, n-k h]-b\right) F(b)^{n-k-1} \\
& =P(n-1 l \mid h,(0, a))\left(b-b_{a}(l)\right)
\end{aligned}
$$

Proof. Note that $\operatorname{Pr}(l, \ldots, l \mid l)=(1-q)\left(\alpha_{1}+\left(1-\alpha_{1}\right)(1-q)^{n-1}\right)+q\left(\alpha_{0}+\left(1-\alpha_{0}\right) q^{n-1}\right)$, and $E(v \mid l, \ldots, l)=\frac{(1-q)\left(\alpha_{1}+\left(1-\alpha_{1}\right)(1-q)^{n-1}\right)}{(1-q)\left(\alpha_{1}+\left(1-\alpha_{1}\right)(1-q)^{n-1}\right)+q\left(\alpha_{0}+\left(1-\alpha_{0}\right) q^{n-1}\right)}$. Thus, the equilibrium utility of the low type is:

$$
\min _{\alpha_{0}, \alpha_{1}}(1-q)\left(\alpha_{1}+\left(1-\alpha_{1}\right)(1-q)^{n-1}\right)(1-b)-q\left(\alpha_{0}+\left(1-\alpha_{0}\right) q^{n-1}\right) b
$$


Note that the derivative with respect to $\alpha_{0}$ is $-q\left(1-q^{n-1}\right)<0$ and the derivative with respect to $\alpha_{1}$ is $(1-q)\left(1-(1-q)^{n-1}\right)>0$. Thus, in equilibrium, the low type's beliefs are given by $\left(\alpha_{0}, \alpha_{1}\right)=\left(\bar{\alpha}_{0}, 0\right)$. Under the equilibrium bid, the low type gets zero utility. Note that any bid below $b_{a}(l)$ yields zero utility, and any bid above $b_{a}(l)$ yields negative utility under the equilibrium beliefs (for sufficiently small $a$ ). Therefore any deviation is also not profitable evaluated under the utility minimising beliefs. The equilibrium utility of the high type (under no 'no hedging') is:

$$
\min _{\alpha_{0}, \alpha_{1}} \sum_{k=0}^{n-1} \frac{1}{n-k}\left(\begin{array}{c}
n-1 \\
k
\end{array}\right) P\left(k l, n-k-1 h \mid h,\left(\alpha_{0}, \alpha_{1}\right)\right)\left[E_{\left(\alpha_{0}, \alpha_{1}\right)}[v \mid k l, n-k h]-\left(\int_{b_{a}(l)}^{\bar{b}_{F}} b d F^{n-k}(b)\right)\right]
$$

The derivative of the utility with respect to $\alpha_{0}$ is:

$$
\begin{aligned}
& \sum_{k=0}^{n-1} \frac{1}{n-k}\left(\begin{array}{c}
n-1 \\
k
\end{array}\right)\left[-\frac{\partial P\left(k l, n-k-1 h \mid h,\left(\alpha_{0}, \alpha_{1}\right)\right)}{\partial \alpha_{0}}\left(\int_{b_{a}(l)}^{\bar{b}_{F}} b d F^{n-k}(b)\right)\right] \\
& =\sum_{k=0}^{n-1} \frac{1}{n-k}\left(\begin{array}{c}
n-1 \\
k
\end{array}\right)\left[-(1-q)\left(-(1-q)^{n-k-1} q^{k}+\mathcal{I}(k=0)\right)\left(\int_{b_{a}(l)}^{\bar{b}_{F}} b d F^{n-k}(b)\right)\right] \\
& =\sum_{k=1}^{n-1} \frac{1}{n-k}\left(\begin{array}{c}
n-1 \\
k
\end{array}\right)\left[(1-q)^{n-k} q^{k}\left(\int_{b_{a}(l)}^{\bar{b}_{F}} b d F^{n-k}(b)\right)\right]-\frac{1}{n}(1-q)\left(1-(1-q)^{n-1}\right)\left(\int_{b_{a}(l)}^{\bar{b}_{F}} b d F^{n}(b)\right) .
\end{aligned}
$$

In Lemma $6 \mathrm{~A}$ below we show that the above derivative is positive. The derivative of the utility with respect to $\alpha_{1}$ is:

$$
\begin{aligned}
& =\min _{\alpha_{0}, \alpha_{1}} \sum_{k=0}^{n-1} \frac{1}{n-k}\left(\begin{array}{c}
n-1 \\
k
\end{array}\right)\left[\frac{\partial P\left(k l, n-k-1 h \mid h,\left(\alpha_{0}, \alpha_{1}\right)\right) E_{\left(\alpha_{0}, \alpha_{1}\right)}[v \mid k l, n-k h]}{\partial \alpha_{1}}\right. \\
& \left.-\frac{\partial P\left(k l, n-k-1 h \mid h,\left(\alpha_{0}, \alpha_{1}\right)\right)}{\partial \alpha_{1}}\left(\int_{b_{a}(l)}^{\bar{b}_{F}} b d F^{n-k}(b)\right)\right] \\
& =\sum_{k=0}^{n-1} \frac{1}{n-k}\left(\begin{array}{c}
n-1 \\
k
\end{array}\right)\left[\frac{\partial P\left(k l, n-k-1 h \mid h,\left(\alpha_{0}, \alpha_{1}\right)\right)}{\partial \alpha_{1}}\left(1-\int_{b_{a}(l)}^{\bar{b}_{F}} b d F^{n-k}(b)\right)\right] \\
& =\sum_{k=0}^{n-1} \frac{1}{n-k}\left(\begin{array}{c}
n-1 \\
k
\end{array}\right)\left[q\left(-(1-q)^{k} q^{n-k-1}+\mathcal{I}(k=0)\right)\left(1-\int_{b_{a}(l)}^{\bar{b}_{F}} b d F^{n-k}(b)\right)\right]<0 \\
& =-\sum_{k=1}^{n-1} \frac{1}{n-k}\left(\begin{array}{c}
n-1 \\
k
\end{array}\right)\left[(1-q)^{k} q^{n-k}\left(1-\int_{b_{a}(l)}^{\bar{b}_{F}} b d F^{n-k}(b)\right)\right]+\frac{1}{n} q\left(1-q^{n-1}\right)\left(1-\int_{b_{a}(l)}^{\bar{b}_{F}} b d F^{n}(b)\right)
\end{aligned}
$$

Note that by Lemma $6 \mathrm{~A}$ this derivative is negative.

Note now that $F$ is increasing, $F\left(b_{a}(l)\right)=0$, and $F\left(\bar{b}_{F}\right)=1$. Moreover, $b_{a}(l)<$ $E\left[v \mid n-1 l, h,\left(0, \bar{\alpha}_{1}\right)\right]$ (which is true when ambiguity is not too large) ensures that the 
utility of high type is greater than 0 . Any bid below $b_{a}(l)$ gives zero utility, and any bid above $\bar{b}_{F}$ gives strictly lower utility than bidding $\bar{b}_{F}$. By construction, under beliefs $\left(\alpha_{0}, \alpha_{1}\right)=\left(0, \bar{\alpha}_{1}\right)$, any bid in the support $\left[b_{a}(l), \bar{b}_{F}\right]$ gives the same utility, and any other mixed strategy can give at most this utility.

Lemma 6A There exists an $\bar{n}$ such that for all $n>\bar{n}$,

$$
\sum_{k=1}^{n-1} \frac{1}{n-k}\left(\begin{array}{c}
n-1 \\
k
\end{array}\right)(1-q)^{n-k} q^{k}\left(\int_{b_{a}(l)}^{\bar{b}_{F}} b d F^{n-k}(b)\right)-\frac{1}{n}(1-q)\left(1-(1-q)^{n-1}\right)\left(\int_{b_{a}(l)}^{\bar{b}_{F}} b d F^{n}(b)\right)>0
$$

Proof of Lemma 6A: Below we will focus just on some of the expressions in the sum above that are close to $k=n q$. These expressions will have the term $\int_{b_{a}(l)}^{\bar{b}_{F}} b d F^{n(1-q)}(b)$ in them. We next prove that this term will be bounded away from zero.

Claim: $\lim _{n \rightarrow \infty}\left(\int_{b_{a}(l)}^{\bar{b}_{F}} b d F^{n(1-q)}(b)\right)>0$ and $\lim _{n \rightarrow \infty}\left(\int_{b_{a}(l)}^{\bar{b}_{F}} b d F^{n}(b)\right)>0$.

Proof: Assume that $a$ is very small and consider the equation for the high type to be indifferent between any bid in $\left[b_{a}(l), \bar{b}_{F}\right]$ : for any $b \in\left[b_{a}(l), \bar{b}_{F}\right], F(b)$ solves:

$$
\begin{aligned}
& \sum_{k=0}^{n-1}\left(\begin{array}{c}
n-1 \\
k
\end{array}\right) P\left(k l, n-k-1 h \mid h,\left(0, \bar{\alpha}_{1}\right)\right)\left(E_{\left(0, \bar{\alpha}_{1}\right)}[v \mid k l, n-k h]-b\right) F(b)^{n-k-1} \\
& =P(n-1 l \mid h,(0, a))\left(b-b_{a}(l)\right) \Leftrightarrow \\
& (*) q a(1-b) F(b)^{n-1}-(1-q)^{n} F(b)^{n-1} b+\left(1-\bar{\alpha}_{1}\right) q^{n} F(b)^{n-1}(1-b) \\
& \quad+\sum_{k=1}^{n-1}\left(\begin{array}{c}
n-1 \\
k
\end{array}\right) P(k l, n-k-1 h \mid h,(0, a))\left(E_{\left(0, \bar{\alpha}_{1}\right)}[v \mid k l, n-k h]-b\right) F(b)^{n-k-1} \\
& \quad=P(n-1 l \mid h,(0, a))\left(b-b_{a}(l)\right)
\end{aligned}
$$

Fact: For any $b<\bar{b}_{F}$, either $F(b)^{n} \rightarrow 0$ or $F(b)^{n} \rightarrow \zeta(b)<1$.

Proof: Suppose that for some $b, \bar{b}_{F}>b>b_{a}(l), F(b)^{n} \rightarrow \zeta(b)>0$. Note that as $n$ grows large, by the law of large numbers, the LHS of $(*)$ converges to

$$
q a(1-b) \zeta(b)+q(1-a)(1-b)(\zeta(b))^{q}-(1-q) b(\zeta(b))^{1-q}
$$

while the RHS converges to zero. Therefore, $(*)$ implies

$$
\bar{\alpha}_{1}(\zeta(b))^{q}+\left(1-\bar{\alpha}_{1}\right)(\zeta(b))^{2 q-1}=\frac{1-q}{q} \frac{b}{1-b}
$$

As $\bar{b}_{F}=E[V \mid h]=q$, if $b<\bar{b}_{F}$ the solution will be $\zeta(b)<1$. 
By the claim, for any $b$ either $F(b)^{n} \rightarrow 0$ or $F(b)^{n} \rightarrow \zeta(b)<1$. Note that $\frac{P(v=0 \mid h,(0, a)))}{P(v=1 \mid h,(0, a)))} \frac{b}{1-b}$ is increasing in $b$ and reaches its maximum for $b=\bar{b}_{F}$ where the expression is equal 1. Therefore, we conclude that for any $b<\bar{b}_{F} \lim _{n \rightarrow \infty} F(b)^{n(2 q-1)}<1$ and so $\lim _{n \rightarrow \infty}\left(\int_{b_{a}(l)}^{\bar{b}_{F}} b d F^{n(1-q)}(b)\right)>$ 0 and $\lim _{n \rightarrow \infty}\left(\int_{b_{a}(l)}^{\bar{b}_{F}} b d F^{n}(b)\right)>0$

For now look at the comparison between

$$
\begin{aligned}
& \sum_{k=1}^{n-1} \frac{1}{n-k}\left(\begin{array}{c}
n-1 \\
k
\end{array}\right)(1-q)^{n-k} q^{k}\left(\int_{b_{a}(l)}^{\bar{b}_{F}} b d F^{n-k}(b)\right) \text { and } \\
& \frac{1}{n}(1-q)\left(1-(1-q)^{n-1}\right)\left(\int_{b_{a}(l)}^{\bar{b}_{F}} b d F^{n}(b)\right)
\end{aligned}
$$

as $n$ grows large.

Above we have seen that $\left(\int_{b_{a}(l)}^{\bar{b}_{F}} b d F^{n}(b)\right)$ is bounded from zero, therefore the second term is the same magnitude as $\frac{1}{n}$.

We will consider only part of the first term. Consider the expression for $k=n q$ :

By Stirling's approximation, $\left(\begin{array}{c}n-1 \\ k\end{array}\right)=\frac{(n-1) !}{(n-k-1) ! k !} \simeq \frac{\sqrt{2 \pi n}\left(\frac{n}{e}\right)^{n}}{\sqrt{2 \pi n(1-q)}\left(\frac{n(1-q)}{e}\right)^{n(1-q)} \sqrt{2 \pi n q}\left(\frac{n q}{e}\right)^{n q}}=\sqrt{\frac{1}{n q(1-q)}} \frac{1}{(1-q))^{n(1-q)}}$ so that the term in the sum relating to $k=n q$ becomes: $\frac{1}{n-k}\left(\begin{array}{c}n-1 \\ k\end{array}\right)\left[(1-q)^{n-k} q^{k} \simeq\right.$ $\frac{1}{n(1-q)} \sqrt{\frac{1}{n q(1-q)}}$. For any $n$ take a sequence $n_{1}$ such that $\frac{n_{1}}{n} \rightarrow 0$ and $\frac{n_{1}}{\sqrt{n}} \rightarrow \infty$. Consider all the terms in the sum $k \in\left\{n q-n_{1}, \ldots, n q+n_{1}\right\}$, note that for all these we have that:

$$
\sum_{k \in\left\{n q-n_{1}, \ldots, n q+n_{1}\right\}} \frac{1}{n-k}\left(\begin{array}{c}
n-1 \\
k
\end{array}\right)\left[(1-q)^{n-k} q^{k} \simeq 2 n_{1} \frac{1}{n(1-q)} \sqrt{\frac{1}{n q(1-q)}}\right.
$$

where $\frac{2 n_{1} \frac{1}{n(1-q)} \sqrt{\frac{1}{n q(1-q)}}}{\frac{1}{n}}=\frac{2 n_{1} \frac{1}{(1-q)} \sqrt{\frac{1}{q(1-q)}}}{\sqrt{n}} \rightarrow \infty$. As $\left(\int_{b_{a}(l)}^{\bar{b}_{F}} b d F^{n(1-q)}(b)\right)$ is bounded from zero we are done.

Proposition 6B: In the equilibrium described in Proposition 6A, the seller's revenue is decreasing in a.

Proof. To make clear that the equilibrium strategy depends on $n$ and $a$, denote the bidding strategy for the high type by $F(b ; n, a)$. As in the proof of Lemma $6 \mathrm{~A}$, consider the equation defining $F(b ; n, a)$ :

$$
\begin{aligned}
& q \bar{\alpha}_{1}(1-b) F(b ; n, a)^{n-1}-(1-q)^{n} F(b ; n, a)^{n-1} b+\left(1-\bar{\alpha}_{1}\right) q^{n} F(b ; n, a)^{n-1}(1-b) \\
& +\sum_{k=1}^{n-1}\left(\begin{array}{c}
n-1 \\
k
\end{array}\right) P\left(k l, n-k-1 h \mid h,\left(0, \bar{\alpha}_{1}\right)\right)\left(E_{\left(0, \bar{\alpha}_{1}(a)\right)}[v \mid k l, n-k h]-b\right) F(b ; n, a)^{n-k-1} \\
& =P\left(n-1 l \mid h,\left(0, \bar{\alpha}_{1}\right)\right)\left(b-b_{a}(l)\right)
\end{aligned}
$$

By the law of large numbers, for large $n$, the LHS is close to:

$$
q \bar{\alpha}_{1}(1-b) F(b ; n, a)^{n-1}+q\left(1-\bar{\alpha}_{1}\right)(1-b) F(b ; n, a)^{q(n-1)}-(1-q) b F(b ; n, a)^{(1-q)(n-1)}
$$


and the RHS is close to 0 . Thus, $F(b ; n, a)$ is close to $F^{*}(b ; n, a)$, where for $b \in[0, q]$, $F^{*}(b ; n, a)$ is the unique positive solution to:

$q \bar{\alpha}_{1}(1-b) F(b ; n, a)^{n-1}+q\left(1-\bar{\alpha}_{1}\right)(1-b) F(b ; n, a)^{q(n-1)}-(1-q) b F(b ; n, a)^{(1-q)(n-1)}=0$

Note that $F^{*}(b ; n, a)$ is increasing in $a$. Now when $n$ is large, the expected bid of the high type, $\int_{b_{a}(l)}^{\bar{b}_{F}} b d F(b ; n, a)$ is close to $\int_{0}^{q} b d F^{*}(b ; n, a)$. Take $a^{\prime}>a$; since $F^{*}(b ; n, a)$ first order stochastically dominates $F^{*}\left(b ; n, a^{\prime}\right), \int_{0}^{q} b d F^{*}\left(b ; n, a^{\prime}\right)<\int_{0}^{q} b d F^{*}(b ; n, a)$, which for large $n$ implies $\int_{b_{a}(l)}^{\bar{b}_{F}} b d F\left(b ; n, a^{\prime}\right)<\int_{b_{a}(l)}^{\bar{b}_{F}} b d F(b ; n, a)$. Thus, the expected payment of the high type is decreasing in $a$. Since the expected payment of the low type is $E_{\left(\bar{\alpha}_{0}(a), 0\right)}[v \mid l, \ldots, l]$ which is decreasing in $a$, this implies that the seller's revenue is decreasing in $a$.

\subsubsection{Continuous signals}

Here we consider a general set of marginal distributions, $g_{v}(s)$. Specifically assume that $g_{1}$ is increasing and $g_{0}$ is decreasing, and assume symmetry so that $g_{1}(s)=g_{0}(1-s)$.

Let us first write the utility of a player per each bid $b$. This is

$$
U\left(s^{1}, b\right) \propto \min _{\boldsymbol{\lambda}}\left(\int_{0}^{z}\left(1-b\left(s^{\prime}\right)\right) f_{1}\left(s^{1}, s^{\prime}\right) d s^{\prime}-\int_{0}^{z} b\left(s^{\prime}\right) f_{0}\left(s^{1}, s^{\prime}\right) d s^{\prime}\right)
$$

where $b\left(s^{\prime}\right)$ is the bid used by the other player and $z=b^{-1}(b)$. Thus per each bid $b$, each player minimizes his utility by choosing a vector $\boldsymbol{\lambda}$, given the strategy of the other player. Recall that $s_{v}$, for $v \in\{0,1\}$, is the median of the cdf $G_{v}()$.

Lemma A1: Consider an equilibrium in which $b(s)$ is increasing. Let $\boldsymbol{\lambda}_{v}^{*}(s)$ denote the information structure which minimizes the utility of the player for each s. Then:

(i) $\left(\lambda_{0}^{*}, \lambda_{1}^{*}\right)=\left(\lambda_{\max }, \lambda_{\min }\right)$ for all $s<s_{0}$.

(ii) $\left(\lambda_{0}^{*}, \lambda_{1}^{*}\right)=\left(\lambda_{\min }, \lambda_{\min }\right)$ for all $s \in\left[s_{0}, \min \left\{\hat{s}, s_{1}\right\}\right]$.

(iii) $\left(\lambda_{0}^{*}, \lambda_{1}^{*}\right)=\left(\lambda_{\min }, \lambda_{\max }\right)$ in $\left[s_{1}, \hat{s}\right]$ if $s_{1}<\hat{s}$ and $\left(\lambda_{0}, \lambda_{1}\right)=\left(\lambda_{\max }, \lambda_{\min }\right)$ in $\left[\hat{s}, s_{1}\right]$ otherwise.

(iv) $\left(\lambda_{0}^{*}, \lambda_{1}^{*}\right)=\left(\lambda_{\max }, \lambda_{\max }\right)$ for all $s>\max \left\{s_{1}, \hat{s}\right\}$,

and $\hat{s}<1$ satisfies

$$
\left.\int_{0}^{\hat{s}} b\left(s^{\prime}\right) g_{0}\left(s^{\prime}\right)\left(2 G_{0}\left(s^{\prime}\right)-1\right)\right) d s^{\prime}=0
$$

That is, $\boldsymbol{\lambda}^{*}(s)$ changes with $s$, so the behaviour as described cannot be rationalized with a unique a priori $\boldsymbol{\lambda}$.

Proofs of Lemma A1 and Proposition 7: We first show in Claims 1-3 how players choose $\boldsymbol{\lambda}^{*}$ to minimize their utility given each $s$, when the bid of the other player is 
weakly increasing in $s^{\prime}$. We then show that the bidding function described above, for the $\boldsymbol{\lambda}$ s chosen, is an equilibrium.

Define:

$$
\begin{aligned}
& I_{1}(s)=\int_{0}^{s}\left(1-b\left(s^{\prime}\right)\right) g_{1}\left(s^{\prime}\right) g_{1}(s)\left(2 G_{1}(s)-1\right)\left(2 G_{1}\left(s^{\prime}\right)-1\right) d s^{\prime} \\
& \left.I_{0}(s)=-\int_{0}^{s} b\left(s^{\prime}\right) g_{0}\left(s^{\prime}\right) g_{0}(s)\left(2 G_{0}(s)-1\right)\left(2 G_{0}\left(s^{\prime}\right)-1\right)\right) d s^{\prime}
\end{aligned}
$$

Thus:

Claim 1: In equilibrium, $\lambda_{v}^{*}=\lambda_{\min }\left(\lambda_{\max }\right)$ iff $I_{v}(s)>(<) 0$.

$I_{v}(s)$ is the derivative of the expected utility with respect to $\lambda_{v}$. Given max-min behaviour, the statement follows.

Claim 2: (i) $I_{1}(s)>0$ for $s<s_{1}, I_{1}(s)<0$ for all $s>s_{1}$; (ii) $I_{0}(s)<0$ for $s<s_{0}$, $I_{0}(s)>0$ for all $s \in\left(s_{0}, \hat{s}\right), I_{0}(s)<0$ for all $s>\hat{s}$.

\section{Proof of Claim 2:}

(i) $I_{1}(s)$ : This function must be strictly positive for $s<s_{1}$ as $\left(2 G_{1}(s)-1\right)\left(2 G_{1}\left(s^{\prime}\right)-1\right)>$ 0 for $s, s^{\prime}<s_{1}$. Note that $I_{1}\left(s_{1}\right)=0$, and that

$$
\begin{aligned}
\left.\frac{\partial I_{1}(s)}{\partial s}\right|_{s=s_{1}} & =\left.\frac{\partial g_{1}(s)\left(2 G_{1}(s)-1\right)}{\partial s}\right|_{s=s_{1}} \int_{0}^{s_{1}}\left(1-b\left(s^{\prime}\right)\right) g_{1}\left(s^{\prime}\right)\left(2 G_{1}\left(s^{\prime}\right)-1\right) d s^{\prime} \\
& =2\left(g_{1}\left(s_{1}\right)\right)^{2} \int_{0}^{s_{1}}\left(1-b\left(s^{\prime}\right)\right) g_{1}\left(s^{\prime}\right)\left(2 G_{1}\left(s^{\prime}\right)-1\right) d s^{\prime}<0
\end{aligned}
$$

More generally:

$$
\begin{aligned}
\frac{\partial I_{1}(s)}{\partial s}= & \left(g_{1}^{\prime}(s)\left(2 G_{1}(s)-1\right)+2\left(g_{1}(s)\right)^{2}\right) \int_{0}^{s}\left(1-b\left(s^{\prime}\right)\right) g_{1}\left(s^{\prime}\right)\left(2 G_{1}\left(s^{\prime}\right)-1\right) d s^{\prime} \\
& +(1-b(s)) g_{1}(s) g_{1}(s)\left(2 G_{1}(s)-1\right)\left(2 G_{1}(s)-1\right) \\
= & \left.\left.\left(\frac{g_{1}^{\prime}(s)}{g_{1}(s)}+\frac{2\left(g_{1}(s)\right)}{\left(2 G_{1}(s)-1\right)}\right) I_{1}(s)+2\left(g_{1}(s)\right)^{2}\right)(1-b(s))\left(2 G_{1}(s)-1\right)\left(2 G_{1}(s)-1\right)\right)
\end{aligned}
$$

So whenever $I_{1}(s)>0$ and $s>s_{1}$ we have that $\frac{\partial I_{1}(s)}{\partial s}>0$ as $g_{1}(s)$ is increasing and $\left.\left.2\left(g_{1}(s)\right)^{2}\right)(1-b(s))\left(2 G_{1}(s)-1\right)\left(2 G_{1}(s)-1\right)\right)>0$. So now it suffices to check that $I_{1}(1)<0$ :

$$
\begin{aligned}
I_{1}(1) & =g_{1}(1) \int_{0}^{1}\left(1-b\left(s^{\prime}\right)\right) g_{1}\left(s^{\prime}\right)\left(2 G_{1}\left(s^{\prime}\right)-1\right) d s^{\prime} \\
& =g_{1}(1) \int_{0}^{s_{1}}\left(1-b\left(s^{\prime}\right)\right) g_{1}\left(s^{\prime}\right)\left(2 G_{1}\left(s^{\prime}\right)-1\right) d s^{\prime}+g_{1}(1) \int_{s_{1}}^{1}(1-b(s)) g_{1}\left(s^{\prime}\right)\left(2 G_{1}\left(s^{\prime}\right)-1\right) d s^{\prime} \\
& <g_{1}(1) \int_{0}^{s_{1}}\left(1-b\left(s_{1}\right)\right) g_{1}\left(s^{\prime}\right)\left(2 G_{1}\left(s^{\prime}\right)-1\right) d s^{\prime}+g_{1}(1) \int_{s_{1}}^{1}\left(1-b\left(s_{1}\right)\right) g_{1}\left(s^{\prime}\right)\left(2 G_{1}\left(s^{\prime}\right)-1\right) d s^{\prime} \\
& =g_{1}(1) \int_{0}^{s_{1}}\left(1-b\left(s_{1}\right)\right) g_{1}\left(s^{\prime}\right)\left(2 G_{1}\left(s^{\prime}\right)-1\right) d s^{\prime}=0
\end{aligned}
$$


where the last inequality follows as $b\left(s^{\prime}\right)$ is increasing, $\left(2 G_{1}\left(s^{\prime}\right)-1\right)>0(<0)$ whenever $s>s_{1}\left(s<s_{1}\right)$. The last equality follows from $\int_{0}^{1} g_{1}\left(s^{\prime}\right)\left(2 G_{1}\left(s^{\prime}\right)-1\right) d s^{\prime}=0$.

(ii) $I_{0}(s)$ : This function must be strictly negative for $s<s_{0}$ as $\left(2 G_{0}(s)-1\right)\left(2 G_{0}\left(s^{\prime}\right)-\right.$ 1) $>0$ for $s, s^{\prime}<s_{0}$. Note that $I_{0}\left(s_{0}\right)=0$. Moreover,

$$
\begin{aligned}
\left.\frac{\partial I_{0}(s)}{\partial s}\right|_{s=s_{0}}= & -\left.\frac{\partial g_{0}(s)\left(2 G_{0}(s)-1\right)}{\partial s}\right|_{s=s_{0}} \int_{0}^{s_{0}} b\left(s^{\prime}\right) g_{0}\left(s^{\prime}\right)\left(2 G_{0}\left(s^{\prime}\right)-1\right) d s^{\prime} \\
& \left.-b\left(s^{\prime}\right) g_{0}\left(s^{\prime}\right) g_{0}\left(s_{0}\right)\left(2 G_{0}\left(s_{0}\right)-1\right)\left(2 G_{0}\left(s^{\prime}\right)-1\right)\right) \\
= & -2\left(g_{0}\left(s_{0}\right)\right)^{2} \int_{0}^{s_{0}} b\left(s^{\prime}\right) g_{0}\left(s^{\prime}\right)\left(2 G_{0}\left(s^{\prime}\right)-1\right) d s^{\prime}>0
\end{aligned}
$$

So $I_{0}(s)<0$ for $s \gtrsim s_{0}$. Note that $\left.-\int_{0}^{s} b\left(s^{\prime}\right) g_{0}\left(s^{\prime}\right)\left(2 G_{0}\left(s^{\prime}\right)-1\right)\right) d s^{\prime}$ is decreasing for $s>s_{0}$. Thus if $I_{0}(1)<0$, we have the result. But

$$
\begin{aligned}
\left|I_{0}(1)\right|= & g_{0}(1) \int_{0}^{1} b\left(s^{\prime}\right) g_{0}\left(s^{\prime}\right)\left(2 G_{0}\left(s^{\prime}\right)-1\right) d s^{\prime} \\
> & g_{0}(1) \int_{0}^{s_{0}} b\left(s_{0}\right) g_{0}\left(s^{\prime}\right)\left(2 G_{0}\left(s^{\prime}\right)-1\right) d s^{\prime} \\
& +g_{0}(1) \int_{s_{0}}^{1} b\left(s_{0}\right) g_{0}\left(s^{\prime}\right)\left(2 G_{0}\left(s^{\prime}\right)-1\right) d s^{\prime} \\
= & g_{0}(1) b\left(s_{0}\right) \int_{0}^{1} g_{0}\left(s^{\prime}\right)\left(2 G_{0}\left(s^{\prime}\right)-1\right) d s^{\prime}=0 .
\end{aligned}
$$

Thus we know there exists $\hat{s}<1$ such that:

$$
\left.\int_{0}^{\hat{s}} b\left(s^{\prime}\right) g_{0}\left(s^{\prime}\right)\left(2 G_{0}\left(s^{\prime}\right)-1\right)\right) d s^{\prime}=0
$$

and we can conclude that $I_{0}(s)>0$ for $s \in\left(s_{0}, \hat{s}\right)$ and that $I_{0}(s)<0$ for $s>\hat{s}$.

Consider now the bidding function $E^{\lambda^{*}}(v \mid s, s)$. Note that overbidding, compared to the canonical model, arises when

$$
\frac{\left[1+\lambda_{1}\left(2 G_{1}(s)-1\right)^{2}\right] g_{1}^{2}(s)}{\left[1+\lambda_{1}\left(2 G_{1}(s)-1\right)^{2}\right] g_{1}^{2}(s)+\left[1+\lambda_{1}\left(2 G_{0}(s)-1\right)^{2}\right] g_{0}^{2}(s)}>\frac{g_{1}^{2}(s)}{g_{1}^{2}(s)+g_{0}^{2}(s)}
$$

which holds if an only if:

$$
\frac{\left[1+\lambda_{1}\left(2 G_{1}\left(s^{\prime}\right)-1\right)^{2}\right]}{\left[1+\lambda_{0}\left(2 G_{0}\left(s^{\prime}\right)-1\right)^{2}\right]}>1
$$

We then have:

Claim 3: When $b(s)=E^{\lambda^{*}}(v \mid s, s)$, a necessary condition for overbidding compared to the canonical model is $\hat{s}>0.5$, that is:

$$
\int_{0}^{0.5} b\left(s^{\prime}\right) g_{0}\left(s^{\prime}\right)\left(2 G_{0}\left(s^{\prime}\right)-1\right) d s^{\prime}<0
$$


If this holds, there is overbidding in the region $\left[s_{0}, \hat{s}\right]$, and underbidding for any other $s$. Otherwise, all types underbid compared to the canonical model.

Proof of Claim 3: Given Claims 1 and 2, we can then deduce the different values of $\lambda_{v}^{*}$ in equilibrium and consider when overbidding/underbidding arises compared to the canonical model when the bidding function is as described in the Proposition.

(i) $\left(\lambda_{0}, \lambda_{1}\right)=\left(\lambda_{\max }, \lambda_{\min }\right)$ for all $s<s_{0}$. As a result, if this is an equilibrium, we would have underbidding as

$$
\frac{\left[1+\lambda_{\min }\left(2 G_{1}\left(s^{\prime}\right)-1\right)^{2}\right]}{\left[1+\lambda_{\max }\left(2 G_{0}\left(s^{\prime}\right)-1\right)^{2}\right]}<1,
$$

which is indeed the case as $\lambda_{\min }<0<\lambda_{\max }$.

b. $\left(\lambda_{0}, \lambda_{1}\right)=\left(\lambda_{\min }, \lambda_{\min }\right)$ for all $s \in\left[s_{0}, \min \left\{\hat{s}, s_{1}\right\}\right]$. We have underbidding iff:

$$
\frac{\left[1+\lambda_{\min }\left(2 G_{1}\left(s^{\prime}\right)-1\right)^{2}\right]}{\left[1+\lambda_{\min }\left(2 G_{0}\left(s^{\prime}\right)-1\right)^{2}\right]}<1
$$

If $\min \left\{\hat{s}, s_{1}\right\}>0.5$, then we would have overbidding because in the region above 0.5 , as $\left(2 G_{1}(0.5)-1\right)^{2}=\left(2 G_{0}(0.5)-1\right)^{2}$ by symmetry, but because of convexity (concavity) of $G_{1}$ $\left(G_{0}\right)$, the fraction would be greater than 1 , as we would have $\left(2 G_{1}\left(s^{\prime}\right)-1\right)^{2}<\left(2 G_{0}\left(s^{\prime}\right)-1\right)^{2}$ just above 0.5 .

c. $\left(\lambda_{0}, \lambda_{1}\right)=\left(\lambda_{\max }, \lambda_{\max }\right)$ for all $s>\max \left\{s_{1}, \hat{s}\right\}$. In this case we also have underbidding as $\left[1+\lambda_{\max }\left(2 G_{1}\left(s^{\prime}\right)-1\right)^{2}\right]<\left[1+\lambda_{\max }\left(2 G_{0}\left(s^{\prime}\right)-1\right)^{2}\right]$, because $\frac{1}{2}<G_{1}\left(s^{\prime}\right)<G_{0}\left(s^{\prime}\right)$.

d. If $0.5<s_{1}<\hat{s}$ : in the region $\left[s_{1}, \hat{s}\right]$ we have $\left(\lambda_{0}, \lambda_{1}\right)=\left(\lambda_{\min }, \lambda_{\max }\right)$. In this case we have overbidding as:

$$
\frac{\left[1+\lambda_{\max }\left(2 G_{1}\left(s^{\prime}\right)-1\right)^{2}\right]}{\left[1+\lambda_{\min }\left(2 G_{0}\left(s^{\prime}\right)-1\right)^{2}\right]}>1
$$

For this we need $s_{1}<\hat{s}$, implying that $0.5<\hat{s}$.

e. if $\hat{s}<s_{1}$ : Then we have $\left(\lambda_{0}, \lambda_{1}\right)=\left(\lambda_{\max }, \lambda_{\min }\right)$ in this region between the two values. Then we have underbidding as:

$$
\frac{\left[1+\lambda_{\min }\left(2 G_{1}\left(s^{\prime}\right)-1\right)^{2}\right]}{\left[1+\lambda_{\max }\left(2 G_{0}\left(s^{\prime}\right)-1\right)^{2}\right]}<1 .
$$

Thus the structure of the equilibrium is therefore as above. So for overbidding we need:

$$
\begin{aligned}
& \int_{0}^{0.5} b\left(s^{\prime}\right) g_{0}\left(s^{\prime}\right)\left(2 G_{0}\left(s^{\prime}\right)-1\right) d s^{\prime} \\
= & \int_{0}^{s_{0}} \frac{\left[1+\lambda_{\min }\left(2 G_{1}\left(s^{\prime}\right)-1\right)^{2}\right] g_{1}^{2}\left(s^{\prime}\right)}{\left[1+\lambda_{\min }\left(2 G_{1}\left(s^{\prime}\right)-1\right)^{2}\right] g_{1}^{2}\left(s^{\prime}\right)+\left[1+\lambda_{\max }\left(2 G_{0}\left(s^{\prime}\right)-1\right)^{2}\right] g_{0}^{2}\left(s^{\prime}\right)} g_{0}\left(s^{\prime}\right)\left(2 G_{0}\left(s^{\prime}\right)-1\right) d s^{\prime} \\
& +\int_{s_{0}}^{0.5} \frac{\left[1+\lambda_{\min }\left(2 G_{1}\left(s^{\prime}\right)-1\right)^{2}\right] g_{1}^{2}\left(s^{\prime}\right)}{\left[1+\lambda_{\min }\left(2 G_{1}\left(s^{\prime}\right)-1\right)^{2}\right] g_{1}^{2}\left(s^{\prime}\right)+\left[1+\lambda_{\min }\left(2 G_{0}\left(s^{\prime}\right)-1\right)^{2}\right] g_{0}^{2}\left(s^{\prime}\right)} g_{0}\left(s^{\prime}\right)\left(2 G_{0}\left(s^{\prime}\right)-1\right) d s^{\prime} \\
< & 0
\end{aligned}
$$


which is analogous to what is in the Proposition. Finally we need to show that the construction above is an equilibrium:

Claim 4: The bidding function $b\left(s^{\prime}\right)$ defined above with the values of $\boldsymbol{\lambda}^{*}(s)$ described above consists a symmetric equilibrium when $a$ is low enough.

Proof of Claim 4: We now show that given the above it is optimal, wlog, for player 1 to choose $b(s)$ at $s$, when player 2 uses $b\left(s^{\prime}\right)$ and $\boldsymbol{\lambda}^{*}(s)$ as defined above.

Let $\hat{\boldsymbol{\lambda}}$ equal $\boldsymbol{\lambda}^{*}(s)$ and consider the virtual utility:

$$
\begin{aligned}
\hat{U}(s, z) & =\int_{0}^{z}\left(E^{\hat{\boldsymbol{\lambda}}(s)}\left(v \mid s, s^{\prime}\right)-b\left(s^{\prime}\right)\right) d F^{\hat{\boldsymbol{\lambda}}(s)}\left(s, s^{\prime}\right) \\
& =\frac{1}{2}\left(\int_{0}^{z}\left(\left(1-b\left(s^{\prime}\right)\right) f_{1}\left(\hat{\boldsymbol{\lambda}}, s, s^{\prime}\right)-b\left(s^{\prime}\right) f_{0}\left(\hat{\boldsymbol{\lambda}}, s, s^{\prime}\right)\right) d s^{\prime}\right.
\end{aligned}
$$

This is not player 1's utility as it is evaluated at $\hat{\boldsymbol{\lambda}}$ for all $s^{\prime}$. However note that when $z=s$, then the integrand is zero. To see that the integrand equals 0 note that, as $\hat{\boldsymbol{\lambda}}=\boldsymbol{\lambda}^{*}(s)$,

$$
(1-b(s)) f_{1}(\hat{\boldsymbol{\lambda}}, s, s)=b(s) f_{0}(\hat{\boldsymbol{\lambda}}, s, s)
$$

iff

$$
\begin{aligned}
& {\left[1+\lambda_{0}^{*}\left(2 G_{0}(s)-1\right)^{2}\right] g_{0}^{2}(s)\left[1+\lambda_{1}^{*}\left(2 G_{1}(s)-1\right)\left(2 G_{1}(s)-1\right)\right] g_{1}(s) g_{1}(s) } \\
= & {\left[1+\lambda_{1}^{*}\left(2 G_{1}(s)-1\right)^{2}\right] g_{1}^{2}(s)\left[1+\lambda_{0}^{*}\left(2 G_{0}(s)-1\right)\left(2 G_{0}(s)-1\right)\right] g_{0}(s) g_{0}(s) }
\end{aligned}
$$

which holds.

Moreover as we now show the first order condition w.r.t. $s^{\prime}$ is zero, the second order condition evaluated at this point is negative, thus $z=s$ is a maximum. To see this, suppose that we have a $z$ for which $\hat{U}(s, z)=0$. Taking a second derivative w.r.t. $z$ we get: $-b^{\prime}(z)\left(f_{1}(\hat{\boldsymbol{\lambda}}, s, z)+f_{0}(\hat{\boldsymbol{\lambda}}, s, z)\right)+(1-b(z)) f_{1}^{\prime}(\hat{\boldsymbol{\lambda}}, s, z)-b(z) f_{0}^{\prime}(\hat{\boldsymbol{\lambda}}, s, z)$. As $\hat{U}(s, z)=0$, this implies that $(1-b(z))=\frac{b(z) f_{0}(\hat{\boldsymbol{\lambda}}, s, z)}{f_{1}(\hat{\boldsymbol{\lambda}}, s, z)}$, and thus the second order derivative at that $z$ is

$$
\begin{aligned}
& -b^{\prime}(z)\left(f_{1}(\hat{\boldsymbol{\lambda}}, s, z)+f_{0}(\hat{\boldsymbol{\lambda}}, s, z)\right)+\frac{b(z) f_{0}(\hat{\boldsymbol{\lambda}}, s, z)}{f_{1}(\hat{\boldsymbol{\lambda}}, s, z)} f_{1}^{\prime}(\hat{\boldsymbol{\lambda}}, s, z)-b(z) f_{0}^{\prime}(\hat{\boldsymbol{\lambda}}, s, z) \\
= & -b^{\prime}(z)\left(f_{1}(\hat{\boldsymbol{\lambda}}, s, z)+f_{0}(\hat{\boldsymbol{\lambda}}, s, z)\right)+b(z)\left(\frac{f_{0}(\hat{\boldsymbol{\lambda}}, s, z)}{f_{1}(\hat{\boldsymbol{\lambda}}, s, z)} f_{1}^{\prime}(\hat{\boldsymbol{\lambda}}, s, z)-f_{0}^{\prime}(\hat{\boldsymbol{\lambda}}, s, z)\right)
\end{aligned}
$$

Note that the first element is always negative. The second element is negative iff: $\frac{\left.g_{1}^{\prime}(z) g_{1}(s)\left(1+\hat{\boldsymbol{\lambda}}_{1}\left(2 G_{1}(z)-1\right)\left(2 G_{1}(s)-1\right)\right)+g_{1}(z) g_{1}(s) \hat{\boldsymbol{\lambda}}_{1} 2 g_{1}(z)\left(2 G_{1}(s)-1\right)\right)}{\left.g_{0}^{\prime}(z) g_{0}(s)\left(1+\hat{\boldsymbol{\lambda}}_{0}\left(2 G_{0}(z)-1\right)\left(2 G_{0}(s)-1\right)\right)+g_{0}(z) g_{0}(s) \hat{\boldsymbol{\lambda}}_{0} 2 g_{0}(z)\left(2 G_{0}(s)-1\right)\right)}<\frac{g_{1}(z) g_{1}(s)\left(1+\hat{\boldsymbol{\lambda}}_{1}\left(2 G_{1}(z)-1\right)\left(2 G_{1}(s)-1\right)\right)}{g_{0}(z) g_{0}(s)\left(1+\hat{\boldsymbol{\lambda}}_{0}\left(2 G_{0}(z)-1\right)\left(2 G_{0}(s)-1\right)\right)}$ Note that when $\hat{\boldsymbol{\lambda}}$ is small enough, this is always the case as the LHS is negative. Thus a solution to the first order condition is unique. 
But the above implies that player 1 can achieve this utility above and cannot improve upon it when using other bids $z \neq s$.

So we know that the player bids until the integrand gets negative, so, written differently, until $E^{\hat{\boldsymbol{\lambda}}(s)}(v \mid s, s)=b(s)$, which gives us the equilibrium bidding function.

We now consider the seller's revenue and show they decrease in $a$, under the sufficient condition identified. Consider the case when $\hat{s}>s_{1}$. Let

$$
w\left(s^{\prime}\right)=\left(1-G_{1}\left(s^{\prime}\right)\right) g_{1}\left(s^{\prime}\right)+\left(1-G_{0}\left(s^{\prime}\right)\right) g_{0}\left(s^{\prime}\right)
$$

The seller's revenue can be written as:

$$
\begin{aligned}
R(a)= & \int_{0}^{s_{0}} b\left(s^{\prime}, \lambda_{\max }, \lambda_{\min }\right) w\left(s^{\prime}\right) d s^{\prime}+\int_{s_{0}}^{s_{1}} b\left(s^{\prime}, \lambda_{\min }, \lambda_{\min }\right) w\left(s^{\prime}\right) d s^{\prime}+ \\
& \int_{s_{1}}^{\hat{s}} b\left(s^{\prime}, \lambda_{\min }, \lambda_{\max }\right) w\left(s^{\prime}\right) d s^{\prime}+\int_{\hat{s}}^{1} b\left(s^{\prime}, \lambda_{\max }, \lambda_{\max }\right) w\left(s^{\prime}\right) d s^{\prime}
\end{aligned}
$$

The derivative w.r.t. $a$ is:

$$
\begin{aligned}
\frac{\partial R(a)}{\partial a}= & \int_{0}^{s_{0}} \frac{\partial}{\partial a} b\left(s^{\prime}, \lambda_{\max }, \lambda_{\min }\right) w\left(s^{\prime}\right) d s^{\prime}+\int_{s_{0}}^{s_{1}} \frac{\partial}{\partial a} b\left(s^{\prime}, \lambda_{\min }, \lambda_{\min }\right) w\left(s^{\prime}\right) d s^{\prime}+ \\
& \int_{s_{1}}^{\hat{s}} \frac{\partial}{\partial a} b\left(s^{\prime}, \lambda_{\min }, \lambda_{\max }\right) w\left(s^{\prime}\right) d s^{\prime}+\int_{\hat{s}}^{1} \frac{\partial}{\partial a} b\left(s^{\prime}, \lambda_{\max }, \lambda_{\max }\right) w\left(s^{\prime}\right) d s^{\prime}+ \\
& \frac{\partial \hat{s}}{\partial a}\left(b\left(\hat{s}, \lambda_{\min }, \lambda_{\max }\right)-b\left(\hat{s}, \lambda_{\max }, \lambda_{\max }\right)\right) w(\hat{s})
\end{aligned}
$$

We note that

$$
\begin{aligned}
\left.\frac{\partial}{\partial a} b\left(s^{\prime}, \lambda_{\max }, \lambda_{\min }\right)\right|_{a=1} & =\frac{g_{1}\left(s^{\prime}\right)^{2} g_{0}\left(s^{\prime}\right)^{2}\left[-\left(2 G_{1}\left(s^{\prime}\right)-1\right)^{2}-\left(2 G_{0}\left(s^{\prime}\right)-1\right)^{2}\right]}{\left(g_{1}\left(s^{\prime}\right)^{2}+g_{0}\left(s^{\prime}\right)^{2}\right)^{2}} \\
\left.\frac{\partial}{\partial a} b\left(s^{\prime}, \lambda_{\min }, \lambda_{\min }\right)\right|_{a=1} & =\frac{g_{1}\left(s^{\prime}\right)^{2} g_{0}\left(s^{\prime}\right)^{2}\left[-\left(2 G_{1}\left(s^{\prime}\right)-1\right)^{2}+\left(2 G_{0}\left(s^{\prime}\right)-1\right)^{2}\right]}{\left(g_{1}\left(s^{\prime}\right)^{2}+g_{0}\left(s^{\prime}\right)^{2}\right)^{2}} \\
\left.\frac{\partial}{\partial a} b\left(s^{\prime}, \lambda_{\min }, \lambda_{\max }\right)\right|_{a=1} & =\frac{g_{1}\left(s^{\prime}\right)^{2} g_{0}\left(s^{\prime}\right)^{2}\left[\left(2 G_{1}\left(s^{\prime}\right)-1\right)^{2}+\left(2 G_{0}\left(s^{\prime}\right)-1\right)^{2}\right]}{\left(g_{1}\left(s^{\prime}\right)^{2}+g_{0}\left(s^{\prime}\right)^{2}\right)^{2}} \\
\left.\frac{\partial}{\partial a} b\left(s^{\prime}, \lambda_{\max }, \lambda_{\max }\right)\right|_{a=1} & =\frac{g_{1}\left(s^{\prime}\right)^{2} g_{0}\left(s^{\prime}\right)^{2}\left[\left(2 G_{1}\left(s^{\prime}\right)-1\right)^{2}-\left(2 G_{0}\left(s^{\prime}\right)-1\right)^{2}\right]}{\left(g_{1}\left(s^{\prime}\right)^{2}+g_{0}\left(s^{\prime}\right)^{2}\right)^{2}}
\end{aligned}
$$

So that:

$$
\begin{aligned}
\left.\frac{\partial}{\partial a} b\left(s^{\prime}, \lambda_{\max }, \lambda_{\min }\right)\right|_{a=1} & =-\left.\frac{\partial}{\partial a} b\left(s^{\prime}, \lambda_{\min }, \lambda_{\max }\right)\right|_{a=1}=-\left.\frac{\partial}{\partial a} b\left(1-s^{\prime}, \lambda_{\min }, \lambda_{\max }\right)\right|_{a=1} \\
\left.\frac{\partial}{\partial a} b\left(s^{\prime}, \lambda_{\max }, \lambda_{\max }\right)\right|_{a=1} & =-\left.\frac{\partial}{\partial a} b\left(s^{\prime}, \lambda_{\min }, \lambda_{\min }\right)\right|_{a=1}=\left.\frac{\partial}{\partial a} b\left(1-s^{\prime}, \lambda_{\min }, \lambda_{\min }\right)\right|_{a=1}
\end{aligned}
$$


And therefore we can write $\left.\frac{\partial R(a)}{\partial a}\right|_{a=1}$ as:

$$
\begin{aligned}
\left.\frac{\partial R(a)}{\partial a}\right|_{a=1}= & -\int_{0}^{1-\hat{s}} \frac{\partial}{\partial a} b\left(s^{\prime}, \lambda_{\min }, \lambda_{\max }\right) w\left(s^{\prime}\right) d s^{\prime}-\int_{1-\hat{s}}^{s_{0}} \frac{\partial}{\partial a} b\left(s^{\prime}, \lambda_{\min }, \lambda_{\max }\right)\left[w\left(s^{\prime}\right)-w\left(1-s^{\prime}\right)\right] d s^{\prime} \\
& -\int_{s_{0}}^{0.5} \frac{\partial}{\partial a} b\left(s^{\prime}, \lambda_{\max }, \lambda_{\max }\right)\left[w\left(s^{\prime}\right)-w\left(1-s^{\prime}\right)\right] d s^{\prime}-\int_{\hat{s}}^{1} \frac{\partial}{\partial a} b\left(s^{\prime}, \lambda_{\min }, \lambda_{\min }\right) w\left(s^{\prime}\right) d s^{\prime} .
\end{aligned}
$$

Thus, a sufficient condition for revenue to be decreasing in $a$ is $w\left(s^{\prime}\right)$ decreasing over $[\hat{s}, 1-\hat{s}]$

Private values: Consider the following model where each buyer has private valuation $v_{i} \in[0,1]$, independently distributed according to a uniform distribution. Suppose that the buyers believe that the joint distribution is given by the F-G-M copula $f\left(v_{i}, v_{j} ; \lambda\right)=$ $1+\lambda\left(2 v_{i}-1\right)\left(2 v_{j}-1\right)$, where $\lambda \in \Lambda=[\underline{\lambda}, \bar{\lambda}]$. Assume that $0 \in \Lambda$, and define:

$$
G_{\lambda}\left(z \mid v_{i}\right)=\int_{0}^{z}\left(1+\lambda\left(2 v_{i}-1\right)\left(2 v_{j}-1\right)\right) d v_{j}=z+\lambda z(z-1)\left(2 v_{i}-1\right)
$$

That is, $G_{\lambda}\left(\cdot \mid v_{i}\right)$ is the distribution of $v_{j}$ conditional on $v_{i}$, when the belief about the joint distribution is given by $\lambda$. Let $g_{\lambda}\left(\cdot \mid v_{i}\right)$ denote the corresponding density. Define:

$$
L_{\lambda}\left(y \mid v_{i}\right)=\exp \left(-\int_{y}^{v_{i}} \frac{g_{\lambda}(t \mid t)}{G_{\lambda}(t \mid t)} d t\right)
$$

Proposition 8A: When ambiguity is not too large, a symmetric equilibrium in the first price auction is:

$$
b\left(v_{i}\right)= \begin{cases}\int_{0}^{v_{i}} y d L_{\underline{\lambda}}\left(y \mid v_{i}\right) & v_{i} \leq 0.5 \\ \int_{0}^{v_{i}} y d L_{\bar{\lambda}}\left(y \mid v_{i}\right)+\int_{0}^{0.5} y d\left(L_{\underline{\lambda}}(y \mid 0.5)-L_{\bar{\lambda}}(y \mid 0.5)\right) & v_{i}>0.5\end{cases}
$$

Bids in the first price auction are uniformly higher than the case without ambiguity.

Proof of Proposition 8A: Assume that player $j$ is following the equilibrium strategy. Player $i$ 's utility from bidding $b(z) \in\left[0, v_{i}\right]$ is:

$$
\min _{\lambda \in \Lambda} U_{\lambda}\left(z \mid v_{i}\right) \equiv G_{\lambda}\left(z \mid v_{i}\right)\left(v_{i}-b(z)\right)
$$

Note that $G_{\lambda}\left(z \mid v_{i}\right)=z+\lambda z(z-1)\left(2 v_{i}-1\right)$. Thus, $\underline{\lambda}$ minimizes this utility for types $v_{i} \leq 0.5$, and $\bar{\lambda}$ minimizes this utility for types $v_{i}>0.5$. Thus, the utility from bidding $b(z)$ is $U_{\underline{\lambda}}\left(z \mid v_{i}\right)$ for $v_{i} \leq 0.5$ and $U_{\bar{\lambda}}\left(z \mid v_{i}\right)$ for $v_{i}>0.5$. First order conditions imply:

$$
\begin{array}{ll}
g_{\underline{\lambda}}\left(v_{i} \mid v_{i}\right)\left(v_{i}-b\left(v_{i}\right)\right)-G_{\underline{\lambda}}\left(v_{i} \mid v_{i}\right) b^{\prime}\left(v_{i}\right)=0 & v_{i} \leq 0.5 \\
g_{\bar{\lambda}}\left(v_{i} \mid v_{i}\right)\left(v_{i}-b\left(v_{i}\right)\right)-G_{\bar{\lambda}}\left(v_{i} \mid v_{i}\right) b^{\prime}\left(v_{i}\right)=0 & v_{i}>0.5
\end{array}
$$


Using boundary conditions $b(0)=0$ and $b(0.5)=\int_{0}^{0.5} y d L_{\underline{\lambda}}(y \mid 0.5)$, the solution to these differential equations is:

$$
b\left(v_{i}\right)= \begin{cases}\int_{0}^{v_{i}} y d L_{\underline{\lambda}}\left(y \mid v_{i}\right) & v_{i} \leq 0.5 \\ \int_{0}^{v_{i}} y d L_{\bar{\lambda}}\left(y \mid v_{i}\right)+\int_{0}^{0.5} y d\left(L_{\underline{\lambda}}(y \mid 0.5)-L_{\bar{\lambda}}(y \mid 0.5)\right) & v_{i}>0.5\end{cases}
$$

Consider type $v_{i} \leq 0.5$ deviating to a bid $b(z) .{ }^{23}$ Note that for $0.5>z>v_{i}$ :

$$
\begin{aligned}
\frac{\partial U_{\underline{\lambda}}\left(z \mid v_{i}\right)}{\partial z} & =G_{\underline{\lambda}}\left(z \mid v_{i}\right)\left[\frac{g_{\underline{\lambda}}\left(z \mid v_{i}\right)}{G_{\underline{\lambda}}\left(z \mid v_{i}\right)}\left(v_{i}-b(z)\right)-b^{\prime}(z)\right] \\
& <G_{\underline{\lambda}}\left(z \mid v_{i}\right)\left[\frac{g_{\underline{\lambda}}(z \mid z)}{G_{\underline{\lambda}}(z \mid z)}(z-b(z))-b^{\prime}(z)\right]=0
\end{aligned}
$$

The inequality follows because $z>v_{i}$, and when $\underline{\lambda}$ is close to zero, $\frac{g_{\lambda}\left(z \mid v_{i}\right)}{G_{\underline{\lambda}}\left(z \mid v_{i}\right)}$ and $\frac{g_{\underline{\lambda}}(z \mid z)}{G_{\underline{\lambda}}(z \mid z)}$ are close. ${ }^{24}$ A similar argument shows that for $z<v_{i}, \frac{\partial U_{\lambda}\left(z \mid v_{i}\right)}{\partial z}>0$.

When $z>0.5$, note that if $\bar{\lambda}$ and $\underline{\lambda}$ are close, then $\frac{\partial U_{\bar{\lambda}}\left(z \mid v_{i}\right)}{\partial z}$ and $\frac{\partial U_{\lambda}\left(z \mid v_{i}\right)}{\partial z}$ are close. Thus for some $\varepsilon>0$,

$$
\begin{aligned}
\frac{\partial U_{\underline{\lambda}}\left(z \mid v_{i}\right)}{\partial z} & <\frac{\partial U_{\bar{\lambda}}\left(z \mid v_{i}\right)}{\partial z}+\varepsilon \\
& =G_{\bar{\lambda}}\left(z \mid v_{i}\right)\left[\frac{g_{\bar{\lambda}}\left(z \mid v_{i}\right)}{G_{\bar{\lambda}}\left(z \mid v_{i}\right)}\left(v_{i}-b(z)\right)-b^{\prime}(z)\right]+\varepsilon \\
& <G_{\bar{\lambda}}\left(z \mid v_{i}\right)\left[\frac{g_{\bar{\lambda}}(z \mid z)}{G_{\bar{\lambda}}(z \mid z)}(z-b(z))-b^{\prime}(z)\right]=0
\end{aligned}
$$

where the last inequality follows because $z>v_{i}$, and since $\bar{\lambda}>0, \frac{g_{\bar{\lambda}}(z \mid z)}{G_{\bar{\lambda}}(z \mid z)}>\frac{g_{\bar{\lambda}}\left(z \mid v_{i}\right)}{G_{\bar{\lambda}}\left(z \mid v_{i}\right)}($ see footnote 24 ).

To see that bids are uniformly higher under ambiguity, note that in the case without ambiguity, the bid can be written as $b_{0}\left(v_{i}\right)=\int_{0}^{v_{i}} y d L_{0}\left(y \mid v_{i}\right)$. When $v_{i} \leq 0.5, \int_{y}^{v_{i}} \frac{g_{\lambda}(t \mid t)}{G_{\lambda}(t \mid t)} d t$ is decreasing in $\lambda$ since the integrand $\frac{g_{\lambda}(t \mid t)}{G_{\lambda}(t \mid t)}$ is decreasing in $\lambda .^{25}$ Thus, $L_{\underline{\lambda}}$ first order stochastically dominates $L_{0}$, implying that for $v_{i} \leq 0.5, \int_{0}^{v_{i}} y d L_{\underline{\lambda}}\left(y \mid v_{i}\right) \geq \int_{0}^{v_{i}} y d L_{0}\left(y \mid v_{i}\right)$. A similar argument implies that for $v_{i}>0.5, \int_{0.5}^{v_{i}} y d L_{\bar{\lambda}}\left(y \mid v_{i}\right) \geq \int_{0.5}^{v_{i}} y d L_{0}\left(y \mid v_{i}\right) .{ }^{26}$ Note

${ }^{23}$ The argument for $v>0.5$ is analogous.

${ }^{24}$ The derivative of $\frac{g_{\lambda}(z \mid v)}{G_{\lambda}(z \mid v)}$ with respect to $v$ is $\frac{2 \lambda z^{2}}{G_{\lambda}(z \mid v)^{2}}$.

${ }^{25}$ The derivative of $\frac{g_{\lambda}(t \mid t)}{G_{\lambda}(t \mid t)}$ with respect to $\lambda$ is $\frac{t^{2}(2 t-1)}{G_{\lambda}(t \mid t)^{2}}$.

${ }^{26}$ Integrating by parts and using the fact that for $v_{i}>y>0.5, L_{\lambda}\left(y \mid v_{i}\right)$ is decreasing in $\lambda$ :

$$
\begin{aligned}
& \int_{0.5}^{v_{i}} y d L_{\bar{\lambda}}\left(y \mid v_{i}\right)-\int_{0.5}^{v_{i}} y d L_{0}\left(y \mid v_{i}\right) \\
& =0.5\left(L_{0}\left(0.5 \mid v_{i}\right)-L_{\bar{\lambda}}\left(0.5 \mid v_{i}\right)\right)+\int_{0.5}^{v_{i}} L_{0}\left(y \mid v_{i}\right)-L_{\bar{\lambda}}\left(y \mid v_{i}\right) d y \geq 0 .
\end{aligned}
$$


that $\int_{0}^{0.5} y d L_{\bar{\lambda}}\left(y \mid v_{i}\right) \geq \int_{0}^{0.5} y d L_{\bar{\lambda}}(y \mid 0.5)$ and $\int_{0}^{0.5} y d L_{\underline{\lambda}}(y \mid 0.5) \geq \int_{0}^{0.5} y d L_{0}(y \mid 0.5)$, implying the result. 


\section{References}

[1] Bergemann, D., B. Brooks and S. Morris (2015), First Price Auctions with General Information Structures: Implications for Bidding and Revenue, mimeo.

[2] Bose, S., E. Ozdenoren, and A. Pape (2006), Optimal auctions with ambiguity, Theoretical Economics 1, 411-438.

[3] Bose, S. and Renou, L. (2014), Mechanism Design With Ambiguous Communication Devices. Econometrica, 82: 1853-1872.

[4] Chen, Y. P. Katušcák and E. Ozdenoren (2007). Sealed bid auctions with ambiguity: Theory and experiments. Journal of Economic Theory 136, 513-535.

[5] Church, K.W and P. Hanks (1991), Word association norms, mutual information, and lexicography. Comput. Linguist. 16 (1): 22-29.

[6] Crémer, J. and R. P. McLean (1988). Full Extraction of the Surplus in Bayesian and Dominant Strategy Auctions. Econometrica, 56: 1247-1258.

[7] Enke, B. and F. Zimmerman. (2013). Correlation neglect in belief formation. mimeo.

[8] Eyster, E. and G. Weizsäcker. (2011). Correlation Neglect in Financial DecisionMaking. Discussion Papers of DIW Berlin 1104.

[9] Gilboa, I. and D. Schmeidler (1989), Maxmin expected utility with non-unique prior, Journal of Mathematical Economics 18, 141-153.

[10] Glaeser, E. and Cass R. Sunstein (2009), Extremism and social learning. Journal of Legal Analysis, Volume 1(1), 262 - 324.

[11] Hanany, E., P. Klibanoff and S. Mukerji (2018), Incomplete Information Games with Ambiguity Averse Players, working paper.

[12] Kallir, I. and Sonsino, D. (2009). The Perception of Correlation in Investment Decisions. Southern Economic Journal 75 (4): 1045-66.

[13] Levy, G. and R. Razin (2018). Combining Forecasts in the Presence of Ambiguity over Correlation Structures

[14] Levy, G. and R. Razin. (2015a). Correlation neglect, voting behaviour and information aggregation. American Economic Review 105: 1634-1645. 
[15] Levy, G. and R. Razin. (2015b). Does polarisation of opinions lead to polarisation of platforms? The case of correlation neglect, Quarterly Journal of Political Science, Volume 10, Issue 3.

[16] Lo, K. C., (1998). Sealed bid auctions with uncertainty averse bidders. Economic Theory 12, 1-20.

[17] Ortoleva, P. and E. Snowberg. (2015). Overconfidence in political economy. American Economic Review, 105: 504-535.

[18] Renou, L. (2015). Rent Extraction and Uncertainty. mimeo.

[19] Salo, A. S., Weber, M., (1995). Ambiguity aversion in first-price sealed-bid auctions. Journal of Risk and Uncertainty 11, 123-137.

[20] Seidenfeld, T. and L. Wasserman (1993). Dilation for Sets of Probabilities. Annals of Statistics. 21 (1993), no. 3, 1139-1154.

[21] Wolitzky, A. (2016). Mechanism design with maxmin agents: Theory and an application to bilateral trade. Theoretical Economics 11: 971-1004. 